




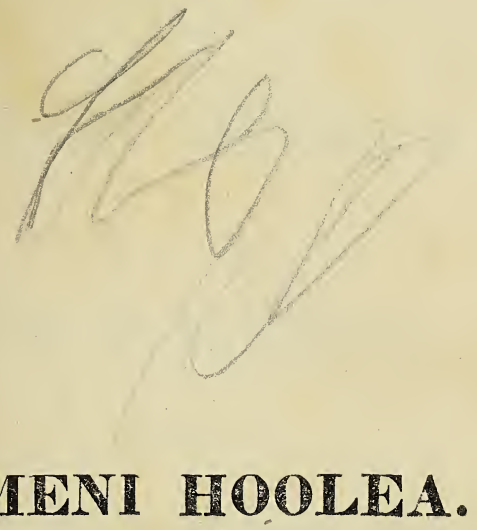

HIMENI HOOLEA. 


$$
\prod_{N A} \pi
$$

\title{
HIMENI HOOLEA.
}

\author{
HE IIAU
}

\section{MELE MA KA UHANE,}

E HOOLEA AI

Na kanaka, na keiki, na ohana, na elralesia,

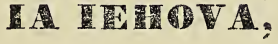

KE AKUA E OLA'I.

KA NA MISIONARI MEA PAI. 1847. 
is 


\section{HE OLELO HOAKAKA.}

He buke hou keia; ua huiia no nae na himeni kahiko me na himeni hou iloko; ua hoololi hou ia na himeni kahiko, a ua hooponoponoia na olelo hemahema. Ua oi aku paha ka maikai o keia buke mamua o na buke himeni hoolea mamua; lealea paha na haipule i ka himeni ana.

Aole keia o ka ke Akua maoli; ua hookumuia nae malaila; ua haku ia kekahi himeni mamuli okekahi halelu a Davida, a o kekahi hoi mamuli o na olelo hemolele e ae. Nolaila. o ke ano nui o na himeni, ua like pu me ka ke Akua. Ua unuhiia kekahi mau himeni mai ka olelo Berita- 
nia, a ua haku okoa ia kekahi ma ka olelo Hawaii.

Ua ku pono loa na himeni hoolea i ka poe haipule; mai ka wa kabiko loa mai, ua makemakeia na himeni hoolea i na kanaka o ke Akua. Hauoli maoli ka naau i ka heluhelu ana a me ka mele ana iloko o ka hale o Iehova, a ma kahi malu kekahi, a e pono i na haipule o Hawaii nei, ke waho loa i na mele kahilko o keia aina, e oki hoi i ka olioli ana, a e hapai ia Iehova ma na himeni hoolea me na leo ku pono. Pela kana kauoha, Kol. 3: 16. "E noho nui mai ka olelo a Kristo iloko o oukou, me ka naauao loa, e ao ana a e hoonaauao ana kekahi i kekahi i na halelu, a me na himeni, a me na mele 


\section{vii.}

ma ka Uhane, e oli ana i ka Haku, me ka maikai iloko o ko oukou naau."

$\mathrm{Ku}$ pono no hoi na himeni i la panaau; he mea ia e maemae ai ka naau, a e olioli ai hoi. He mea hoi ia e lana ai ka naauao $i$ ka wa mai, a pilikia, a luuluu, a mehameha paha. Pono hoi e ao aku i na keiki e panaau i na himeni, a e imi hoi $\mathrm{i}$ ke alkamai ma ka pa, ko, li, i pono ke mele ana. Oia ka mea e lilo ai na himeni, i mea maikai loa, o ka makaukau i ka leo.

E loaa no ia oukou ka himeni o kela ano, keia ano, ma ka nana ina hua nui maluna; penei, ina $\mathrm{i}$ makemakeia ka himeni no ke Akua, e nana i na hua maluna, No re AKr: A. Pela no na himeni no IESU, UHANE TEMOLEEE, HOOWELIWELI, a pela aku no. 



\section{HIINI。}

8.

E malana ia lehova me ka hauoli.

1 MA ko Iehova alo mau,

E moe hauoli ai oukou;

I ike e na aina a pau,

Oia ke Akua mana mat.

$2 \mathrm{Na}$ kona mana oịa mau, I hana mai ia kakou nei;

A hele hewa pu kakou,

Kii mai kela e hoola mai.

3 Nona na kanaka a pau, Na hipa nana i hanai;

Kaulana e ka inoa ou,

E ke Akua e ola'j.

4 Ma kou mau puka e komo ai,

Me ke aloha o makou;

Na ko ke ao e mele ae,

Hapai i kou inoa mau.

5 Nou ke aupuni o ke ao, Nou ke aloha nui mau; E ku paa mau ka olelo au, A pau loa aku keia ao. 
Ka mana o ke Akua.

1 KEI ka mana o Iehova,

I mau ai na mea a pau, Mai kinohi, a mau loa,

Ku paa oia, oia mau.

2 Aia hoí ma kona mana,

Kaa mau ka honua nei;

Ma ka poho lims ona

Na moana $i$ waiho ai.

3 O ka la, me la mahina, O na hoku, me ke ao, Ke kau nei ma kona lima, Malu maoli no lakou.

4 Liili no kela mau mea,

Nui e ka Taku mau, Aole pan i ka ikea,

Ko Lehova mana a pau.

5 Malu kana mau haipule,

Paa i kona lima akau, Aole loa e haule,

Hapai $\dot{i}$ kona mana mau.

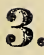

$$
\text { 8-7. }
$$

Te Akua maikai,

1 IIE Akua hemolele, Ire Akua no kakou; 
Maikai wale, hewa ole, Pono loa, ola mau.

2 Nani wale ko Iehova, Luli ole ka maikai;

Ahonui wale kela,

I ka hana pono mai.

3 Oia no ka Haku mana,

O na mea ilalo nei ;

Nana kakou nei i hana,

Aole na ka lima e.

4 Pono hoi e pule aku,

I ke ola no ke ao;

$I$ ola kanaka ia ia la,

I ola nui no kakou.<smiles>[AlH][AlH]</smiles>

Iehova ka Moi.

1 I MAI la ka Moi, Ka Haku o ke ao,

Maluna o na'lii,

$\mathrm{Na}$ haku hoi a pau;

Owau no nei,

Ke Akua mau,

Ke ola wau,

$\mathrm{E}$ pono ai.

2 Hoolohe pono mai, Oukou na'lii a pau, 
Ia kanawai maikai,

E malama oukou;

Na'u no i kau,

Ke kanawai,

I lohe ai,

Oukou a pau.

3 Mai kuhi mai oukou,

He Akua ole nei;

Owau ke Akua mau,

Aohe akua e;

E hilinai

Maluna o'u,

Ka Moi mau,

E malu ai.

4 E kanaka a pau,

Hoolono maikai mai

I kuu olelo mau,

Mai pale iki ae;

E huli mai,

Mamuli o'u,

E lelepau,

I pomaikai.

5 Na moku hoi a pau,

Na aina me na kai,

E nana mai oukou,

Paulele maoli mai; 
Iloko o'u,

E komo mai,

I loaa ai,

Ke ola mau.

Ko Iehova nani.

1 E KA makua e, he nani kou, He kupanaha kau mau hana a pau, Hoike na hoailona kini no, Ma ka honua nei, a ma ke ao.

2 I Ia la, hoku, mahina, me ke ao, Ua hai $i$ kou mana, me kou akamai, Ma na eheu o na hora a pau, Ua heluia'i kou lokomaikai mai.

3 Aka, ma kon manao hoola mai, I na enuhe kipi me makou, O kou huhu, o kou aloha nae, Ua hui pu maluna o Iesu-

4 Ano e maikai na nani o Iesu, I nani ai ko luna mau papu,

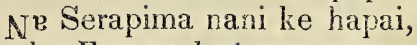
Iko Emanuela inoa ner.

5 E. ake au, e mele like pu, I kela mele ihi oia mau, E kuu mahalo nui no Iesu, E lea ai kuu leo i ke ao. 
$6-8$.

Ka pono o ko lehova kanawai.

I KE Akua o ke ao,

I lokomaikai mai,

Hoolaha mai la no kakou,

I kona kanawai.

$2 \mathrm{Na}$ i $a$ la i hai mai,

No ke aloha mau,

Na hua e pomaikai ai,

Na uhane o kakou.

3 E malama kakou,

I keia kanawai,

He pono, hemolele mau,

I kumu alakai.

4 E hele pu kakou, Ma keia pomaikai,

He alanui maikai mau,

He ala pololei.

5 E lelepau oukou, Ma keia pono mau,

I naauao, i pomaikai, .

I ola mau oukou.

r.

$6-8$.

Ke $\Lambda$ kua oiaio

$1 \mathrm{AUHE} A$ ka pono mau,

I pono ai kakou? Auhea ka pono e ola'i? 


\section{NO IKE AKUA.}

R imi pu kakou,

I ke Akua mau,

E pau na akua wahahee.

$2 \mathrm{Na}$ Iesu i hoopau, Koonei mau kii laau;

Pau pu na kapu o hakou:

1 keia wa maikas,

Lohea ke kanawai

O the Alua ola mac.

3 Eia ka pono mau, No ko ke ao a pau,

Ko lesu pono e ole'i:

E lelepau kakou,

Mamuli ona no,

Malaila mau e malu ai.

4 He alanui hor, E hele ai rakou,

A hiki loa i ke ao:

Hoomana! hoomaikai!

Iehova ka Moi,

Ke Akua peno oia mau.

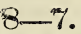

Fe aloha o ke Akua.

1 HE aloha ko Iehova,

I na kanaka a pau,

Hai mai oia i ke ola, 
Olioli no kakou:

Luaole ke aloha,

O Iehova ia kakou?

2 Aole no ko kakou pona,

Oia i aloha mai ;

Aole loaa mai ke ola,

Ma ko ia la kanawai:

Lawehala, hoomaloka,

Hewa wale, kakou nei.

3 Kana Keiki hanau kahi,

Ke 'Lii mana o ke ao,

I haalele $i$ kona wahi,

Kii mai oia ia kakou,

I pakele, ma o na la,

Kakou i ka make mau.

4 Kakou nei ka poe hewa,

$E$ imi i ka Haku mau;

Pule mau kakou ia ia la,

I pomaikai mau kakou;

I ola loa ia Iehova,

Ko ke ao nei poe a pau.

9.

8.

O Iehova ka Punhonua.

1 KE $A$ kua nona e ola'i,

A lele mai ka ino e,

Oia ke ku kokoke mai,

Me kana mau haipule nei. 


\section{NO KE AKUA.}

2 I naue ka honua nei,

Haalulu hoi na mauna $a$ pau,

Ke hoolein'ku i ke kai,

Aole o kakou makau.

3 Aia ka muliwai maikai,

No ko ke Lii kauhale mau,

Nolaila e pomaikai ai,

Malala nia me kakou.

1 Ka olelo an ka muliwai,

I. pono ko makou naau,

He mana e hauoli ai,

Na uhane luhi o makou.

5 Haunaele ko na aina c,

A kaua mai na hoomanu,

Ka leo ou ua pane mai,

Hehee tho la ko ke ao.

6 Iehora no ke Akua man,

F hapaiia e ko ke ao;

Me Ziona ia e noho pu,

Nona ko liakou ola mau.

10

8.

O lehova ke Fahnhipa.

1 IEKOVA no kuu IKahu mau, Kuu me' e pomaikai mau ai; No'u kela hoi, a nona wau,

Heaha ka'u e nele ai? 
2 Na ia la wau e alakai, Ma kona aina malu no, I kahe ai na wai maikai, I ola kuu uhane mau.

3 lauwana au i kahi e, Kii mai kela i ola au; A kai ma kana pololei, No kona inoa hoano mau.

4 Mawaena o na enemi, Ua hanai mai a maona wau, Ua piha a hu kuu apu nei; Kahinu pomaikai kuu poo.

5 Ka maikai me he aloha mau, E hahai mai i kuu mau la; Iini wale kun naau, Ii noho mau i kona pa.

6 A po kuiu la, a hele au, He awawa pouli make no; Aole loa wau makau, Malaila pu kuu Kahu mau.

11.

8. Ke Akua Kahi Kolu.

1 HOI.I aku kakou nei, Me ko kakou naau a pau, I ka makua no kakou, Ka Haku, ia Jehova mau. 


\section{NO KE AKUA.}

2 Iloolea aku hoi kakou

I ke Keiki maikai mau,

I iho mai ilalo nei

I ola e ola'i kakou.

$3 \mathrm{~K} a$ Uhane Hemolele hoi, Ke Akua ia e maikai ai, Ko kakou naau ino nei, I lilo i naau maikai.

4 Hoonani pono mau kakou,

I ka inoa o Lakou:

Ke Kahi-kolu nani mau, Hookahi Akua no kakou.

1 OLI aku ia Iehova,

Lanakilu! pomaikai!

He Alihikaua nani!

Pio e na enemi.

2 Parao me na koa ona,

Kana i hoolei $i$ ke kai, Nani no kou lima mana!

U'ola makou! pomaikai!

3 Kii mai nei lakou e luku,

Huhu wale ia makou-

Nou iho la kou makani,

Pauhia j ke kai lakou. 
4 Owai hoi kou mea like?

Hanohano wale kou,

Nani no kou hemolele,

Kupanaha, pono mat.

5 Llapai oukou ia Iehova,

Ke 'Lii $i$ lanakila ai;

Ia ia $e$ pio ai ka hewa,

Ola ia ia ka maikai.

13.

$$
\text { 8-6. }
$$

E hoolea ia Iehova.

1 HOOLEA aku ko ke ao,

I ke Akua mau;

Ua hana oia ia kakon,

A me na me' a pau.

2 E pule aku kakou nei,

I ka makua mau,

E kala mai i ka hala nei,

O ko kakou naau.

3 Kahea mau aku ia Iesu,

I ke Kamaiki mau,

E pipi mai ia kakou nei,

Me kona koko mau.

4 E noi aku hoi kakou,

I ka Uhane mau,

$\mathrm{E}$ hana maikai hou kela,

I ko kakou naau. 
E L hapai aku hoi oukou,

I kona inoa mau;

Iehova-oia wale no,

Ke Akua no kakou.

$$
3-7-4 .
$$

E hiilani aku ia Iehova.

1 PULE pono ia Iehova,

Hapai $i$ kona inoa mau;

Haku nani o ka lani,

Oia no ke Alrua mau;

E hoonani,

la lehova ola mau.

2 Nana no kakou i hana,

INana kakou i hanai,

Nana kakou i ao mai la,

Nona kakou e ola'i;

E hillani,

I ka Haku mana mata.

3 Ahonui ka Makua,

A haawi lilo mai,

Ke Kamaiki punahele,

I alana e ola'i;

Haleluia,

I ka Alana pono mau.

4 Tho mai kela ilalo,

No kakou i make ai,

Oia no ke Kalahala, 
I ko ai ke kanawai;

E hoolea,

I ke Kalahala mau.

5 Mai ka make, ala mai la,

Hoi aku la i ke ao;

A ia mau me ka Makua;

Ala no ka Uhane mau;

E hoomaikai,

I ka Moi o ke ao.

กร.

8.

Ko ke Aku ike.

1 E KE Akua mana mau, Ua ike oe ia makou,

Na hele me na hana a pau, Ke kino pu, me ka naau.

2 Ua ike e na maka ou,

E ke Akua ola mau,

$\mathrm{Na}$ hana me na olelo pu,

Maloko a mawaho no.

3 Maloko o ka lima ou,

Ke ala nei makou a pau,

Iloko, a iwaho no,

E malu ana keia ao.

4 Maluna ma ka lani no,

Malalo iho, ma ka po,

Malaila no kou lima mau,

E paa mai ana ia makou. 


\section{' NO KE AKUA.}

5) Nolaila, pono ia makou,

Ke noho me ka hoomanao,

Eia maanei na maka ou,

I hewa ole ai makou.

\section{8.}

E hoomaikai i ke Alkua.

1 ILUNA lilo o na ao, Malaila no ke Akua mau,

Ke 'Lii no oia o na 'lii,

Ko luna, ko ke ao Moi.

2 Auhea oukou na anela,

O ko ka lani poe kauwa, Hoike mai, a hoomaikai,

$\mathrm{Na}$ hana ana $\mathrm{i}$ hana'i.

3 Oukou no hoi, na hoahanau,

$\mathrm{Na}$ kanaka ma keia ao,

Hoike, a hillani no,

I kona lokomaikai mau.

4 Hoomaikai i ka Haku mau,

E ko ka lani, ko ke ao,

$\mathrm{Na}$ leo, hanu, na naau,

$\mathrm{E}$ hapai i na mele mau.

E hoonani i ke Akua

1 HOONANI, e hoonani mau,

I ke 'Lii maikai o na ao, 
He mana kona e paa ai, Ko luna, me ko lalo nei.

2 Hoonani a mahalo pu,

I kona lokomaikai mau,

Oia la ko kakou Alii,

$\mathrm{Na}$ ia la e hoomalu mai.

3 Nolaila, e na hoahanau,

Hoopau kakou i na makau,

Hauoli, a holoi ae,

I na waimaka kahe nei.

$4 \mathrm{Ka} \mathrm{Haku,} \mathrm{e} \mathrm{hoonui} \mathrm{ae,}$

I ko makou nei hilinai,

I na olelo kupaa au,

Nolaila mai ba pono mau.

5 Alaila, ina lilo e,

Ko luna me ko lalo nei,

Kupaa na uhane o makou,

Ma kou aloha oia mau.

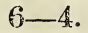

Ke Aku: akahi Kolu.

1 E KE 'Lii mana mau,

Kokua ia makou,

Na kauwa au;

Makua nani no,

A lanakila mau,

Hoomalu ia makou,

Makua mau. 
2 Ka Logou mana e,

Ka mohai e ola'i,

No keia ao;

Kokua pono mai,

I kau mau kauwa nei,

E kulou hoomaikai,

Imua ou.

3 Uhane mana mau,

Hoomaikai ia makou,

E launa pu;

E noho maoli no,

Iloko o makou,

Paipai, hoolianau hou,

1 na naau.

4 Ke Kahi-kolu mau,

Kokua ia makou,

E mele mau;

I ike ae makou,

Kou nani kamairan,

A pii a noho pu,

Ma kela ao.

19.

$$
6-8 .
$$

O ko lehova Hanohano.

1 IEHOVA ke 'Lii mau,

He hanohano kou,

Ua puni i ka nani mau;

He lei olino kou, 
He ahu ao haohao,

He ihiihi kamahao.

2 Ma kau olelo mau,

Ua onipaa ke ao,

Ka la, mahina, na hoku;

Kou noho-moi mau,

Maluna ma ke ao;

Mau kou aupuni, e Iesu.

3 Kupikio na kai,

A ala kue mai,

O ko ka po, o ko ke ao;

Hooweliweli mai,

lesu ke 'Lii maikai,

Malie mai, a malu mau.

4. He oiaio mau,

Na olelo au a pau,

Ma kou aloha e ku no,

Na kauwa pono ou,

Ma kou Ziona hou,

E mele $i$ kou aloha mau.

$$
6-4 \text {. }
$$

O lesu ka mea e ola'i.

1 UA hoounaia mai,

Ke Ola nani e,

Ke 'Lii maikai ;

No kona aloha mau,

I hanaka a pau, 


\section{Haalele i ke ao,}

\section{A iho mai.}

2 A $i$ hiki ole mai,

Ke Kalahala nei,

I mohai mau;

Ua make mau kakou,

Ka poe kiva no,

Malalo i ka po,

Poino mau.

3 'Hoilihune mai,

Ko luna Alii waiwai,

A make e;

I pomaikai bakou,

Ma kona hune no,

Maluna o ke ao,

E waiwai ai.

4 Na Iesu i lapaau,

$\mathrm{Na}$ mai o neia ao,

I ola mau;

Ua kuu mai no kakou,

I ka uhane mau,

I lilo ko ke ao

I naau hou.

5 Oukou na anela,

A me na kanaka,

E hapai pu; 
I ka Makua hoi,

Me Iesu ka Mlohai,

$\mathrm{K} a$ Uhane pomaikai,

Ke Akua mau.

2. Ko Iesu hoohaaha ana ia ia iho.

1 OWAI la i hoolohe mai,

I ka olelo no Iesu?

Owai la e paulele nei,

I ka Mesia maikai mau?

2 Ua iho mai, mai kela ao, Ke Lii pookela, pomaikai ;

Ka makamaka kamahao,

Ua noho malihini mai.

$3 \mathrm{Ua}$ ilihune ano e,

I waiwai nui ai kakou;

Kipaku pu ko lalo nei,

A me ko lalo o ka po.

4 Paopaoia'e la ke Lii mau,

No ko kakou mau hala no;

Maluna ona ka hahau,

I maluhia ai kakou.

5 Ua heluia me na aie,

A pule no ka poe bokai,

Nou no ka uku pololei,

$\mathrm{Na}$ aina au i kuai ai. 
1 E IESU ka maikai, .

Me e kou pono mau,

E ahonui mai,

E ao mai ia makou;

O oe no ke Kumu mau,

$\mathrm{E}$ pono ai ko keia ao.

2 Hoike pono mai,

I ko makou naau,

I kou mau kanawai,

I malama makou;

E Iesu e ha Haku mau,

E alakai i ko ke ao.

3 Hoonaauao mai hoi,

I kanaka a pau,

I maopopo ai,

Ke ola no lakou;

E Iesu ko ka pono La,

I ike ai na maka paa.

4 Nau, e ka La maikai

E wehe mai ke ao,

E pii iluna ae,

I ao na aina a pau;

I nui mai ka naauao,

Ka pomaikai o keia ao. 
6)

$8-6$.

E mele hoomaikai ia Iesu.

1 KA poe nani o ke ao,

Na anela maikai,

E mele pomaikai lakou,

I ke 'Lii e ola'i.

2 Oukou na kanaka a pau,

A Iesu i kuai,

Hauoli pu, a hapai mau,

I ko ke ao mohai.

3 Ua eha oia no kakou,

I ola ko ke ao;

E ola kakou nona no,

Malaila, lelepau.

4 He mohai ola mana nei,

He kalahala mau,

E mele, a e hoomaikai,

Ko keia ao a pau.

24.

8.

O Jesu ka inoa maikai.

1 O IESU ka inoa maikai,

He hale kiai mana mau,

He inoa e pomaikai ai,

$\mathrm{N}^{\mathrm{e}}$ lahuiaina a pau,

He kupaianaha no nae,

He nani, he ihi, he hao,

Aohe inoa e ae,

E ole mau ai ko ke ao. 


\section{NO IESU.}

2 Ke kaula, kahuna, moi,

Ke Ola Akua mau no,

Ma kona inoa maikar,

E pelu na kuli a pau;

Ka mua, ka hope, Iesu,

I make, a ola mau no,

I malu mai ai ko ke ao,

I pio mau ai ko ka po.

3 O Iesu ka inoa maikai,

E kaena ai ka naau,

Ko kakou Akua no nei,

E paulele loa kakou;

Kaulana olelo uwao,

Mesia, alii, alakai;

E hapai mau ia i ke ao,

Ko lesu inoa maikai.

8.

Ko Iesu aloha ia kakou.

1 UA alohaia mai kakou,

Ka poe kina wale no;

Iehova ke aloha mai,

I ola ia ia kakou nei.

2 Ua aloha Iesu ia kakou,

A tho mai i neia ao;

I kahe kona koko mau,

I mea holoi ia kakou.

3 No ko Iesu aloha mai,

Ua make ia no kakou nei 
O kona make ana no,

Ka mea e ola ai kakou.

4 E Iesu, e ka Haku mau, Holoi mai oe ia makou;

Ninini mai $i$ ka Uhane ou,

I naau hou, i ola mau.

1 E KUHIKUHI mai,

I ala no makou,

Nau hoi e alakai,

Ma ka aoao pono ou;

E Iesu e, ka alana mau,

E hiki ai i kahi ao.

$2 \mathrm{E}$ hooikaika mai,

I ko makou naau,

I lohe pono ai,

I ka olelo mau;

Nau, e Iesu, ka olelo mau,

E maemae ai ko keia ao.

3 E hoolaia mai,

Na uhane o makou;

Ua make i ka mai,

Mai make maoli no;

E Iesu e, ke ola mau,

Kokua mai, o pau makou. 
$8-7$.

Ke ola nui.

1 I KUU pilikia ana,

A mai make i ka po,

Na kuu Haku i hoolana,

Aole ae la i poho;

E hauoli!

Haleluia!

I ke ola nui nei.

2 Na Iesu i kala wale,

Kuu mau hala, kuu aie,

Ma ke koko hala ole,

Ola hou na make nei;

Na mohole,

Haleluia,

I ke ola nui nei.

3 Hele ia ia, manaoio,

E na lawehala a pau;

Hoolaa lilo pu na kino,

'Na uhane, na naau;

Mele nui,

Haleluia,

I ke ola nui nei.

4 Pomaikai mau na haumana,

Lanakila.i ke ao,

I ko Iesu kuu mau ana,

I ke ola oia mau; 


\author{
E ko luna, \\ Ifaleluia, \\ I ke ola nui nei.
}

28.

8.

Ke Kahuna e ola'i.

1 O IESU Kristo ke 'Lii mau,

he Akua nui o kakou;

Ko kakou Haku mana nei,

O Tesu ka Moi maikai.

2 He ola ja no kakou nei,

He nui kona aloha mai ;

No kakon ia i make ai,

Ka mohai nui e ola'i.

3 I koho nui ae kakou,

Ia Iesu i kahuna mau,

I pau ka eha, hui, mai,

Kahuna mana e ola'i.

4 He ola nui o Iesu,

Na ka. Wakua i kuu mai,

He pono e mahalo pu,

Ia ola mau e pono ai.

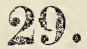

8.

O Iesu ka Pailota

$1 \mathrm{KE}$ holo nui nei kakou,

I nei moana o ke ao;

Kupikipikio ke kai ;

O lesu nae ke Alakai. 


\section{TO $\mathrm{N}$ SU.}

2 He uwila, he hekili no, He ino nou ikaika mai, Aole makou i pohn, O lesu no ke Alahai.

3 Ina popoi nui hou, Na ale o la poe huhu; Eia ka mea e lana ai, O Iesu mau ke Alakai.

E hoomanao mai ia'u.

$1 \mathrm{HE}$ ahonui wale mai, Iesu ka Haku mau, E imi au i hou maikai, E hoomano mai ia'v.

2 E Iesu e, e nana mai, Ua hewa loa wau; Pehea la e pono ai ? E hoomanao mai ia'u.

30 oe no kuu alakai, Ma keia ao a pau, $O$ oe no ke ao mau mai; $\mathrm{E}$ hoomanao mai ia'u.

4 A pau kuu wa è ola nei, A make aku wau;

E lokomailiai nui, e, $\mathrm{E}$ hoomanao mai ia'u. 
5 A komo oe, e kuu Moi, I kou aupuni mau, A hoopaiia ou enemiE hoomanao mai ia'u.

31. $\%$. Ke aloha e ola'i.

1 EIA mai ke mele hou, O Iesu ka Mohai mau; Nona ke aloha mai, Ke aloha e ola'i.

2 Pilikia pu kakou, Mai poino i ka po; Pomaikai, ua loaa mai, Ke aloha e ola'i.

3 Noho pio luhi no, Kii mai Iesu ia kakou; Kuai no a wehe mai, Ke aloha e ola'i!

4 Ke aloha mana nei, Malu hou na enemi; Isanakila nani e, Ke aloha e ola'i.

$5 \mathrm{Na}$ haipule ma ke ao, Mele hou, a mele mau; Oia mau kuu mele nei, Le aloha e ola'i. 
1 ALOHA nui ko Iesu,

I kona kini ponoi ;

A lokomaikai wale mai,

I kona poe enemi.

2 Pepehi ae na hoomaau,

I ka Mesia maikai mau,

A lilo oia i mohai,

No kela mau powa hokai.

3 Aloha kana pule e,

E kala mai ia lakou nei,

A ala oia, imi hou,

I ola nui no lakou.

4 A pii pomaikai i ke ao, He lokomaikai oia mau;

A kuu hou mai i Kumu hou,

I pomaikai mau ai kakou.

5 Auhea la ke aloha e, Me ke aloha nui nei?

Nou, e Iesu, kakou a pau,

Nou loa ko kakou naau. E na haipule launa pu; 
Ka oukou mea e mele ai,

Ka lokomaikai nui nei.

2 Popilikia no oukou, Aloha maoli mai lesu; Hoola kupanaha mai, Ka lokomaikai nani nei.

3 Ka poe ku e, la poe kina, E huli mai i keia la ; He Ola lesu no kakou, Te lokomaikai kamahao!

4 Ka poe wacia e Iesu, Aole e poino hou; O kona aloha ia lakou, He lokomaikai oia mau.

5 Ko keia ao, ko kela ao, Ku nui mai e mele pu, He nui, nani, oia mau, Ka lokomaikai o Iesu.

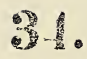

8.

Ka Puahonun majkai.

1 HE kulanahale ku paa, No kakou e pomaikai ai, Ka pono o lesu ka pa, He punhonua maikai; E wehe ia puka oukou, I komo ka poe maikai, 


\section{NO IESU.}

I ola mau loa lakou,

Ke paa ma ka mea pololei.

20 oe ke malama mai,

I malu mau loa lakou, Ke lelepau ma ka maikai,

Ma ou la ko lakou manao:

E paulele loa oukou,

I ko ke ao Haku maikai,

Iehova ke 'Lii oia mau,

He mana mau no e ola'i.

3 E ala, na haipule nei,

E himeni nui oukou,

E komo ia wahi maikai,

A hala na ino a pau,

Ke kali ae nei no makou,

1 malu i kou kanawai-

E hauoli mau ko ke ao,

O Iesu ke 'Lii e ola'i.

5

$\%$

"Eia no ke aloha,"

1 MELE pono ia Iesu,

Hoomaikai ia mohai mau;

Oli $i$ kona aloha mai,

Ke aloha e ola'i.

2 Na ke Akua maikai mau,

I aloha mai ke ao,

Nana i haawi mai,

Kana Keiki e ola'i. 
3 E na pio i ka po, Kii mai lesu ia oukou; Nana oukou i kuai, Me ke koko e ola'i.

4 Olioli mau oukou, Ke paulele ia Iesu; Pomaikai ua loaa mai, Ke aloha e ola'i.

5 E ka Iesu huakai, I ka lani $e$ hele ai, Hele me ka hoomaikai, Ke aloha e ola'i.

6 A i pomaikai kakon, Halawai hou i ke ao, Ke aloha e ola'i, Oia mau e mele ai.

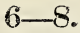

Ka nani o ka Mesia.

1 He hanohano mau, Ko luna Alii maikai; Mahalo loa kuu naau, I kona nani e.

2 E ola pomaikai, E ka Mesia mau;

He maikai mau kou nohodli, Aole loa e pau. 
3 He mana, he maikai,

$$
\text { Kou lima, e Iesu; }
$$

Hoolilo $i$ ou mau enemi,

I mau ohua nou.

$4 \mathrm{Ka}$ pono kou manao, Kou makemake mau;

Inaina loa kou naau,

Na hana hewa a pau.

5 Ka olelo pololei,

Ka pahikaua au,

Ka mea e lanakila ai,

I malu ai ke ao.

6 E huli koke mai,

Ko keia ao a pau,

Hanoli i ka pomaikai,

O kona aupuni mau.

37.

$$
8-7 .
$$

Ko fesu make ana ma Kalevari.

1 MAI ka lani, iho mai la,

O Iesu e imi mai,

I na uhane hele hewa,

Ia ia lakou e ola'i;

Lokomaikai wale kela,

Lohe ole ko ke ao,

Ukiuki wale ia ia,

Kue i ke Ola mau. 
2. Aia ma Ierusalema, Ku na lunakanawai, Hoahewa hewa ae la, I ka Moi akahai ; Makia i na lima ona, Me na wawae i ka hao; Kahe kona koko mana, No ka hala o ke ao.

3 Kaulia oia ma ke kea, E na kipi hoomaau, Nana lakou i aloha, Imi ola no lakou ; Pule i $a$, "E kuu Makua,

E aloha ia lakou, Kala i ko lakou hewa, No ka ike ole no."

4 Pouli wale ko ka lewa,

Olai ka honua a pau, Pau aku la, wahi ana, Kulou iho kona poo; Make e! aloha ino!

Kei ke aloha ia kakou! Ena hewa kona kino! Kei ke Kalahala mau!

Ko lesu apu awaawa.

1 AUWE, e kuu $\Lambda$ kua e !

Pehea la kau haalele mai? 


\section{NO IESU.}

Kahea no au a po ke ao, Aole ou kokua ia'u ?

2 Ina he mea hiki nei, E lawe oe ia apu e, He hemolele maoli kou, E ko ka makemake o'u.

3 Walana mai la lakou ia'u, Hoohewa mai a hoomaau, Huhu na mea hihiu hae, O mai $i$ kuu lima, kuu wawae.

4 Auwe! e kuu Makua e!

E kala mai ia lakou nei, Aole lakou ike mai,

I kau Mohai e malu ai.

5 Aloha wale! e Iesu, Me e kou eha ia makou;

Ia oe ko makou naau, E lílo i ohua nou.

1 KE ola! o ke ola mau! Hauoli pu kakou! Ua pale ko kakou makau, Na eha hoi, ua pau.

2 Maloko o ka malu po, Kakou i moe ai ; 
Ua ala hou nae ia Iesu, He ao, he pomaikai.

3 Ke ols- e hookaniia'e,

A puni keia ao;

Koluna e hookani mai,

Ia ola, oia mau.

40

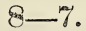

IKe ala hou ana o Iess.

I AHONUI wale Tesu,

Eha hewa no kakou,

tho i ka malu make,

Ola lanakila hou.

2 Hemo ae la ka pohaku,

I k $a$ elele o ke ao, Ala wale mai ka llaku,

Lanakila mana mau.

3 A kauoha mai, " $\mathbb{E}$ hele, $\mathrm{E}$ ao ae $i$ na aina a pau,

Ola no ka poe paulele-

Lohe ole-make mau."

4 Iloomaikai i na haumana,

Hoi iluna i ke ao,

Na lakou i hookaulana,

Ka Iesu olelo mau.

5 Ola oia i ka lani,

A kakali ia kakon, 


\section{NO IESU.}

Oli mau oukou iluna, Muli, e na aina a pau.

6 Kali me ke kiai aku,

Huli io, pule mau, Aia hoi mai ka Haku, Pii pu kakou i ke a.

4

$$
8-\%
$$

Ke aloha hoopakele.

1 E HOOMAKA i ke kani, Mele maikai ia Iesu;

Ia ia oukou e hiilani,

Nona no ka nani mau.

2 E na mea hoomaopopo, I ke ola o ke ao;

Oli no kolaila pono, No oukou i makaukau.

3 Oukou hoi ke mihi hala, Hoomohala $i$ ka makau;

Na lesu oukou e kala,

E hauoli ka naau.

4 Oukou hoi na mea auwana,

Mai ka pa nei o Iesu,

Huli koke ma ka Alana,

I pakele ai oukou.

5 Oia no ke koa mana,

I hee ai na enemi, 
46

\section{H I M F N I.}

I pau ai na kipi ana, Ma na aina o ke Alii.

6 Ho mai oukou i alana, E kaumaha ia lesu; Pela kakou e hoomana,

A kaa hope keia ao.

4.20

$$
8-7 .
$$

Ka eha ano e o ke Kahuna nui

1 KO makou Kahuna mana,

Ko makou aloha nei,

Ma ka inala, kulou ana,

No makou i luuluu ai ;

Me na kulu koko nui,

Helelei na kulu hou,

Kei ka ulono walania!

A wahi a ka apu ou.

2 Eha nui no kou kino, Ma ke ke $\alpha$ i pepe ai, I pau ai ko makou ino,

I ko ai ke kanawai;

Pau aku la-e paulele,

Aole kuihe oukou ;

Kei ka mohai hemolele!

Ma ia kea ke ola mau.

3 Mano kona pilikia-

E aloha mau kakou,

Mihi me ka walaniag 
Auhea kona like ana, Fai oluna a lalo nei ?

Ma ona la e hoolana,

E aloha ia mohai.

4 臬.

$$
\text { Pauka Levi Oihana. }
$$

1 AIA hoi! ko lesu leo,

Wai ke kea i pae mai;

Lohe mai e ko ka lani,

Lohe pu ko lalo nei;

"PAU AKU ra!"

Leo lanakila nae!

2 "Pau aku la," leo nani!

Ko ke ao nei pomaikai!

Kei ka pono o ka lani,

No kakou i loaa mai;

Hemolele!

Ko ke ao mea e ola'i.

3 Pau ka Levi oihana;

Pau kolaila mau mohai;

Pau na kanawai hailona,

He aka no ka mea maikai;

Ko aku la,

I ka Mohai maoli nei.

4 Pau ka kakou aie nui,

Paa ke kanawai maikai,

Рau ka mana o ka make, 


\section{NO IESU.}

Pau ka hana e ola'i;

Pau ia Iesu;

Ahonui! akahai!

5 E ko luna, me ko lalo,

Hapai $i$ ko ke ao Mohai;

Nani ka Iesu mau hana!

Pan ia i ka pololei ;

Haleluia!

O Emanuela nei.

15.

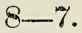

$O$ ke ola hou ana.

1 AIA ko ke ao nei Ola,

Pio no i ko ka po;

Eha nui, make wale,

Nalo no, auwe ke ao.

$2 \mathrm{Na}$ ko luna, ka pohaku,

Olokaa a hemo ae-

E ka make, kuu hou aku,

I kou pio nui nei.

3 Aia ka! ke ola io!

Hee aku la ko ka po;

U'ola hou mai nei ke Pio,

Ola lanakila mau.

4 Ko makou Alihikaua,

Kumu o ke ola hou,

Pii $i$ kou noho hanohano,

Nou no hoi ke aupuni mar. 
5 Pono nou e hookunaina,

I kou mea i pio ai;

Lawe hou i kou mau aina,

Nau no e hoomalu mai.

6 Aia pio kou ohua,

A i nalo pu makou,

Nau e wehe mai ka ilina,

I ala hou a ola man.

48

$\%$

Ko Iesu pii ana $\mathrm{i}$ ka lani.

1 PONO mau ka la maikai,

A lesu i pui hou ai ;

Noho hune me kakou,

Noi iluna i ke ao.

2 E na anela maikai, The $i$ kona pii ana'e;

Wehe, e ná puka mau,

Komo ke 'Lii o ke ao.

3 Lanakila mana mau,

Ola loa, i ke ao;

Nui kou aloha mai,

Nani wale kou maikai.

4 Kuu mai i ka Uhane ou,

I pomaikai mau makou;

Hoomaikai, a makaukau,

Pii pu makou i ke ao. 


\section{NO IESU.}

4 圔?

\%-6. 6.

Ka Nesia ma Ziona.

1 No Iesu ka lionua,

Me kona mau waiwai;

Na ia la i hookumu,

Maluna o ke kai;

Owai la ka hanauna,

O keia ao a pau,

E pii i kona mauna, Ka puu hoano mau?

2 Ka lima hewa ole,

A maemae ka naau,

He kiekie ole,

He oiaio no;

He hoomaikai mai nona,

Mai o Iehova mai,

A loaa mai ka pono,

E pomaikai mau ai.

3 Oia la ka hanama,

I imi ia lesu,

A pii ia i Ziona,

Ka mauna ihi no;

E ku no ma ke alo,

O ko Iakoba Aii,

Malaila e mahalo,

I ke 'Lii nani e. 
4 E weheia na puka, Hapai na pani mau,

A e komo ae iloko, Ka Moi nani no;

Owai ka Moi nani?

Ka Haku mana mau;

Iehova Sabaota,

Ka Moi o ke ao.

48.

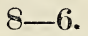

Ka hoalii ana i ka Mesia.

1 HOANO ko Ziona puu,

Malaila makaukau,

Ka noho moi no Iesu,

Ke Lii hoano mau.

2 Aloha wale, e Iesu!

E moe mai na 'lii!

$\mathrm{Na}$ anela e kulou pu!

Emanuela nei!

3 Na kauwa pio, luhi e!

Hoolana na naau,

E poni ia Mesia nei,

I Moi no ke ao.

4 Lulumi mai, e moe pu,

Ko keia ao a pau,

$\mathrm{E}$ hoalii hoi ia Iesu,

Ke Lii Pookela mau.

5 E na haipule pomaikai,

I komo i ke $\mathrm{ao}_{2}$ 


\section{NO IESU.}

E kau ae i ka lei Moi, Hoonani $i$ ke Lii mau.

6 Hosana! ola! e Iesu!

Ko makou Haku mau,

E kau ka lei maluna ou,

Pomaikai ko ke ao!

49.

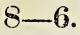

fiosana ia lesu.

1 E KO ke ao, hauoli pu,

E huli koke mai,

Hooiaio ia Iesu,

I Haku e ola'i.

2 Oukou ka poe i hanau hou,

Na kona Uhane mau,

Ua pale ae ko oukou po,

Hoomana naauao.

3 Kakou no hoi, e hoomaikai,

Hosana ia Iesu,

Eia'e ke Lii, ke hele mai,

Ka Poni nani mau.

4 I noho moi, nona no,

Ma ko kakou naau;

I lei maikai no kona poo,

O ka mahalo mau.

5 E pomaikai na aina a par, Ke lilo ia Iesu; 
E malu maoli keia ao, A malu oia mau.

50.

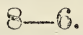

Ka houlii ana in Iesu.

1 HOOKANI i ke 'Lii maikai

E moe na anela;

E lawe mai $\hat{\imath}$ ka lei alii, No lesu ke 'Lii mau.

2 Oukou na kanaka maikai,

Maluna o ke ao;

No Iesu onkou i ola'i,

E hapai $i$ ke 'Lii mau

3 Oukou i noho naaupo,

A puka mai ke ao;

Ho mai i lei hoano no, Ia lesu ke 'Lii mau.

4 Oukou ka poe $i$ hanau hou, Hoawi na naau;

I lei alii no hona poo,

No Iesu ke 'Lii mau.

5 Na kanaka mai o a o,

$\mathrm{Na}$ aina hoi a pau;

E hoalii mau ia ia no, E hapai $i$ ke 'Lii mau.

6 Kakou no hoi e ola nei,

E oli me lakou; 


\section{NO IESU.}

E kau i lei alii maikai,

Ia Iesu ke 'Lii mau.

$6-8$.

He mahalo i ka Mesia hanohano.

1 HE 'Lii hoano mau,

Ke 'Lii e malu ai;

Hauoli pu e ko ke ao,

I ka Mesia nei.

2 Kakoo, e ka Min,

I pahiliaua nau;

E en e lanakila mai,

A malu keia ao.

3 He kookso pololei,

Kou kookoo moi mau;

Kou noho moi, he maikai,

E mau ke aupuni ou.

4 Na kou Akua no,

$O$ oe i poni mai,

I moi, i pookela mau,

Maluna o na lii.

5 E eu! a pomaikai, Hoomalu i ke ao;

E hou i kou mau enemi,

I. malu pu lakou

6 Hoolohe pu oukou,

Mahalo ia Iesu; 


\section{H I M E I.}

Ke 'Lii Mesia pomaikai,

He hanohano mau.

52.

$$
\begin{gathered}
6-4 . \\
\text { Pono Iesu. }
\end{gathered}
$$

1 E na haipule nei,

Hoolaha wale ae,

Mai o a o;

Ke aloha o Iesu,

I kanaka a pau,

A hookaulana mau,

Pono Iesu.

2 Mohala na makau,

E pau na kanikau,

Hauoli mau;

Hoonani i ke Lii,

E ala a kani ae,

$\mathrm{Na}$ leo mele nei,

Pono Tesu.

3 O na puali mau,

Na hoa o Iesu,

Ma kela ao;

- Ke mililani mau,

Na lira a lakou,

$\mathrm{Na}$ kumebala pu,

Pono Iesu.

4 A hui pu kakou, Ma ko ka lani puu

E lawe pu; 


\section{NO IESU.}

Na lira kamahan,

Na kumebala mau,

A mele mau kakou,

"Pono Iesu."

1. E mele oli, e, Ka leo himeni.

Leo maikai;

Ko luna poe maiksai,

A me kolalo nei,

Hoolea nani e,

Iesu ke 'Lii.

2 E hookaulana ac,

I kona nani e,

Mai o a 0 ;

Ka lani me ka po,

Ko keia ao a pau,

Hiilani ia Iesu,

Ka Haku mau.

3 Ua lanakila mau,

Maluna o ka po,

Me keia ao;

Va make, a ala hore,

$\mathrm{Uia}_{\mathrm{a}}$ pii ae $\mathrm{i}$ ke ao,

Ko keia ao nwao,

Hauoli mase.

8 
4 Ke kali nei kakou,

I kona iho hou,

Nai luna mai;

Me kona poe a pau,

I pau ko keia ao,

A lanakila mau,

lesu ke Lii.

\section{Thane Hemolele.}
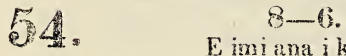

E imiana $i k a$ Uhane.

1 UHANE man hoolono mai, He ahonni kou;

Ke noi aku nei makou,

I kou aloha mau.

2 Uhane Alrua mana $e_{3}$ 'Aloha ia makou;

E iho mai ilalo nei,

Nou mai ka naau hou.

3 Uhrne maikai, hele mai,

Ke kali nei makou, F biki kolie mai ia nei,

I pono ai makou.

4 If lama hoi, e noho mai, Ha ko mahou naan;

Hoolei a ka ino nui nei,

li kint hoi i pau. 
5 Uhane Hemolele mau,

E lokomaikai mai;

E noho loa me makou,

Nou makou e ola'i.

5.

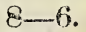

E kokua mai ka Uhane.

1 E IHO, me ka mana ou, Ka Uhane maikai mau,

Hoa i ke aloha hou,

Ma ko makou naau.

2 Nana i ka hihia nei,

Ka hei i keia ao!

Pehea makou e mele nou,

E kii i ola mau?

3 A ku makou e mele ae,

Nanea na naau,

Hoolea na leo o makou,

Auwana ka manao.

4 E mau anei, e Iesu e, Ia hewa o makou?

E manle ke aloha nei?

He nui wale kou!

5 E iho, e ka Uhane mat, Kau mai ka mana ou; Kuu mai ia aloha o Iesu,

A nui ko makou. 
56.

8.

He pule no ka Uhane.

1. Ma ko Iesu inoa maikai, Kulou makou, Iehova e, A moe ma kou mau kapuwai, E mohai i ka pule nei.

9 Ho mai i kou Uhane mau, I keia aha halawai; lini ko makou naau, E iho launa koke mai.

3 Anoi makou e lohe hou, I kona leo mana mau, Ke pane mai ka Uhane ou, Hauoli ko makou naau.

4 E alakai $i$ au mau kauwa, E mihi, pule, hoomaikai, L launa $i$ kou Uhane las, A me ia mau e hele ai.

5 E hana nui i keia la, Hoohanau hou i na hokak A huli nui na aia, 1 hau Mesia e ola'i.

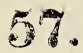

$$
6-8 .
$$

Ke Kumu àamai.

1 KO makou Haku ma, Ko makon Alakai, 


\section{UHANE HEMOLELE.}

Ke Kumu mau na ko ke ao, Ke Kumu akamai.

2 E ao mai ia makou,

Ka poe naaupo,

Aloha $i$ au haumana nei,

Ho mai i naaua.

3 Hooponopono mai

I ko makou naau,

I komo kau kauoha nei,

I ano hou makou.

4 Ko kanawai maikai,

Ko olelo pololei,

E hoopaa loa i keia la,

I paa ia makou nei.

8.

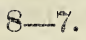

Ni mana o ka Uhane.

1 E K $A$ Uhane hemolele,

Me kou mana, e iho mai ;

Hooauhee i ka pouk,

Mai o makou aku nei:

Hoakaka i kau pono;

Pale i ka naaupo;

Kinai i ko makou hewa,

$f$ ole ai e ulu hou.

$2 E$ ao mại ia makou e mihị,

Hana hou i na naau, 
E kukuni $i$ ke aloha, I aloha mau makou; Hooikaika mau mai oe,

I ko makou nei manao, Hoala hou i ka paulele Ia Iesu, ka Mohai mau.

3 I k ${ }^{2}$ Uhane Hemolele, E alakai mau ia makou:

Hoauau i na whane, I ke koko o Iesu: Nau makou e hoohauoli;

Hoonaemae i na naut ; Me makou e noho loa; Lawe lilo i ke ao.

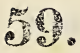

$$
\text { 6--8. }
$$

He pale i ka Uhane.

1 UIIANE Laa, kuu mai,

I kou kukuna mau,

E pale i ha pouli nei, O ko makou naau.

2 Nau e hoike mai,

Na hewa o makou,

Es alakai ma ka mohai, Ke koko o Iesu.

3 Nau e hoala mai

Ke aloha o makou, 
I ala hou ka hilinai

Ia lesu, ke 'Lii mau.

4 Nan e holoi mai

I ko makou naau;

I maemae, i ikaika ae

Ko makou nei manao.

5 E noho launa mai

I ko makou naau,

A na makou e hoomaikai

Ke Kahi-kolu mau.

8.

He pule i ka Utiane e hooluolu mai.

$1 \mathrm{~K} A$ Uhane mau, hoopuka mai

I na kuhma nani ou,

I pau na mea e luhi ai,

1 pau ka pouli o maliou.

2 Hoomaopspo ia makou, I ko ka naau hewa noi, F kai ia makou io Iesu, Ma kona koko e ola'i.

3 Nau no na hana mana nei,

I maemae hou ai na naau,

I ola hou, i pololei,

Na uhane hei i keia ao.

4 Hoolana hou i ka manao,

Ke kanalua e hoopau; 
Hoonui iloko o makou,

I ke aloha ulu mau.

5 Hoolilo io ia makou I luakini pono now; A mele nui na naau I kou aloha oia mau.

61. 8-6.

Kat ua ana oka Uhane.

1 KA ua i haule mai,

Ma koonei kihapai,

Ka mea ia e ulu ai,

Na mea hanu ne:.

2 Ka ua hoi e ulu ai, Ka olelo a Iesu,

Ka thane i $a$ mailuna mai,

Kia Uhane mana mau.

3 Ka Chane e, e iho mai,

Ma keia kihapai,

Hookupu i na hua maikai,

I luluia mai.

4 I malu ko makou naau,

Ma kou mau kanawai,

I hua koke ae makou,

He nui, a maikai.

¿ Pela no hoi e ulu ai,

Ka olelo maikai au, 


\section{HOOWELIWELI.}

\section{A huli mai ka poe kue, He lehulehu no.}

\section{Hooweliweli.}

8.

E poino ka poe hewa

1 Aloha ino o lakou,

Na kanaka i hoomalau;

Ko lakou ola e ola'i,

Ka lakou e hoino nei.

2 He naaupo hoi to lakou, He paakiki, he kuli mau,

O na uhane makamac,

Ka lakou e pepehi ai.

3 Walea maoli no lakou,

I na aoao o neia ao, Aka, pokole wale no,

Ka lealea o lakou.

4 He hope weliweli no,

A pau ko lakou ola nei;

E iho lakou i ka po,

Malaila mau e make ai.

5 E Iesu, e kokua mai, I na uhane o makou; E hoopakele waik ae, I $a$ hope ino o lakou. $3^{\text {* }}$ 
63.

$6-8$.

Ka hope o ka poe hewa.

1. HE $A$ kua pono no, Maikai ke malama ae;

Hookano nae na hoomaluu,

A u ka poe maikai.

2. A waiwai na hokai, Hua no kuu naau; Hookiekie na haakei, A hanohano no.

¿ Pehea la kuu manao?

He pono ole no,

A komo au i kou Heiau,

Ikea ka pono ou.

4 Ma kau olelo no,

Hoomaopopo wau.

Maanei, he ola ko lakou,

Ma o he make mau.

- Ile pali ku pahee,

E hele ai lakou:

Auwe ka lua e pau ai :

Ka lua ahi mau!

6. Ke kulou nei no wau

Imua ou, ke Lii ;

Kuu waiwai nei ke $A$ kua mu,

Nou wau, nou ponei. 


\section{HOOWELIWELI.}

$61.6--8$.

E viki mai ka mea makewai.

1 OWAI ka makewai,

I ane maule no?

Eia ke kumuwai maikai,

Ke koko o Iesu.

2 He wai ia e ola'i,

No luna no ke ao;

Ka poe $i$ inu ia wai,

Ua ola mau lakou.

3 Auhea na makewai,

E wiki mai oukou ;

E lawe no ia wai maikai,

He wai hoola mau.

4 Kii mai e inu no,

Kena ka makewai;

E lawe nui no oukou, He wai makana nei.

5 I ono wai kakou,

Manao ia kumuwai ; Ko Iesu kolso mana mau,

Akahi wai maikai.
65.
$8-7$.
O lesu ke kahu e maha'i.
1 "HELE mai ka poe luhi,
Na luuluu e hahai mai, 
Owar ke Kumu ahonuiKahuhipa akahai.

2 "Lawe hoi i kuu auamo, He oluolu io no, Mama maoli kuu ukana, No'u e maha ai oukou."

$\therefore$ Va pololi, ilihune, Hele mai i pomaikai : Nani ko ka uhane waiwai,

Ono kana ai maikai.

4 Na eha, maule, mai, mohole,

E na lawehala a pau, Hele koke io Iesu la, Lelepau, a ola mau.

5) Nau e hanai kou olnana, E. ke Kahuhipa mau, Ma ka poli o kou mana,

Nau hoomalu mau makou.

66.

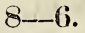

Ola ma o lesu wale no.

$1 \mathrm{KE}$ ola no ke mea maikai

No kanaka a pau;

Akahi waiwai makamae,

I waiwai mau kakou.

2 I noho nui hoi kakou,

Me ke Akua mau, 


\section{ILOOWELIVELS}

I ola mau ma kahi an,

Na uhane o kakou.

3 Pehea la kakou e ola'i

I luna o ke, ao?

Ia wai la hoi e hiki ai

I kahi ola mau?

4 Eia ke ola no kakon, lesu ka Haku mau, Ma kona aoa pololei, E hele ai kakou.

5 Aole Ola mana ae, E pono ai kakou ; Ke Kumu ia e hiki ai

I kahi da mat.

67.

$$
8 .
$$

Ololi ke ala e hiki akua i ke ola.

I I.ALLA ke ala $e$ iho ai Ilalo i ka make man; Auwe kolala huakai! He kini lehulehu no!

2 Ke ala pono e olu’i, He ololi, he pololei; ruku kona huakai, He nui nae ka pomaikai.

3 Hoole i kou kino no. Lapai $i$ kou bea no lesu, 
Haalele i na waiwai e, I loaa mai ke ola mau.

4 Ka me pauaho koke no, Haalele i ka pololei; Acle ia i hanau hou, $A$ ia ia no e make ai.

5 E kuu Alii, e hoomaikai, I kuu naau, i pono mau; E hooku paa, e alakai, A komo wau i kela 20 .

1 J.OHE' $A$ ka leo mai Sinai, Loaa kuu mea e hewa ai ; Haalulu kuu naau :

He leo weliweli no, "Iloolohe, a hoopono mau,

o make mau oukou."

2 Malaila wau i huli ai, Pae hou mai ka leo e, A weliweli au:

Ua ikaika mai ka olelo mau, "E lilo i ka hanau hou, O lilo, i ka po."

3 Poha ka leo kanawai, Kuu hewa hoi ua nui ae, Kaumaha maoli no: 


\section{HOOWELIWELI.}

A nana vau, u'akaka mai, "IS hanau hou e pono ai Na lawehala a pau."

4 Ua lohe au, ua haina mai Ko Iesu lanakila ae, Maluna o ka po: Aka, ua paa ia hua man, "E lilo i ka hanau hou" A pilihua wau.

5 A cha io, make au, Maalo ae ke Ola mau, Ua menemene no ; Hoohewa mai ke kanawai, $\mathrm{Na}$ ia la nae $\mathrm{i}$ wehe mai, A hanau hou mai au.

6 Pau kuu kaumaha, kuu makas, Ua ano hou na mea a pau,

Hauoli kuu naau, He make ma ke kanawai, He ola a be pomaikai, lloko o lesu.

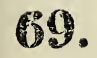

$$
7-6 .
$$

Ano ka la e ola'i.

1 U'OKI, ea! e huli mai, Mai hele hewa hou;

Mai paani ma ke kae

O ka pouli mau: 
Aia hoi ka make mau, No ka poe hookuli no Huli o hanle pu, Malalo i ka po.

2 Keia la e huli mai,

Hoolei $i$ ka naaupo;

Keia la, e pomaikai, li kii i naau hou:

E ka mex $i$ auwana nei, Huli mai, e hoomanao Ifele ma ka pololei,

A hiki i ke ao.

3 Iini mai na anela, E mibi kou naau, Kali mai no hoi Iesu, I kupu kou manao: Eia hoi! ua makaukau Kana ahaaina nei, Hele mai na mea a pau, Ia Iesu e ola'i.

Ile leo hoohuli $i$ ka poe bewa.

1 E MUI, e kanaka awwana nei, Noher la ka ikaika p bele $i$ ka po? Mahea la ke kino e pomaikai ai, Ke lilo ka whane, a make nau no? 


\section{HOOWELIWELI.}

2 E huli, e huli, o make oukou, Haalele oukou i na aoao kekee; Na lesu ke ola i hoomakaukau: Pehea la na uhane? e malie anei ?

3 Hea mai ka Makua, ke koi mai nei, E huli, e mihi, e hoopololei, Owau no ke Akua, he lokomaikai; No ke aha oukou la e make mau ai?

4 Kahea mai la Iesu, e hoi mai oukou, Na lubi, na eha, na pio, ra mai, Ua lawa ke ola no oukou a pau, No ke aha oukou la e make mau ai?

5 I mai la ka Uhane maikai ia oukou, Ua nele, ua hewa; e hele mai no, He nui kuu mana e maikai hou ai ; No ke aha oukou la e make mau ai?

6 Ano hoi e huli, o poino e, Kakali ko luna, e huli ano, E holo ia Iesu, o make menei; Paulele $i$ ke $A$ kua, e ola mau no.

6ra poino o ka poe i hoopanee.

1 AUWE ka poe i hoopanee, A mihi ole no!

Ua puni i ka wahahee, A make mau lakou. 
2 Ano e mili ai oukou,

Kauoha mai Iesu;

Manuli-wahi a lakou,

Alize, a huli no.

3 Alia, alia! mamuli no!

Ne e na alalai!

He mau pahele a ka po,

E make nui ai.

40 keia la kaawale no,

Ka la e ola ai;

I heia la, e lelepau,

la lesu ka Mohaj.

5 If mihi hoi, o make pu,

Mai kanalua hou;

Hoolilo io no Iesu,

O lilo i ka po.

6 Mamuli, pau ka leo nei,

I hea ia oukou;

Kokoke mai ka la hoopai;

E huli pu, ano.

72

8.

Ke koho ana $\mathrm{i}$ ke ola, a me ka make.

1 E KOHO no ka poe ata,

I ala po e make ai;

O kuu manao, e hooku paa,

Ma kuu Akua e ola'i. 


\section{HOOWHLIWELI.}

2 A wanao, e noi at, Ma kona noho-moi mau; A awakea, ini hou; A po ke ao, e pule no.

3 Nau, e ke Alua ola e, Kuu pule nei e lohe mai; Poino nae ka poe baakei, Ma kou huhu e make ai.

4 Nanea hewa no lakou, Ua lelepan ma keia ao, A ka, maluna kuu manao, Ma ko ka whane ola mau.

5 Maluna o ka Haku man, Kuu mau kaumaha nci a pau, Malaila no knu hilisat, Ma kona pono, e maha'i.

6 Ma liona lima, e paa ai, Ko lesu mau pokii a pau;

O nei kahua e ola'i, He nauwe ole, oia mau.

ํํㄹ.

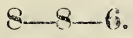

I keia po e kiiia maikou thene.

1 HAALELE, e ka hawawa,

I kau mau hana, kau aia;

E huli koke mai :

Kokoke mai ka make ou; 
I keia la, e hoomanao, Ano, o make e.

2 Pehea la kou uhane mauKou hewa lehulehu no, Ua kiekie e; Auhea kou waiwai oia mau, A komo i ka hale po, Kou kino make nei?

¿) Ina ka make ke kii mai, Aohe me e pale ae, He luna mana no; Kii mai e lawe ma ke ao, A $i$ ole oe e makaukau, E lawe i ka po.

4 Kou kino au e puni ai, E lilo i na ilo nae, I lepo maoli hou: Ina e mau ka hewa ou, E lanakila ma ka po, A luku oia mau.

5 I keia la, kahea hou, Kahea ka pono ia oukon, Na lawehala a pau;

I keia la, e mihi pu, Ano e holo io Iesu,

I ola oia mau. 
71. 8-7.

Ka Mesia ke Kahuhipa maikai.

1 HELE mai i ka Mesia,

E na lawehala a pau,

Na kaumaha, pilikia,

Hele nui ia Iesu,

L.clepau i keia Kumu,

Maha maoli no oukou.

2 Mama oluolu wale,

Ka auamo a Iesu,

Maha ko oukou uhane,

Pomaikai ke amo pu,

Mama, nani, hemolele,

Kona waiwai oia mau.

3 Nan'e hanai kona ohana,

Kahuhipa maikai mau,

A ma kona lima mana,

Hui na keiki hou;

Nan'e hali maluhia,

Ma ka poli ona no.

4 Nana no e kai malie,

Kona poe kaumaha no

A i hili, a hihia,

Nana e hoopono hou,

Ma ka pa o kona ola,

Malu oia mau lakou. 
5 Hapai pu mamuli ona,

Mihi, maha, malu man;

Lilo mai la kona ola,

No na hipa launa hou;

Kathu nani! Ahonui!

Nou, e lesu, nou makou.

75.

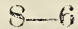

Aha aina eugnelio.

$1 \mathrm{E} \mathrm{KO}$ ke ao, hoolohe mai, Hanoli nui no;

Ua kani mai ka pu maikai,

$E$ hele mai onkou.

2 Ka poe e ai makani nei,

A maona ole no;

E na pololi me na mai,

E hele mai oukou.

3 Hoomalaukau mai nei Iesn, I ahaaina mau,

He ai, he waina, he waiu;

E hele mai oukou.

4 Ka poe maule makewai, Mai lilo i ka po;

Maanei ke kumuwai maikai;

E hele mai oukou.

5 Na Iesu no i wehe mai,

Ka puka e ola'i ; 


\section{HOOWELIWEL.}

Eia makou ke hele nei,

E komo pomaikai.

6 He ola e hauoli ai,

Na uhane i ke ao;

He ahaaina nani nei-

E hele pu kakou.

18.

8.

Ka polno o ka poe lookuli.

I POINO no na hoomalau,

Ke pale i ke ola mau;

He naaupo, he kuli no,

Ua koho maoli ma ka po.

2 He poe nanea hewa nei,

I lelepan ma keia ao;

Ua hoohei, a pepeni e,

I na uhane o lakou.

3 Pokole nae, pau koke no,

Ko lakou lealea nei, A uwe ka hope! I ka po!

Ka lua e uwe mau ai!

4 Na lahuikanaka a pau,

I hoopoina ia Iesu,

$\mathrm{Na}$ aina i hookuli mau,

$E$ auhulihia i ka po.

5 E Iesu, e aloha mai,

E hoopakele loa ia'u, 
Ia hope e uwe mau ai,

O ko ka aia poino mau.

$7 \%$

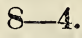

Ke kani ana o ka pu.

1 HAMAU! Ke kani nei ka pu,

Ka olelo maikai a Iesu ;

E holo ae mai o a o,

E lawe aku ana no,

Ka pono nui no kakou,

Ke ola mau.

2 Hoolohe, e ko lalo nei,

Na kanaka, na kamalii,

I keia leo nani e,

E malama, i loaa mai

Ka momi e pomaikai ai,

Ke ola mau.

3 E paio, e na koa e, E paio, i pakele ae,

A lanakila, kau ka lei; Me Iesu pu e noho ai, Ma kona aupuni iluna'e, Ke ola mau.

4 Malaila pu kakou a pau,

E mele a hoolea mau, Me na haipule a Iesu,

Me na anela $e$ hapai pa,

Ke mele nani a lakou,

Ke ola mau. 


\section{HOOWELIWELI.}

8.

Na hoomalau.

1 AUHEA oukou, na hoomalau,

Na kanaka o keia ao; Maluna no, e kau ae nei,

$\mathrm{Ka}$ inaina enaena e.

2 Ua hea mai, a hea hou,

$\mathrm{Ka}$ leo lani ia oukou,

E ala, wiki, holo ae,

'IKa puuhonua e ola'i.'

3 He leo lokomaikai nei,

No Iesu, ko oukou alii ;

Ina hooloheia mai,

E ola, a e pomaikai.

4 Ina kue, a hoopanee,

Pauaho kela, a pau e,

Ka leo hea ia oukou,

A iho oukou i ka po.

5 Pehea oukou o na aia?

Ka hope paha, keia la ; Hoopanee hou; e uhi mai,

Ka pouli e poino ai.

1 E MIHI, wahi a lesu, Mai kanalua hou; 
Ka mea hookuli, make no,

I kahi pouli mau.

2 Imua ona, e kulou,

E moe akahai,

E ae i ko Iesu manao,

Mai pale iki ae.

3 Kulou ano, o lohe e, Ke kani o ka pu, A pau ka wa e mihi ai, Ke hoopai mai Iesu.

4 He nani, a he ano e,

O kona aloha mai,

Ke hooloihi aku nei,

I keia wa maikai.

80.

8.

E kau ana ka make.

$1 \mathrm{KE}$ kau nei no maluna o'u, Ka malu o ka make mau,

A weliweli kuu naau,

He wiwo nui, a makau.

2 Hoao no wau e pale ae, He lima palupali nae;

Hou mai lka pua make ia'u,

Kupaka wau, a make mau.

3 Pau koke no, ko'u pomaikai,

E moe mau malalo ae, 


\section{HOOWELIWELI.}

O ko ke Akua inaina mau, Iwaenakonu o ka po.

4 Aole lohe hou ia mai, Ka leo lokomaikai ae, E hoonana i kuu naau, I wela i ke ahi mau.

5 Ka hope keia, ke hoomau, Ma ko ke kino, ko ke ao, Auwe! pehea? e ae anei, I keia make e kau nei?

6 E ala, e, e pilipu, E holo aku io Iesu, Ia ia la e hoolilo ai, I makamaka e ola'i.

81. $8-6$. Ua makaukau ke ola I AIA hoi! ke ku mai nei, Iesu ke 'Lii maikai, Ma kou naau ke kikeke, $E$ wehe $i$ komo ae.

2 Ma kona lima e paa nei,

Na mea e pono ai,

Oukou na ilihune, e,

$\mathrm{E}$ lawe wale ae.

3 I mai la oia ia oukou,

Ua pilikia wau, 
Ua make, a ua ala hou,

I pau ka make mau.

4 Iloko o'u ke ola mau,

Ka maha iluna'e,

A haawi wale aku au,

Aole e kuai.

5 Pehea? ke lawe nei oukou,

I keia pomaikai?

Ina hoole, ma ka po

Oukou e ilo ai,

82.

8.

Ohumu ana.

1 KA Haku e, palaka wau;

Hoohalahala kuu naau,

I ko'u noonoo, i ko'u nana,

Ka pomaikai o na aia.

2 He poe luna no lakon,

He poe hanohano no,

He nani ko lakou waiwai,

A lanakila, a haakei.

3 Aka, ka hope o lakou,

Ka hope weliweli no,

Auwe! auwe! ke ku mai nei,

Ma kahi nihinihi e.

4 Malalo ae, ke kaa mau no,

$\mathrm{Na}$ nalu ahi o ka po, 
Kokoke luu, a nalo ae,

Aole ike hou ia mai.

5 Aubea ko lakou nei waiwai ?

$\mathrm{Na}$ lealea hoi onei,

Ua lele ae, ua mae, ua pau,

A koe mai ka nele mau.

6 Pehea la, kuu uhane, e?

Hoohalahala hou anei?

Aole pono; e Iesu,

O oe no kuu waiwai mau.

1 POKOLE keia wa, Ka wa e hana nei;

E mihi e ka poe aia,

I keia wa maikai.

2 Pokole keia wa,

Kahea ia oukou,

E wiki, mai hookunana,

Paulele ia Iesu.

3 Pokole keia wa;

Hauoli oukou nei,

Ka Iesu Kristo poe kauwa,

Kokoke $e$ pomaikai.

4 Pokole keia wa;

Ke lele ae la no, 
Kokoke kakou e nana, $\mathrm{Ka}$ aina maikai mau.

5 Pokole keia wa,

F. kali iki ae, Alaila pii a noho paa, Me lesu iluna'e.

81.

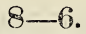

Ano ka manawa.

1 HOSANA i ka Haku mau,

A olioli pu,

Ke ola ko kaliou manao,

Ke ola ia Iesu.

2 E hele hoomaikai kakou, $\mathrm{Na}$ kona mau wawae,

Iehova, oia ke 'Lii mau,

O ko ke ao Moi.

3 Imua ona e kulou,

Mahalo akahai,

E lilo kakou nei a pau,

I mau kauwa maikai.

4 Ano ka wa; ke haliu mai,

O kona pepeiao,

E lohe, a ke kali nei,

I ko oukou manao.

5 E wiki, o hoa ia mai,

O kona huhu mau, 


\section{HOOWELIWELI}

A kau ka lima e hoopai,

$\mathrm{Na}$ uhane o oukou.

6 E wiki, o hoohiki mai,

Ke Akua o oukou,

Aole oukou komo ae,

I kona maha mau,

85.

$8-6$.

Naaupo ka mea hoopanee.

1 HOLOHE, e ka poe kue,

A hana hewa mau,

He leo lokomaikai nei,

Ke hea ia oukou.

2 Paipai ka leo ia oukou,

E wiki ano e,

E imi koke ia Iesu,

O kau mai ka hoopai.

3 He ino huhu e, ua kau,

Maluna o oukou,

Ke kanawai hoohewa mau,

E huli mai ano.

4 E wiki e, o nui ae,

Ka pilikia nei,

Ano ka la e malu ai ;

E wiki o pau e.

5 Ina alia a hoopanee,

Me e ka naaupo, 
Pau auanei ka wa maikai,

Pauaho hoi Iesu.

6 E wiki ea, mai lohi hou,

He pali imua ou,

Malalo ae ke kaa mau no,

Na nalu o ka po.

7 Malaila no e pau loa'i,

O koonei pomaikais,

Aole lohe hou ia mai,

Ka leo e ola'i.

86.

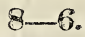

Leo hoohuli må.

1 E HULI mai, e huli mai,

Na lawehala nei,

Ke kau nei no ke kanawai,

Ke kanawai hoopai.

2 E huli mai, e huli mai,

Na ka Makua mau,

I hea mai, a kali ae,

I ko oukou manao.

3 E huli mai, e huli mai, Kauoha mai Iesu;

Mai noho a hoopaakiki,

I ko oukou naau.

4 F huli mai, e huli mai, Paipai ka Uhane maus 


\section{HOOWELWELI.}

Nai noho a hoole ae, la kumu o oukou.

5 E huli mai, e huli mai, Hea mai na hoahanau,

Hoolohe a e hui ae,

A hele pu oukou.

6 E huli mai, e huli mai,

Pai mai na anela,

E ku, nana, malalo ae,

He loko ahi a.

7 E huli mai, e huli mai,

E wiki ae oukou,

Kokoke pau ka wa paipai,

Kokoke mai ka po.

8 Ano, ano, e huli mai,

Mai panee hou oukou,

E panee hou? auwe! auwe!

Ko oukou make mau!

87.

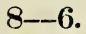

Ke ola a me ka make.

1 AIA maluna ma ke ao,

He lani nani e,

No na puali pono mau,

Puali pomaikai.

2 Aia malalo, ma ka po,

He lua make e, 
He lua pau, he kaula hao, No lanaka kue.

3 Malaila ka diabolo, Me kana poe kauwa, Uwe, kupaka, a, uwo, Aole loa e na.

4 Ke waiho nei ka lani mau, Imua o oukon, Gehena hoi, ke waiho pu, Mahea la ka manao?

5. Mahea oukou e koho ai?

Na makamaka, e, Ma ko ka lani pomaikai? Ma ko ka po anei ?

6 Hoomaopopo koke ae; $\mathrm{E}$ hele ana no, Ka wa maikai e koho ai:

E wiki ae oukou.

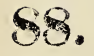

$$
\text { 8--7. }
$$

Popilikia ka mea hewa.

1 AUWE! hohonu e ka po!

Ke kau nei au maluna!

Loihi kahi ola mau!

Auhea hoi kuu kokua?

E apo mai, e Iesu, e!

O lilo kuu uhane nei,

I ko ka make lua. 
2 He lokomaikai kou manao,

I kii mai e hoohuli;

Popilikia loa wau,

I kuu naau hookuli :

Kokua mai, e lesu e,

O nalo kuu uhane nei,

I ko ka po pouli.

3 Kaumaha au i kuu aia,

Kokoke e palemo;

Me na pohaku kun kina,

Nou wale no ka hemo,

Hoolana mai, e Iesu e,

O make kuu uhane nei, Ano, aolc emo.

4 Eia'e ko kaua enemi,

Ua kii mai e kiola,

He nui no na mea ku e,

Auhea la kuu Hoola?

Hoola mai, e Tesu e,

O make mau kuu uhane nei,

Nou wale no kuu ola.

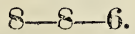

$\mathrm{K}$ a huli weliweli ana o $\mathrm{ka}$ mea hewa.

1 POINO nui ko ka po;

Pomaikai loa ko ke ao;

Ei'au mawaena no: 
A kali iki, i ole au

E laweia'e i kela ao,

E pio i ka po.

2 E paa mai, e kuu Haku, e, Ano, hoomalu koke mai,

O make au ano:

E alu mai, i koho au,

I waiwai no kuu uhane mau,

O nalo i ka po.

3 Hoike, i akaka mai,

Ke ano o kou la hoopai,

E kii mai ma na ao:

E hoopai i na mea ku e,

A ohi ae i kou waiwai,

Nau wau e hoomanao.

4 Hoomaikai e i kuu naar,

I ku paa ai ma kou aoao,

Ma kou mau kanawai;

E kiai makaukau no wau,

A pau kuu wa ma keia ao,

A hele pomaikai.

90. Noko lesu aupuni.

1 NANI ka lesu olelo,

Lohe mai e ko ke ao,

Eia hoi! kokoke mai la,

Ko ke Akua aupuni hou. 


\section{HOOWELIWELI.}

2 Huli mai oukou, e mibi,

E pau i ka hanau hou,

Hookaawale oiaio,

I ohua no'u oukou.

3 Malama i kuu kauoha,

$E$ aloha na naau,

Hooikaika mau e korno,

I he ola oia mau.

4 No na kanaka panlele,

Ke aupuni o ke ao;

O ka poe lohe ole,

No lakou ka make mau.

$5 \mathrm{E} \mathrm{k} a$ Uhane Hemolele,

Bapetizo ia makou,

Hoomaikai i na uhane,

No hou ckalesia mau.

9.

8.

Hoole ana i ke Akua.

1 OLFLO ko ka aia naa,

Aole no Akua mau:

Ua hewa pu no nac lakou;

Ua hokai pu lakou a pau.

2 Mai luna lilo o ke ao,

Ua nana mai ka Haku mau,

I ike i ka naanao,

O na keiki hoomalau. 
3 Ua pau lakou i ke kapae; Haumia pu ; ua kipi nae: Aole mea o lakou,

I hana ma ka pono no.

4 Ina e puka mai ia nei,

Ke Ola mai Ziona mai;

Ola na pio ia Iesu,

Iakoba ka $i$ hauoli pu.

92.

8.

Ka pomaikatoi me ka poino.

1 POMAIKAI ke kanaka ao,

I pale $i$ ko ka aia manao,

Haalele hoi i na haakei,

A hele ma ka pololei.

2 O kona olioli nae, Ma ko Iehova kanawai ; Malaila kona hoomanao, A ao ka po a po ke ao.

3 Anle kona lau e mae, He laau ma na kahawai, Ua hua mai ka hua mau, Pomaikai oia i ke ao.

4 He opala hoi ka poe aia, A ka makani $e$ puhi ai, Aole $e$ ku na mea kina, Me ka aha o ka poe maikại. 


\section{HOOWWLIWELI.}

5 Ike ka Lunakanawai, I ona pokii hoomaikai, Ka aoao o ka hoomalau, E make ia, e make mau.
Mihi.
$5-6$.
Ua hewa makou.
1 KO makou Alii, Ke Akua maikai;
E ike mai nei,
E maliu mai;
Ua lawa ke koko,
O kou mohai mau,
I pono ko loko,
A malu makou.
2 Ua kipi makou,
I ke Alii mau;
Paumaele mau no,
Ko makou naau:
Me e hoi ka mana,
O kona mohai;
O lesu ka Alana.
E ola mau ai.

¿ Ua hehi makou,

I kou kanawai,

A bolo mai no,

Iou la ke 'Lii.

No makou ka hewa; 
Nou hoi ka maikai;

No lesu ka eha, E malu mau ai.

4 Ko makou Moi, Mai ku e hoopai, I au mau pokii, E hoomaikai mai, Ma ko Tesu malu, E paa ai makou, Nau makou e alu, I pomaikai mau.

1.

8.

He mele mihi.

1 E KO makou Makua e, E hoomaikai ia makou nei;

I lilo ae makou a pau, I mau ohua hanau hou.

2 He naau ino ko makou, Ua kuli hoi na pepeiao, Ua hele hewa na wawae, Paumaele hoi na lima nei.

3 E Iesu, e hoolilo ae, Ia makou i kauwa maikai, I pau ae i ka hanau hou, Na naau kina o makou:

4 I lohe hoi na pepeiao, I mihi ko makou naau, 
I kuko hoi i kau maikai,

I waiwai nani e ola'i.

5 Alaila, hele pololei,

Hauoli i kou kanawai;

Hoonani $i$ kou Uhane mau;

Nana makou i hanau hou.

95. 8.

1 E KUU Akua maikai, e,

Imua ou e moe ai,

Kau kauwa hewa nui nei,

Mai noho a kipaku mai.

2 He pono kou, e kuu Alir,

He hemolele kou manao;

Aole olvolu mai,

I ke kina o kuu naau.

3 He hilahila maoli ko'u. He wiwo wale, he makau;

A komo mai ka hewa o'u,

Ke hoahewa mai nei ia'u.

4 Ua hana eha aku wau, Ia Iesu kuu Alii maikai; Ua hoomaunauna kuu naau, I kona aloha e ola'i.

5 Aka, ke kau nei kuu manao, Ma kona koko ano e, 
Oia kuu mea e uhi mai, Iesu kuu mohai e ola'i.

6 Ke Akua e aloha mai, No Iesu au i poni ai, Hookahi puuhonua nei, No'u, no ke kipi, e ola'i.

96. 8.

O lesu ka mea e pono ai.

I ALOHA oe kuu Maku, e, Kuu Paku, kuu Pohaku mau,

Kuu Ola e paulele ai,

Ka ikaika nei o kuu naau.

2 A loaa mai kuu hewa nei, Kaumaha loa kuu naau, Auwe! auwe! kuu make e! Ua kii mai no ka make mau.

3 Ua puni au i ko ka po, Haalulu au, a hoomanao, Kahea la wau i kuu Iesu, Ua lohe mai-ua ola au.

4 Aole loa poino hou, Ko Iesu lokomaikai mai, E hoomaikai mau kuu naau, I kona mana e ola'i.

$8 \%$

$$
\text { 8-8-6. }
$$

E noonoo ana ika mea palaka. 1 AUWE! ke noho nei no wau, Mawaena o ka make mau, 


\section{MIHI.}

A me ke ola nae:

O kuu naau ua paakuku,

Ua hoomaloka ia Iesu,

Ka mea e ola'i.

2 E Iesu, e hoano mai,

I kuu naau nanea nei,

I pono kuu manao:

Nau kuu uhane e hoomaemar

Ano, i keia la $e$ ola'i,

O make e no wau.

3 A hiki mai ka Haku mau,

Maluna iho o na ao,

I kela la hoopai;

E ku anei ilaila au,

Ma ko ka Moi lima akau,

E hoomaikaiia'i?

4 Kuu pono nui e hana'i,

E mihi-a e hilinai,

Maluna o Iesu,

A kii mai nei ka make ia'u,

Aole loa au makau,

E lanakila no.

98.<smiles>[3H][Hg]</smiles>

E hoopau ana i ka hewa

1 E KU e hou anei,

No kou aloha mai?

Pepehi hou anei makou,

I ka Moi Mohai? 
100

2 Papa mai, e ke Lii,

I ole ala hou,

Na hewa i kinaiia'e,

O luhi hou makou.

3 Na mea hooluhi mai,

Na hewa o kakou;

Ma kona kea ua kaulia'i,

Ua make ia lesu.

4 He ola ko kakou,

Aole pio hou;

Ua kuai oia, nona no,

I pokii ola mau.

99.

8.

$\mathbb{E}$ mihi io sna.

1 ILHOVA e aloha mai,

la makou nei, ua hewa no:

He pono mau kou kanawai;

He lohe ole ko makou.

2 Ua uhi mai ka naupo,

Ua hewa io ka naau,

E ahonui ia makou;

Kokoke i ka make mau.

3 Iehova, e hoola mai,

O make mau makou a pau,

Ho mai i naauao maikai ;

Ke mihi maoli nei makou. 


\section{MIII.}

4 Holoi i ko makou naau, 1 pau ka hala o makou; He koko mana ko lesu, E malu ai, i malu mau.

100.

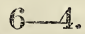

Ua lawehala na kanaka a pau.

1 UA ino paapu no, Na kanaka a pan; Ua hana hewa pu, Ua hina loa kakou:

Nolaila hoi e make ai, Ke kala ole ia mai.

2 IIokai anei kakou, I ke ola o Iesu?

A hana hewa mau?

A hele i ka po?

Namuli e poino no; Mamuli nele pu kakon.

3 Iesu, ke Ola mau, E lokomaikai mai,

Hoola ia makou, I mau ka pomaikai,

I lilo ole i ka po,

I loaa mai ko luna ao.

4 Aloha ia makou, Ka poe hewa nei, 
Haumia ka naau,

E huikala mai;

I ola makou nei a pau, A mele $i$ kou aloha mau.

101.

8.

Ka mohai o ka mea mihi.

1 ALOHA mai, kuu Haku e, I pau kuu hala nui nei, He lokomaikai nui kou, Ho mai i naau ano hou.

2 Imua ou, ke Akua, e, Kuu lawehala nui nei; Pakela loa kou maikai, Pela e huikala mai.

3 Mai lawe $i$ kou Uhanc mau; Hoouluhua hewa wau: Mai huhu hou i kau kauwa, Hoonalo nae i kuu kina.

4 Ka naau walania e, Kuu mohai e kaumaha nei; $\mathrm{Ka}$ naau mihi kau mohai, Aole ou haalele mai.

5 Ho hou mai i hauoli hou, No keia ola nui ou; A hai no wau i kou aoao, A huli mai na hoomalau. 
102.

8.

Ka mihi me ka haalulu.

1 KUU Haku, e aloha mai,

I ola kau kauwa aie;

Ma kou manawalea mau,

Hoopau i kuu aie a pau.

2 Ua hana hewa loa wau,

Aka, he ahonui kou,

He nui wale kou maikai,

lela, e kala nui mai.

3 Holoi i kuu uhane nei,

Nau kuu naau e hoomaemae,

Malaila ke kaumaha e,

He walania, he mihi nae.

4 Ke Akua lokomaikai mai,

Kuu mea i lawehala ai:

Mai kuu wa hou, ua hewa wau,

Ua hewa nui, hewa mau.

5 Ua kipi au i kuu Alii,

Ua haki ia'u kou kanawai;

Ina e pili, make au,

He pono kou, ke hoopai mau.

6 Hoola, e kuu Haku, e,

la kipi e haalulu nei;

Maluna ou kuu hilinai,

Ma kou aloha e ola'i. 
103.

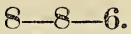

Ka manao o ka meai mihi io.

1 A $\mathrm{KAU}$ ko Iesu kanawai,

Ka pono ke akaka mai ;

O kuu mau hewa nae;

Hohonu ia me ka po,

Ua kiekie me ke ao,

Pehea e pono ai?

๘ He olelo oiaio nei, He pono ke paulele mai,

Na kanaka a pau ;

Kii mai la Iesu e ola'i,

Na lawehala nui nei,-

Ko lakou oi wau.

3 Ua ehacha loa wau,

I kuu mau hewa no a pau,

I make ai lesu:

O kuu manao aloha no,

Aole au e ku e hou,

Ina e make pu.

4 Ku hana, i kuu ola nei,

E hai i keia mea maikai,

I lohe ko ke ao,

Kii mai la Iesu e ola'i,

Na lawehala nui nei,

Ko lakou oi wau. 


\section{MIHI.}

104.

8-6.

He pule mihi.

1 KA Haku e, e nana mai,

I au mau kauwa nei,

Ua hana hewa, a kue

'Kou kanawai maikai.

2 Ua hoopoina ia Iesu,

Auwana na naau,

Ua hoomaloka, paakuku,

Palaka na manao.

3 Nolaila no e kau mai nei,

Kou lima e hahau,

A haule mai na ino e,

A kau ka make mau.

4 Imua ou ke kulou nei,

E Iesu ka Moi,

Mai noho a kipaku mai,

Ia makou na pokii.

5 E kuuia mai ka malu ou,

A uhi pono mai,

I nalo koke iho no,

Na hewa $e$ make ai.

105.

8.

No ka Hookeai.

1 AUWE! ua hewa maoli no,

Makou a pau imua ou,

I kela la, i keia la,

Mahuahua na kina. 
2 Ke hookaawale nei makou,

I keia la, i la noonoo,

I la nana, hooke ai,

Hoomake i ke kino nei.

3 Ke hele mai makou maanei,

Imua ou e kulou ai,

E ninau me ke akahai,

Pehea makou e pono ai ?

4 Haliu mai kou pepeiao,

E Iesu, ka Hoola mau;

Na hana $\alpha$ pau e pono ai,

Kau ia e hoike mai.

5 E hoohaahaa mai ia makou,

Hooeha mai i na naau,

I walania, a mıkau,

A holo ae iloko ou.

6 Ke mihi maoli nei ano,

$\mathrm{Na}$ hana hewa a makou;

Maliu mai, a launa pu,

Me kau mau kauwa, e Iesu.

106.

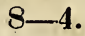

I naau hou e pono ai.

1. KE Akua e, e hana hou,

I ko'u naau, i pono mau,

Na hana, olelo, na manao,

Mai noho a haalele mai,

Ka Uhane ou, e pono ai,

O kuu naau. 


\section{MIIII.}

2 Ho mai kou lokomaikai e,

Ka mea e pomaikai ai;

E alakai i kuu wawae,

Imua ou, e Iesu e,

I lana e hauoli ae,

O kuu naau.

3 Pela e hiki pono ia'u,

Ke hai i na olelo au,

A paipai i ka poe kue,

E ala, a e huli mai,

E mihi, a e hilinai,

Maluna ou.

4 F Iesu e, e hoomaemae,

I kuu naau haumia nei,

Alaila, ike pono au,

Ka lokomaikai nani ou,

A mele, a mahalo pu,

Ke Akua mau.

$107.8-6$.

He hauoli, a me ke kaumaha.

1 HE nani no ko'u pomaikai,

A lana kuu manao,

I kela wa i nui ai,

Ke aloha ia Iesu.

2 Ua ike maopopo au,

Ke koko o Iesu,

Ua pili mai i kuu naau,

A pau ka hewa o'u. 
3 A puka mai ka wanaao, Hauoli kuu naau,

A po ka la ua mele au, Ke aloha o Iesu.

4 Ua pule au e launa pu,

Me Iesu, kuu Alii,

Ua olioli kuu naau,

I kona kanawai.

5 Aka, ano, ua ano e,

Ua emi ko'u manao,

A emi pu ko'u pomaikai;

Kaumaha ko'u naau.

6 A kau ka malu o ka po,

Uwe pouli wau,

A puka mai ka wanaao,

Aole laina no'u.

7 E Iesu e, e hoi mai,

Me kou Uhane pu,

I ala hou kau kauwa nei,

A noho lana mau.

08.8.

- Ka manaoio o ka lawehala e hele io lesu la

1 E HELE no wau ia Iesu,

Kuu Haku i alnha mai,

Ma kona wawae $e$ moe au,

Malaila, e pakele ai.

2 Eia no wau, e Iesu, e, 


\section{MIHI.}

E nana oluolu mai,

I make au i kuu aie,

He pono mau kou kanawai.

3 Ua ike au i kuu kina, Ke kuli, me ka hoomaau, Ka wahahee, a me $\mathrm{k} a$ aia; Nolaila, eha kuu naau.

4 He nui kuu me e hewa ai, I heluia, aole e pau;

Ua oi, e Iesu, kou maikai, Pakela kou aloha mau.

5 E kala mai i kuu aie, $\mathrm{Na}$ hewa nui o kuu naau; No kau olelo pololei, E ike mai-e ola au.

\section{6 -8.}

Ka hauoli lanı ma ka honua.

1 E NA haipule nei,

Hauoli pu kakou,

E mele pu, e hoomaikai,

A poai ia Iesu.

2 E mele ole ae

Ka poe naaupo;

O ko ke Lii mau pokii nae, Mahalo nui no.

3 Ke Akua o kakou, 
He weliweli nae,

Ko kakou nei Makua mau,

Ke Kahu e ola'i.

4 Ma ko Ziona puu,

$\mathrm{Na}$ hua ono no;

A hala, hele pu kakou,

Ma na ala gula mau.

5 E hele mele ae;

Aole waimaka hou:

Ko Emanuela aina nei;

Pakela kela ao.

110.

Pomaikai ke kanaka i makau ia lehova.

1 POMAIKAI wale o lakou, Ke mihi pono ka naau, Mahalo ma ke kanawai, A ma Iesu ka hilinai.

2 Ua huikala hoi lakou, Ua loaa mai ka naau hou, Ua alakai, ua noho pu, Ka Uhane maikai o Iesu.

3 Lilo ke $A$ kua o ke ao, No lakou i Makua mau, O ke 'Lii o na 'Lii a pau, Ke kaikuana o lakou.

$4 \mathrm{Na}$ malihini ilalo nei, 


\section{MIHI.}

Ua launa ole ia mai,

Aia ko lakou aina mau,

Iluna lilo o ke ao.

5 Na Iesu lakou i kiai,

A pau ko lakou hele nei,

E laweia'ku i ke ao,

Pomaikai i ke ola mau.

6 E Iesu, e, hoomaikai mai,

I na naau e pono ai,

I pomaikai makou a pau,

I loaa mai ke ola mau.

Me lesu ka maha.

1 KAUMAHA wau maanei,

He eha, luhi, mai :

$\mathrm{E}$ ake au e holo e,

Me Iesu e ola'i.

2 Me e ka waiwai nae,

Ko luna waiwai mau,

He maha, malu, pomaikai,

He ola loa no.

3 Ua mele nani mai,

Ko luna o ke ao;

Malaila mau kuu pomaikai,

Ke komo io wau.

4 E ahonui au, 


\section{A pau kuu luhi nei,}

E halawai pu me Iesu,

I kahi e ola'i.

10.6

Ka makemake e noho pu me Iesu.

1 EU kuu $u$ hane, e pii ae

Nana $i$ kou waiwai mau;

Waiho ka honua nei,

E lele i ke ao:

Aia hoi! ke emi nei,

Ko ke ao nei nani no;

Ala, eu, e holo e,

I kahi malu mau.

2 E hiolo koke no,

Na halelewa nei :

Aia hoi ka hale mau,

Me Iesu, e ola'i :

O ka uhane hanau hou,

$\mathrm{Na}$ ka Haku o ke ao,

$E$ ake ia e holo no,

I kona poli mau.

3 E na hoa luhi nei,

E lana ka manao;

Emo ole, hoi hou mai,

Ka Lanakila mau;

Ala, eu, a halawai

Me ke Ola no kakou;

Pau ka luhi; pomaikai;

E komo i ke ao. 


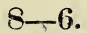

O lehova ka Moi.

1 E HAKANU, i ka Moi, Kulou na mea a pau: Haalulu wau ke mele nei,

I kona nani mau.

2 Ke ola, make, ke ao, ka po,

A $i$ ko ke Lii manao:

Ma kona noho moi no,

IEHova oia MaU.

3 Ma kana hana, ikea no,

Ko ia la mau manao,

O kana kuka nae, ua ko, Maanei, a me ke ao.

4 Pehea la wau ke pale ae,

I kuu Akua mau?

Ina poino,-pomaikai,-

Mamuli ou no wau.

5 A, ma kau buke ola-e!

O kuu inoa ke kau, Ma kahi haahaa loa nae, Malalo o Iesu.

\section{Hoahanau.}

\section{$114.8-7$.}

0 ka lesu malawaina.

1 KOMO, Iesu i kou mala,

Kuu mai hoi e ua mai,

O poho kou luhi ana, 
A maloo kou kihapai;

Make hewa hoi ka hana,

Ke ku e ka Mahiai-

E ka Haku, e hoolana;

Nou makou kokua mai.

2 Lawe ae i na pohaku,

O na naau ino nei;

Ko ke kino e kipaku,

Lulu ma ka pololei,

Pale oe i ka Satana,

E uhuki, a kinai;

E ka Haku, e hoolana;

Nau makou kokua mai.

3 Paipai $i$ na laau fiku, Me na kumu waina nei,

I mohala ke aloha,

Me ka pule hilinai;

Waiho hou me ke kipulı;

$E$ ahonui, a hua'e :

E ka Haku, e hooulu;

Nau makou kokua mai.

15. 0 ke kokua no ka poe popilikia.

1 E IEHOVA ke Akua,

O ke ao hoano mau,

Mai kou luakini luna,

E kokua ia makou;

E hoolohe i ka pule, 


\section{HOAHANAU.}

A ka poe ohaa ou;

Hoakea i au haipule,

Ku mai e ola'i lakou.

2 Ke kii nei ko makou hewa,

I ka make no makou,

A hihia, pilikia,

Eha nui na naau:

Nui wale kou aloha;

Mano ko lesu maikai,

Aia hoi ma kona koko,

Makou nei e malu ai.

: $E$ uli mai kela aloha,

I na hala o makou;

Aia no maluna ona

$N_{8}$ aie a keia a :

Nona ke aloha io;

Nou ko makeu nei naau;

Ia ia e ola'i na pio,

Pomaikai na kauwa au.

116.

$$
\text { 8-7- } 4 \text {. }
$$

Ke alakai o ka malihimi.

l E KAI oe, e Iehova,

I kou malihini nei;

Maule au, a mana oe,

$\mathrm{Na}$ kou lima e paa mai:

E ka Maue,

Hanai ia'u a maona mat.

2 Wehe mai i kumu ola, 
E hu ai ka wai maikai ; Hawi mai i kou aloha,

I paku, i alakai;

Kuu Hoola,

E hoolanakila mai.

3 Aia ku ma loredane,

Hoonana i kuu makau; Hoopae ae ikua uhane,

Ma ia kapa malu mau:

Haleluia,

Nou kuu mele oia mau.

$11 \%$.

8.

No ke ola ana o ka mai。

1 No Iesu ke aloha mau,

Ua lohe kona pepeiao;

Heaha ka'u e uku ai,

I kona lokomaikai mai ?

2 Nawaliwali iho nei-

Kuu kino hoi mai make e,

He mai, he hui, hə li makaw,

Ua weliweh maoli au.

3 Ua ehaeha kuu naan,

Mai lilo i ka make mau,

$\mathrm{Na}$ Iesu wau i lapaau mai;

Me e kuu mea e ola'i !

4 Pomaikai na ohua ou,

U'alohaia mai lakou; 


\section{HOAHANAU.}

Kuu ea au i kiai mai, Nou ia, e Iesu, e laa ai.

5 Nou loa kuu kino, kuu naau,

Kuu uhane hoi, me kuu manao;

Ka inoa ou, e hapai au,

I keia ao, i kela ao.

Ka lokomaikai o ka Haku.

1 E ALA kuu uhane nei, Hapai $i$ ka Haku e ola'i;

Eia kuu mea e oli ai,

Ka lokomaikai wale mai!

2 A make au i kuu kina,

Aloha wale mai kela;

Ua hoopakele wale mai,

Ka lokomaikai nui nei!

3 A nui mai na enemi,

$I$ alalai $i$ kuu hele nei;

Oia ke alakai nei ia'u,

Ka lokomaikai mana mau!

4 Pouli mai na ino e,

A nui, a hekili mai;

Ua ku mau mai Iesu me au;

He lokomaikai nani no!

5 Auwana hewa kuu naau,

Haalele wale ia Iesu;

Aole ona ano hou;

He lokomaikai oia mau! 
6 A hala mai ka pouli e, A maule hoi kuu kino nei, Kuu hanu make $e$ oli no, "Ka lokomaikai aole e pau."

7 E lele kuu uhane nei, E pii i kahi nani e, E mele, e mahalo mau, Ia lokomaikai, i ke ao.

Ka paio maikai.

$1 \mathrm{EU}$, na koa o Iesu; Me ka oi, e kukuku; Hele like; hahai pu, Ia Junakanawai : Oia ke Lii no oukou; Kei ka pono ona no, Kau ma ko oukou mau poo,

I lei e nani ai.

2 L-nakila kamahao! Auhee ia ia ko ka po; Hee ka lua-kupapau, I kona kea e :

No oukou ia Alakai; Ke Lii lanakila ai ; Kei k $\boldsymbol{a}$ Alihi akamai! Puukaua mana nei!

3 Eu, na koa waeia nei: Pale i na enemi; 


\section{HOAHANAU.}

Paio ma ka pololei,

Mamuli o Iesu ;

Ma ka lani, lelepau;

Hehi ae i keia ao;

Kaua no ke aupuni mau,

A lanakila pu.

4 Hoouka, e na koa laa;

Kinai ae i na kina;

Emi, pio ka aia,

O lalo o ke ao:

Hee Satana, a pau e-

Hoi oukou a kau na lei-

Mele, lanakila ae,

I ke Lii ola mau.

120 8-7-4.

No ka halawai ana o na hoahanat.

1 E NA hoahanau aloha,

Na Iesu i hoomaikai,

Nona ko kakou aloha,

Nona keia halawai,

E hoomana,

I ko kakou Alakai.

2 Nani kana kiai ana,

la kakou i malu ai ;

Nani wale kona mana,

'Hiki ai kakou ia nei ;

E mahalo,

I ke Alakai maikai. 
3 Imi pu i kana pono, Huli i kona kanawai ;

Lohe $i$ kana mau kauoha,

Pela e ku paa mau ai ;

E hauoli,

I ke Kaula pololei.

4 Ka ka Haku oihana,

E mahalo kakou nei,

Kona eha wale ana,

Ka kakou e mele ai ;

E aloha,

I ka Mohai e ola'i.

5 Pela ko kakou hoolana,

Launa io ia Iesu;

A pau keia noho ana,

Halawai hou i ke ao,

I.auna loa,

Aole hookaawale hou.

o ka maluhia o Ziona.

1 IEHOVA no ke $A$ kua mau,

Ka puuhonua no kakou;

Malaila mau e malu ai,

Ko Iesu Ekalesia nei.

2 Ina e olai nui mai,

Haalulu loa hoi ke kai;

Ziona, e, he malu kou,

Maanei Iehova me kakou. 


\section{HOAHANAU.}

3 'Ua kahe mau kou muliwai,

I ola kou kauhale nei;

Mawaena ou ke $A$ kua mau,

I ole ai e nauwe hou.

4 Kupikio na aina e,

$\mathrm{N} a$ aupuni hoi ua nauwe ae ;

Papa Iehova ia lakou,

Hehee ke ao a malu hou.

5 Iehova oia me kakou,

O ko Iakoba Akua no;

E hoomaikai na aina a pau,

I ko Ziona Ola mau.

122. 8-\%.

No ka huli ana i ke Akua me ke aloha.

1 HOI mai, e kuu uhane,

I kou wahi e maha'i;

Nani no ke ahonui,

O kou Haku e ola'i.

2 Nau i hoola kuu uhane,

Nou kuu wawae $e$ ku paa nei:

Pau ia oe kuu waimaka,

Pau kuu mea e make ai.

3 Hele wau i kona hale,

Me ko Iesu poe maikai,

$E$ oli $\boldsymbol{i}$ kona hemolele,

Nona no kuu pomaikai.

4 Eia wau mamuli ona,

Aole au haalele hou; 
Nona keia ola ana,

Nona no kuu ola mau.

5 E hoomana au ia ia la,

I kuu wa e ola nei;

A i kela noho ana,

Ia ia mau e hapai ai.

123.

$$
\text { 8-6. }
$$

Ka maluhia o Ziona.

1 HAUOLI, e Ziona, e, He malu pono kou; Pomaikai maoli kou Alii,

He Lanakila mau.

2 Kupikio na aina e,

Ooloku ke kai ;

Papa iho la kou Moi,

A malu koke mai.

3 Kauhale malu pomaikai,

No kou Akua mau,

He wai mau e hauoli ai,

$\mathrm{Na}$ hale lewa ou.

4 Ua ikeia ke $A$ kua mau,

Mawaena konu ou,

He lokomaikai aole $\boldsymbol{e}$ pau ;

Mai weliweli hou.

5 E ike pu na aina e, Iehova ke Lii mau :

Kulou, hoolohe, hoomaikai,

E ko ke ao a pau. 
8-6.

Ka makemake.e noho i ke ao.

$1 \mathrm{UA}$ olioli kuu naau,

E ake $e$ hele e,

$\mathrm{E}$ imi au i hale mau,

No kuu ubane nei.

2 Kaumaha wau e noho nei,

E kuko kuu naau,

I ka Iesu i kuai ai,

Ka lei, ke ola mau.

3 E pii e noho pomaikai,

Maluna o ke ao;

Malaila wau e maha ai,

Ma ke alo o Iesu.

4 Ua mele mai ka poe maikai,

E noho i ke ao;

Ua hanohano wale mai,

Ke kapa o lakou.

5 He mea iki wale no,

Ka eha make nei;

Pomaikai au ke loaa mai

Ko luna lei maikai.

6 E honikaika nui au,

Me kuu naau a pau,

A halawai ka uhane o'u,

Me Iesu i ke ao. 
$125.8-6$.

0 ka makemake e hele pu me ke Akua.

1 INA, me kuu Akua mau,

Kuu hele launa pu!

A maamaama kuu aoao,

E hiki ia Iesu.

2 Auhea la kela pomaikaj,

I kau ma kuu naau,

No Iesu, i kuu hilinai,

Ma kona pono mau?

3 Kei kuu mau hora malu e!

Maikai ka hoomanao!

He nele, eha, koe mai

He ole keia ao.

$4 \mathrm{E}$ hoi mai, e ka Uhane Laa,

Ka Luna e maha'i;

Ua mihi au i kuu kina,

Kou me i hele ai.

5 Ina he kii no kuu naau,

He akua wahahee;

Kokua mai e pale au,

A nou kuu hoomaikai.

6 Alaila, me ke Akua mau,

Kuu hele launa pu ;

A maamaama kuu aoao,

E hiki ia Iesu. 


\section{HOAHANAU.}

\section{$6-8$.}

Na oihana nani e pono ai ka ekalesia.

1 KOU nani, e ke Lii,

Hoike keia ao ;

$\mathrm{O}$ au haipule ponoi, Mahalo pu lakou.

2 Ua ku hauoli no,

Ma ko Ziona puu,

$\mathrm{Na}$ hanai au, hapai lakon,

Hauoli nou, Iesu.

3 E poai makaikai,

Na malihini hou,

I ko ke Lii kauhale nei,

E noho ai makou.

$4 \mathrm{Na}$ oihana nae,

I luakini ou,

Na mele, pule hoomaikai,

A hai maikai lakou.

5 He pono, naauao!

He nani, pololei!

$\mathrm{Na}$ nani nae o keia ao,

E nalo koke nei.

6 Ko kakou Alakai,

Ke $A$ kua mana mau :

No kakou ia malalo nei ;

No kakou i ke ao. 
Ka halawai hoahanau.

1 ALOHA, e na hoahanau, Iloko o Iesu ; Hauoli ko kaknu naau, A launa pono pu.

2 No Iesu keia halawai, No Iesu na naau; Mahalo i ke Alakai; He ahonui mau.

3 Auhea ke Lii maikai e ae, Auhea ka like pu? Maanei kakou e ku paa ai, Mamuli o Iesu.

4 Pehea la kakou e ola'i, Ke hoomaunauna hou, I kona ola nui nei? He aloha oia mau.

5 Aloha ino o Iesu; Ka Makamaka mau; Ua hana, luhi, make no, I ola mau kakou.

6 Malaila ko kakou manao, I keia ao a pau, A halawai hou i ke ao, Malaila mele mau. 


\section{HOAHANAU.}

128.

8.

Ka mele kanikau.

1 MA ko Babela muliwai,

Kakou i noho iho ai,

A ia Ziona $i$ hoomanao,

Ua uwe kakou, ua kanikau.

2 Ua kaulia'e i na wilou,

Na mea kani a kakou;

Kaumaha ko kakou naau;

Ierusalema ka manao.

3 Ka poe i lawe pio ai,

Ia kakou nei, ua olelo mai,

"E oli mai nei ia makou,

I ka Ziona mele no."

4 Pehea makou e mele ai,

I ka Iehova mele e,

I kahi malihini nei,

I keia aina akua kii ?

5 Ina poina iki ia'u,

Ierusalema i pau ai,

E lolo e kuu lima akau,

A pili kuu alelo nei.

6 Iini au e ike hou,

Ia luakini o Iesu,

A malu hou na hoahanau,

Malaila kuu hauoli mau. 
$1206-8$.

O ka hoolana ana i na haipule pilikia.

1 E LAWE nui mai, Mai keia mau wilou,

I ka oukou mea kani nei,

Hookani ia Iesu.

2 Ma keia aina e;

I malihini ai,

Kokoke mai ka hale mau,

$\mathrm{O}$ ke Lii pomaikai.

3 Aole e hoolei,

I kona mau pokii ;

Ina ike $a$ ka pouli e-

Paulele i ke Lii.

4 He hale oi $a$ mau,

E malu nui ai,

Na malihini lelepau

I keia Alakai.

5 Ina kaumaha pu

Ko kakou mau naau; He lokomaikai ko Iesu,

Aole no e pau.

6 A pau ka luhi nei, E hele mele pu,

Ma kona Luakini e,

Hoonani mau kakou. 
$6-4$.

O Iesu ma Kal'vari.

$1 \mathrm{KE}$ nana aku nei Ke Keikihipa e, Ma Kal'vari,

E lokomaikai ia'u, Kuu hewa e hoopau, Hoomaemae $i$ kuu naau, Iesu ke'lii.

$2 \mathrm{E}$ hawi wale mai Ka ikaika, ka maikai,

No kuu naau ;

Ua make oe no'u, Ho mai iloko o'u,

I naau ano hou, Ke aloha mau.

3 A hele aku wau, $\mathrm{Ka}$ waoakua mau

O keia ao,

O oe kuu Alakai, Kokua a kiai,

I ole e auwana'e, A make mau.

4 A pau kuu ola nei,

A kaa ka make e,

Maluna o'u,

Alaila, e Iesu,

Hoopau i kuu makau, 


\section{Kaikai a hompae i’au}

Ke ola mau.

131.

7.

O lesu ka mohai e ola'i.

1 E Iesu ka mohai no'u,

Holo au iloho ou,

Kahe mai kou koko mau,

Me lia wai mai kou aoao;

Oia no ka punawai,

Kahi a'u e macmae ai.

2 Ina uwe waimaka wau,

Ina hooikaika mau,

Aole oia he mohai

No ka hewa e pau ai,

Oe ke hoola nei,

Aole mohai hala e.

3 I kuu wa e ola nei,

I kuu wa e make ai,

A pii ae i kela ao,

A nana $i$ kou nani mau;

E Iesu, ka mohai no'u,

Holo au iloko ou.

328 .

Kakau ia ka inoa ma ka buke e ola'i.

1 A mapopo lea ia'u, O kuu inoa ua kau, Maluna ma ka buke au, Ka buke ola mau: 
Alaila pio kuu makau,

A olioli ae,

Pau kuu kaumaha, a maloo

O kuu waimaka nei.

2 Ina kue mai ko ke ao,

I kuu uhane nei,

A pana mai o ko ka po,

Na pua e make ai,

Alaila wiwo ole au,

E pale ikaika mau,

I ko Satana ma huhu,

A me ko keia ao.

3 Ina e haule paapu mai

$\mathrm{Na}$ pilikia e,

$\mathrm{Na}$ ino e kaumaha ai,

A pouli wale ae,

Na Iesu e kokua mai,

I lanakila wau,

A hoopae oluolu ae

I kahi maha mau.

4 Malaila no e auau ai,

Kuu uhane luhi nei,

Ma ko ka lani wai maemae,

A maha nani $\mathrm{c}$,

Aole hoi e kaa hou mai,

Na nalu o be ao

Maluna o kuu uhane nei,

I kahi malu mau. 
Mele oluolu i ka Haku.

1 E na malihini nei, Na keiki a ke 'Lii, Naue me ke mele ae, Ma ka waoakua nei.

2 Mele aku ia Iesu, Mele a mahalo pu, Nani kona mau aoao, Nani kana hana $\boldsymbol{a}$ pau.

3 Naue me ka heomanao, 'Ko na kaula mau aoao, Hele pololei lakou, Make, a pomaikai mau.

4 Hoohalike me lakou, Ahonui a kuoo, Aneane hiki mai O ka wa e hoomaha"i.

5 E ka ohana pomaikai, Mai makau; e hilinai, Ma ka poli o Iesu, O ke kahu maikai mau.

6 E ka Haku, e ao mai, I au mau malihini nei, E hoopau i na aoao, A hahai mamuli ou. 


\section{HOAHANAU.}

$6-8$.

E makaala mau.

1 KUU uhane e kiai,

He lehulehu no

Na piliki $a$ e tihia'i,

Na hewa o ke ao.

2 Ke keakea nei

Ia poe enemi,

I ole $\boldsymbol{e} e$ e hiki ae

'Ke ola maluna' e.

3 Nolaila, e kuoo,

E paio, pule mau,

Hoouka, a hoouka hou,

I hee ae ko ka po.

4 Ina i emi mai,

Ka hana, ka manao,

E wiki i ke noi ae,

E kokua mai Iesu.

5 Mai wehe koke ae

'Ka aahu kaua ou,

Mai kuhi hoi ua loaa mai,

Ka lanakila mau.

6 Aole loa e pau,

Ke kaua $i$ kaika ou,

A pau ke ola nei, a hau,

Ka lei maluna ou.

7 E kuu uhane, e, 
E hooikaika hou, A make ae ke kino nei, Alaila maha mau.

135.

$$
\text { 8-6. }
$$

Ka malu e pornaikai ai.

1 A PAU na la, a uhi mai,

Ke kapa make o'u,

Alaila wau e hoopau ai,

I ko onei manao.

2 Ma kahi mehameha no,

E hele aku ai,

E pule, a e launa pu, Me Iesu, kuu Moi.

3 E ake au e hoomanao,

I kona aloha mai,

A noi i kokua hou, Ma keia hope ae.

4 Maluma ona e kau ai,

Na pilikia o'u,

Kuu eha, kuu kaumaha nei,

Na ia la e hoopau.

5 Ke lana nei no kuu manao,

Ke nana iluna'e,

I kahi maamaama mau,

E maha mau loa'i.

6 A po kuu la e noho nei,

E ake kuu naau, 


\section{WOAHANAU.}

E kaa mai keia malue, I kela malu mau.

Hoonani i ka Haku mau.

1 HOONANI, na pokii,

I ko oukou Alin,

Ka Haku mau;

$\mathrm{Na}$ malihini nei,

E pii houoli ae,

I hahi e maha",

Ziona hou.

2 Na lesu ka mohai,

Oukou e alakai,

Ma keia ao;

A hiki ae onkou,

I kahi maha man,

Ierusalema hou,

Nemele man.

3 He nani e na lei,

I ulanaia mai,

No onkou nei;

Ka lei, ka pomaikai,

Ka pau loa ana mai,

O na kaumaha nei,

Pomaikai e.

4 E naue ae oukou,

Na kona mana no,

E alu pu; 
Nana hauoli ae,

I kahi e ola'i,

Kikoo a hoopii ae,

'Ka lani mau.

$12 \%$

7.

o Iesu ka puuhonua.

1 E KA Puuhonua mau,

O na uhane o makou,

I ka wa e poi mai nei,

O na nalu, ino e,

Ia makou, a mai poho,

Nau e uhi, nau e uhi,

Nau e uhi ia makou.

2 Ala mai na mea kue, Paio mai na enemi, Huna, Iesu, huna mai, Ia makou iloko ou, A kaa ae na ino nei, I pae ola, i pae ola,

I pae ola i ke ao.

3 Aole puuhonua e, No makou e malu ai ; Pilikia no makou, Maule, a mai make no, Holo nae iloko ou, Malu loa, malu loa, Malu loa, malu mau. 


\section{4-6-8.}

Thooikaika wivo ole

1

ALA'E kakous,

Na hoahanau.

Hoolei i na makau;

Ala'e a holo pu kakou,

$I$ ho ha lani puka mes:

Malaila no,

Ka waiwai mau,

L. lana ka manao.

Ke prapu nei,

Na nea kue,

He ala ololi;

E hooikaika nae kakou,

Inahi mamuli o lesu,

Ke alakai.

Es pono ai,

To i a la hakai.

Eluhi no,

A maule pu,

Ka poe kuihe,

A lilinai maluna no

O ka ikaika o lakou;

A lelepau

Ke Akua mau,

Alaila, ala mai.

4

E lakou nei,

E ala e, 
Enana iluna'e,

Ma kahi no e waiho ai,

Ka waiwai mau, na lei maikai,

No kakou no,

Ke hana mau,

Na mea e loaa'i.

5 Ina kakou,

E naue pu,

I kahi e loaa'i,

Na holoku alohi mau,

Na lei olinolino pu,

A loaa mai,

E waiho nae,

Ma ko Iesu wawae.

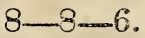

Ka Haku ma kona libapaí

I KA Haku e, e iho mai,

Maloko o kou kihapai,

Hooua nui mai,

I ulu mai a mohala'e,

Na hua i luluia mai,

A pua a maikai.

2 Nahele no kou kihapai,

Maloo a ulu wale ae,

Na ino paapu e;

A tho mai ka Uhane ou,

Iuai iwaenakonu no,

He punawai maikai. 


\section{IIOAHANAU.}

3 Malaila no e hoomau ai.

A kupu ae na hua maikai,

Houoli na naau;

Malaila hoi e inu ai,

Ka poe luhi, make wai,

A pohala'e lakou.

4 O makou nei kou kiha 'ai,

E launa pu, e paipai uan,

I na lala maloo;

Ekomo kou Uhane mau,

Iloko o ko lakou naau,

I ulu hou lakou.

5 Maluna nae ke kihapai, I paapu i na pua maikai,

Ka Paredaiso mau;

Ini ko makou naau,

E komo ae a noho pu,

Ne ke Lii o makou.

6 Malaila no e hui pu,

Me na puali o lesu,

He lehulehu no,

A lawe a hookani mau,

Na lira gula kamahan,

A miliani pu.

10.

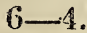

Manao Lina.

1 POULI keia ao,

Kaumaha maoli no, 


\section{Malalo nei;}

He mani kela ao,

Pomaikai oia maur,

Malaila kuu manao,

Me kuu Moi.

- Iesu kolaila la, Kolaila momi laa,

He ao mau no;

Ua lana kuu manas.

E pii iluna no,

E noho loa pu,

Me kuu lesa.

3 Eia ke noho nei,

Ma kahi paakiki,

Malalo nei ;

E ake knu manao,

E ike maoli no,

A noho loa pu,

Maluna ae.

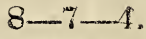

Haku loa.

1 HOKU loa, ano e,

Lilelile a maikai.

Kau iluna ma ke ao,

Lama no na aina a pav,

Hoku loa, hoku mau,

F. kokua ia makou. 
2 Kamahao ka mailiai ou,

E ka Haku o ke ao,

Mana, ike, akamai,

Ola lanakila hoi;

Lanakila, oia mau,

E hoola ia makou.

3 Emi i ka nani ou,

Fa hoku mai o a o,

Pio e na lama a pau,

Ke hoea mai lesu,

Hoku loa, ano e,

E manao mai ia makou.

\section{Sabati.}

1

\section{8.}

O ka mahalo ana i ka la boano o lehova.

1 HAUOLI i ka la maikai;

E hapaina ke Akua mau;

I keia la, ku hou mai ai,

Ka Lanakila kamahao.

2. Hoano maoli pu kakou,

Ia la hoano no Iesu,

He kapu e hauoli ai,

No ko ka uhane ponaikai.

3 Hiilani nui keia ao,

I ko Iehova pono mau,

I hoano lokomaikai mai,

I la hoano e maha'i. 
4 Hoomaha i ka luhi e,

E huli waiwai e ola i,

I keia la, e lelepau,

Ma ko Iesu cupuni mau.

5 Hoomana pu, e ko ke ao, A pau na kapu nei a pau,

He maha mau ke waiho mai,

No kona kini pomaikai.

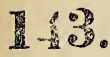

$$
4-6-8 \text {. }
$$

O ka mahalo i ke kakahiaka hoano.

1 ALA'E oukou!

E mele pu,

1 ke Akua mau;

Ke alaula hou ua wehe mai;

He nani no ke kapu nei!

Ala'e kakou,

Aloha hou,

I ke Lii o ke ao.

2 Ia la maikai,

I ku mai ai,

Ke Ola nani mau,

He Lanakila mana no,

Mauma ae o ko ka po,

Ua puka no,

Ka hoku ao,

A ala hou Iesu.

3 I keia wa,

Na anela, 


\section{SABATI.}

I lele nani mai,

Fike i ka Haku mau,

Ua ola hou, ua pahaohao:

A hoi lakou,

I kela ao,

E hai hauoli ae.

14. 8 . 8 ka hauoli ana i na hana hoano.

$1 \mathrm{HE}$ hana e hauoli ai,

Ke mele me ka hoomaikai,

E hai i kou aloha mau,

E ke'lii pono o ke ao.

2 He nani kou la kapu nei,

E waiho ai na hana e;

E hapai $i$ kou inoa mau,

Kuu leo pu me kuu naau.

3 E olioli nui au,

I kau mau hana nani no;

Hohonu maoli kou manao!

Kupaianaha kou aoao!

4 E pau no kou mau enemi,

P'uehu nui na haakei,

Ua ulu me na lau lakou,

A mae, a make, i ka po.

5 A hemolele kuu naau,

Hauoli au ma kela ao,

Malaila mau e mele ai,

Malaila mau kuu pomaikai. 
145.

No ke ola i haiiit $i$ ko Ziona poe pio.

1 HE nani kona mau wawae,

Ko ka Mesia luna nei.

I hai mai i ka malu hou,

He ola keia no kakou.

2 "Ziona! e hauoli hou!

"O kou Alii ke Akua ou;

"He Lunakanawai maikai,

"Ka Lanakila e ola'i.

3 "E ola! e na pio, e!

"Ua pau ka luhi hewa nei;

"Ma ke Lii mau, e malu ai,

"O hoi pu, a pomaikai."

146.

8.

Hauoli ika la Sabati.

1 UA hiki mai ka la maikai, He pomaikai no kakou nei;

Hoolea i ke'Lii o ke $\alpha$,

No kona lokomaikai mau.

2 No ke Akua keia la,

Hookapu loa mai kela,

I la hoano nona no,

I la hoomaha no kakous.

3 E olioli pu kakou,

Hauoli maoli ko ke ao,

I ke'Lii i haawi mai,

I Sabati e malu ai. 
4 E himeni aloha pu,

E pule i ka Haku mau,

Hoolono $i$ kona kanawai,

Hapai $i$ ka leo e ola'i.

5 A pau na la hoano nei;

He maha koe i ke ao,

He la hoano ano e,

He kapu maoli oia mau.

$1 \%$

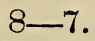

No ḳa hele ana $\mathrm{i}$ kauhale o Ziona .

1 NANI loa kou ka hale,

E lehova kuu Mui,

Iini wale kuu uhane,

Me ke'Lii e noho ai.

2 Pomaikai kou mau haipule,

Ma kou hale ma!u nei,

Nui ko lakou mahalo,

I kou lokomaikai mai.

3 Hele mau lakou imua,

Ma kou ala pololei;

A imua o lie Akua,

I ka lani e ku ai.

4 Oe ka La, a me ka Malu,

No ka Iesu huakai ;

Ma ka po, a ma ka wela,

Nou lakou e pono ai.

5 E Iehova ke'Lii nani, 


\section{Lohe i kuu pule nei ;}

Nau hoomalu kuu uhane,

Ma kou lani e ola'i.

148.

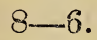

E hoommao i ka la Sabati a e hoano ia.

1 ILHOVA ke Akua mau,

Ka Lunakanawai,

A nana i hoano no,

I keia la maikai.

2 E hoomanao i keia la,

I la hoano no;

I oluolu mai kela,

I pomaikai kakou.

3 Ko Iesu la i ku mai ai,

I lanakila hou;

Ka kakou la e huli nei,

I ola no kakou.

4 No Iesu ko kakou manao,

I kona kapu nei;

Iraalele wale hoi kakou,

I na manao ku e.

5 I nui mai ka ikaika mau,

I like mau kakou,

Me na haipule o Iesu,

Maluna o ke ao.

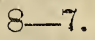

He oiaio, maanei no Iehova.

$1 \mathrm{HE}$ wahi eehia nei,

Maanei ke Akua nani; 


\section{SABATI.}

O kona hale laa maikai, Ka puka o ka lani :

Hauoli mai na anela, Ke komo pu na kanaka, I aha mililani.

2 He Betela e noho ai, Ka Uhane Hemolele;

Maanei ka mana e ola'i, $\mathrm{Na}$ lepero paulele:

Na mai, na kuli, na lolo, Maanei ke ola no oukou,

Kii koke mai, o nele.

$3 \mathrm{E}$ noho launa mai anei

Iehova Sabaota,

Me kanaka enuhe nei?

Me e no ke aloha!

F akahai, e ko ke ao,

Hauoli weliweli pu,

Hoolilo nui nona.

O ka mahalo i ka la Sabati o ke Akua.

1 NANI keia Sabati!

Kani kona bele nei,

Me na kumu a kakou,

Halawai, hoomana hou.

2 Hele pu na kamalii,

I ke kula Sabati,

Imi i ke ola mau,

Mele like ia Iesu. 
3 Eia hoi, kokoke po, Keia la hoana no;

Koe no ke aupuni mau,

No ko Iesu poe a pau.

4 Aia hoi, he nani e, Ko ka lani Sabati;

Nani no na mele mau,

Ma ke alo o Iesu.

5 Ina hiki pu kakou,

I ko luna kapu mau,

Me na anela maikai,

Mele lanakila ai.

6 Aia mau ka pomaikai,

Me ka Haku e ola'i,

Ola mau, a mele pu,

$\mathrm{Na}$ haipule o Iesu.

1รั1. 8.

O ka makemake e noho launa pu ure I esu.

$1 \mathrm{HE}$ pono no kakou maanei,

Me Iesu pu e noho ai,

A lilo ko kakou naau,

I luakini nona mau.

2 E komo mai, e Iesu, e!

Iloko o kou hale nei ;

E noho mai a launa pu,

I pomaikai mau ai makou.

3 Hoike i kou nani mau,

Ma keia luakini ou, 


\section{SABATI.}

I pumehana na naau,

Me ke aloha nui nou.

4 Hoopahaohao mai ia makou,

I like me ke ano ou,

I makaukau e noho mau,

Me au haipule i ke ao.

5 Maanei e launa ai kakou,

A pau na hana nei a pau,

A kuu ka make ia makou,

E launa mau i kela ao.

152.

$$
6-8 .
$$

Launa hauoli ma ka luakini.

1 HAUOLI wale au;

Olelo mai lakou,

I keia la e imi aí,

I ke Akua mau,

Ma ko Ziona puu,

Malaila no e hoomana'i.

2 Ke naue oli nei,

I kahi $e$ lohe ai,

$\mathrm{Ka}$ olelo maikai a Iesu;

Pomaikai wale ou,

Hauoli kuu naau,

Me Iesu au e launa pu.

3 Maanei e pule ai,

A me ka hoomaikai,

Na kauwa pono a Iesu;

Kaumaha wale nae, 
Na kanaka kue,

Haalulu, a makau lakou.

4 He maluhia mau,

Ma ko Iesu Heiau,

Maanei no au e noho ai ;

A launa me Iesu,

A me na hoahanau,

I nui ai huu pomaikai.

5 A pau kun noho mai,

Ma kona hale rei,

Alaila pii a komo ae;

I kela hale mau,

Maluna ma ke ao,

Malaila mau e hoomana'i.

雷的。

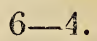

Ka makahiki fubile.

1 PUHI $i$ ka pu oukou,

Hookani maikai ae,

I lohe ko ke ao,

I keia pomaikai,

Ua hiki mai ka Iubile,

E ola, e na pio e!

2 Hapai i ka Mohai, Ke Kalahala mau,

He koko makamae,

E malu ai ke ao,

Ua hiki mai ka Iubile!

E ola, e na pio e! 
3 E na poino, e,

Na kauwa a ka po,

Na paa i na aie,

E ola ia Iesu;

Ua hiki mai ka Iubile,

Ou hoi oukou na pio nei.

4 Hoolohe mai oukou,

Ia leo e ola'i!

E holo ia Iesu,

Ke'Lii e malu ai!

He makahiki Iubile!

E ola, e na pio, e!

5 E hoi pu kakou,

I kahi e ola'i!

I kona aina mau,

E noho pomailiai ;

Maluna mau he Iubile,

E ola mau na pio, e!

\section{Pule hooman.}

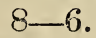

Ka iho ana mai o ka Uhane.

1 KA Uhane mau, ua hiki mai,

Ke huli mai nei no,

Na kanaka, na kamalii,

Hauoli pu kakou.

2 Ka poe hoomaloka no,

Ka poe hoomalau, 
Ke weliweli nei lakou,

Makau o make mau.

3 Mai luna mai, ke kanawai,

Haalulu ae lakou,

- Malalo no, o kaa mau ai,

Na nalu o ka po.

4 Nana lakou, a holo ae,

Iloko o Iesu.

I ole e kau koke mai,

Ko ke Aku $\alpha$ huhu.

5 Eia lakou ke noho nei,

Ma keia halawai,

Ke mihi nei, ke hoopau ae,

I ko lakou kue.

$6 \mathrm{Na}$ anela, na kanaka,

Hauoli pu oukou;

Ua hiki mai i keia wa,

IKa Uhanc mana mau.

15. 6 -8.

Hauoli no ka huli mai o kanska.

i He olioli e;

Ua huli io mai,

Kekahi lawehala nei,

Mai kona hewa mai.

2 Hauoli pu kakou,

Pomaikai oia nei,

Auwana no a mai poho,

A loaa hou mai nei. 


\section{PULE HOOMAU.}

\$e lawe nei Iesu,

I keia hipa hou,

lloko o kona lima mau,

- E lilo nona no.

4 Ko luna poe maikai,

Hauoli pu lakou,

Ua nalowale oia nei,

A hoi hou mai no.

156. 8.

Ke huli mai ua kanaka.

1 NANA kakou, mai o a o,

Ua makaukau na kihapai,

Ua hiki mai ka Uhane mau,

Ke ulu nei na hua maikai.

2 He hana kupanaha no,

Ka hana a ka Uhane mau,

Ua hiki mai ka Iubile,

Ke hoi mai nei na pio nei.

3 Ke huli like nei lakou,

Ke mihi nei, ke kanikau,

No ko lakou mau hewa no,

A hoi aku ia Iesu.

4 Auhea oukou e na kue,

E huli koke mai oukou,

O po ka la, á iho ae,

I kela po pouli mau.

5 Ano ka wa e huli ai, Ka wa e noho pu mai nei 
Ka Uhane, e kokua mai;

E wiki o poino e.

157.

\section{8.}

Ke huli mai na sia.

1 HAUOLI pu, Iesu ke'Lii, Ke iho lanakila nei ;

Ke huli mai la na aia, Ke hemo ae na mea paa.

2 Ke ae lakou, o Iesu no,

Ke Ola nui no lakou;

Ke hele nei ko o, ko onei,

I ko Ziona puka nei.

$3 \mathrm{Ka}$ poe auwana wale no,

Ka poe hihia a poho,

I na pahele a ka po,

Ke huli mai nei no lakou.

4 E Iesu, e hoonui ae,

'Kou lanakila ana mai ;'

Hoopio i na kipi nei,

E hoohaahaa i na kue.

5 Ko lalo, me ko luna pu,

$\mathrm{E}$ haleluia ia Iesu,

No keia huli nui mai,

O na auwana, kipi nei.

158.

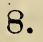

Hzaluln ka poe bewa.

1 HOOLOHE e, ka poe mai,

Ka leo make maluna'e, 


\section{PULE HOOMAU.}

Haalulu wale na kue,

Makau, kaumaha, a uwe.

2 Anapu mai ka uila e, Mai loko o ke kanawai,

Hamama no ka lua po,

Kokoke lakou e poho.

3 A poha mai ka leo e; Mai luna mai o Kal'vari,

He leo lawe, no lakou, 'Ka malu me ke ola mau.

4 Hauoli ko lakou naau, Ke huli nei a lele pau, Ia Iesu i pakele ae, 'Ka inaina wela e kau nei.

5 He naauao ko lakou nei, He pomaikai ke loaa mai ;

E hoohalike me lakou, $\mathrm{Na}$ lawe hala nei a pau.

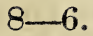

Ka hauoli ana o ka poe pono.

1 A HULI mai na hoomalau, Na hoomaloka pu, Alaila ala $a$ ulu hou, Ke oli o kakou.

2 Hauoli nui na kiai, Na kauwa a Iesu, 
Ka poe kuli, paakiki, Ke huli mai lakou.

3 Hauoli pu na hoahanau,

A mele ia Iesu,

No kona haawi ana mai,

'Ke ola no lakou.

4 Hauoli hoi, a hoomaikai,

Ko luna poe a pau ;

Ka poe huli mai no nae,

E oli oi latsou.

160.

8.

He wa maikai keia.

1 O KEIA wa, he wa maikai,

He wa e lokomaikai ai,

Kokoke nae ka po ana'e,

A pau na hana e ola'i.

2 I keia wa, hea mai lesu,

He leo oluolu no,

E huli koke mai oukou,

Ke kali nei ke $A$ kua mau.

3 Aole emo, kii mai no,

Ka make e kai ia oukou,

Imua ona e ku ai,

Malaila e hoopaiia'i.

4 A hewa no, e haule ae,

I kahi pouli ano e,

Malaila no e pau loa'i,

Na Sabati, na halawai. 


\section{PULE HOOMAU.}

5 Aole hea hou ia mai,

E mihi i pomaikai ai,

Auhea oukou, e wiki e,

O pau ka wa e pono nei.

161.

\section{8.}

E ala, a pule hóoki ole.

1 E ALA, e na hoahanau,

E ala, a e hana mau;

E pau ka hiamoe nei,

Ka manaka, ke kuihe.

2 E pau ka ohumu o kakou,

Kaumaha, eha, kanikau;

Mai hele me ka hookulou,

A kolo ae malalo no.

3 Ala'e, he hana nani nei,

Ke mele me ka hoomaikai,

$\mathrm{Na}$ leo me na uhane pu,

Hoolea nui ia Iesu.

4 E ala no i keia wa, Ua hiki mai ka Uhane laa,

E noho pu, e alakai,

Hoala, a hoohuli mai.

5 E wehe ae i na naau,

I komo mai a launa pu,

I mau ka huli ana mai,

1 mau ko kakou pomaikai.

6 E piha ko kakou naau, 
I ko ka Uhane hua mau,

Ke aloha, oli, lele pau,

Ka malu i na wa a pau.

7 Ala'e, i keia wa maikai,

Ka wa e hana aku ai ;

E ala, pule, hana mau,

I ola pu ko keia ao.

Bapetizo ana.

162.

$$
\text { 8-7. }
$$

No ka bapetizo ana.

1 MA ka wai o Ioredane,

Bapetizoia Iesu;

Hole ia a hea mai la,

"Hele mai na mea a pau."

2 "Mihi oukou i ka hala,

Huli mai e ike ia'u,

Lohe io, a paulele,

I loaa ke ola mau."

3 O ka poe i lohe pono,

Lilo lakou no Iesu,

Nana no lakou i kala,

Nana i hoomalu mau.

4 A i kona ku hou ana,

Mai ka lua kupapau,

Kena mai i na haumana

"E kaahele i ke ao.

5 "Hai oukou i kuu olelo, 


\section{BAPETIZO ANA.}

I na kanaka a pau ;

A i lohe, bapetizo,

I paulele, ola mau."

5 Ho mai, e ke'Lii Mesia,

Ho mai ia makou i pau,

I ka bapetizoia,

Me ka Uhane maikai ou.

163.

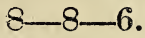

Ka launa ana mai o ka malihim.

1 E NA ohua pomaikai,

No ka Mesia e ola'i,

Ka poe i hanau hou;

He malihini nele wau,

Kuu hele hewa nae, ua pan,

E hahai me oukou.

2 Ua imi au i o, ia nei,

I kuu mau me' e waiwai ai,

Da huli naaupo:

Auhea kuu waiwai oia mau?

Ua koho e ma keia ao,

A noho nele no.

3 A lohe lea ia Iesu,

Ke kumu o ke ola mau,

Ka $i$ make no kakou:

Malaila wau i huli ai,

I ko kuu $u$ hane pomaikai,

A maha koke no. 
4 Kuu mea keia e maha'

Na Iesu wau i imi mai,

I kahi make no;

He malihini nele au,

A ia lesu kuu waiwai mau,

Aole nele hou.

5. Me oukou pu, e noho au;

No'u ko oukou Akua mau,

A me na hoa hou,

$A$ hele kana huakai,

Malaila wau e hele ai,

I hope like no.

E bapetizo ana me ka hordik

1. POMAIKAT au i keia la,

Ua pau ke kanalua o'u,

O kuu manao ua onipaa,

E lilo loa no Iesu.

2 I keia la, ua koho au,

Ia Iesu Kristo ka Mohai,

I malumalu io no'u,

I kalahala e ola'i.

3 E nana mai, ko keia ao,

$\mathrm{Na}$ anela mailuna mai,

Ke hoomakaukau nei no was,

E hui me ka poe maikai.

$4 \mathrm{Ka}$ bapetizoia mai, 


\section{BAPETIZO ANA.}

Ka hoailona e paa ai,

Ko'u ae, me ko'u hoohiki mau,

E lilo loa no Iesu.

5 Nou, ka Makua maikai o’u,

Ke keiki me ka Uhane pu,

Ke Kahi kolu oia mau,

Nou loa, kuu kino, kuu naau.

165. Pakolu ka jomaikai.

1 KE Akua, ko'u Alii maikai,

Ke ku nei au imua ou,

E bapetizoia mai,

Iloko o kou inoa mau.

2 Pakolu no ko'u pomaikai,

Pau kuu kaumaha, kuu luuluu,

Hauoli au a mele ae,

Ua pau kuu hewa ia Iesu.

3 Ua hemo au mailoko mai,

O na pahele o ka po,

Ke ku nei au e komo ae,

Iloko o kou aupuni mau.

4 Aole au e hoi hou,

Iloko o ka malu po,

Paa kuu manao e hoomau no,

A hiki ae i kela ao. 
166.

8.

He hoailona ka wai kapipí.

$1 \mathrm{HE}$ hoailona wale no, Ka wai i kapipi i $a$ mai, Ka Uhane me ke koko pu, Malaila no e maemae ai.

2 E iho mai ka Uhane muu, E hana pu, e alakai; Ilo mai kou koko e Iesu, A nana wau e hoomaemae.

¿ Kokua mai, e lesu e, I ole au e wahahee; Iloopaa $i$ kuu kino, kuu naau, lloko o kou lima mau.

4. A make au, alaila no, E kii mai na elcle ou, E lawe ia'u e ola pu, Me na haipule o lesu.

$16 \%$.

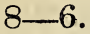

E komo ana ka poe hou.

1 NA anela, na kanaka, E nana mai oukou, Ke ku nei keia poe kauwa, E lilo no Tesu.

2 Ua paa ko lakou nei manao, Aole kuihe, E kau maluna o lakou, Ka hoailona nei. 
\$ E bapetizoia mai, Iloko o Iesu,

E hookaawale io ae, 'Na hewa o lakou.

4 E komo mai e noho pu, E hana pono mau,

$\mathrm{E}$ ahaaina me Iesu, A me na hoahanau.

5 Hauoli pu kakou a pau,

No na ohua nei,

He poe auwana, a lalau,

Ano, ua hoi mai.

6 E wehe, e na pani mau,

I komo ae lakou,

E launa, a kokua pu, 'Ke aupuni o Iesu.

168.

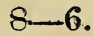

E hele mai na keiki hou.

I AUHEA oukou na keiki hou,

E hele mai oukou,

E kuikahi na naau,

E noho launa pu.

2 Hookahi kuleana nei,

Ia kakou pu maanei,

Kaumaha, luhi, pomaikai,

E loaa like mai.

3 E hoomanawanui pu, 
A pau ka launa nei, Alaila pii, a hui hou, Ma kela ao maikai.

4 Malaila no e mele mau, 'Na mele a Iesu,

Pomaikai pu, a kau, a kau,

A mau loa'ku no.

169.

\section{8.}

Ka hoailona hanau hou.

I E KE Akua, ke Lii mau,

E nana oluolu mai,

He hana nui ka makou,

O oe pu i pomaikai.

2 Ke hele nei na kauwa hou,

lloko ka hale ou,

E ku ae no imua ou,

Imua hoi o keia ao.

3 E hai i ko lakou manao,

E mihi a e lelepau,

Maluna o Iesu, a ae,

Mamuli ona e hahai.

4 Ke hoomakaukau nei makou,

E kau maluna o lakou,

'Ka hoailona hanau hou,

No na haumana a Iesu.

5 Kokua mai i pono no,

O keia hana a makou; 
Nau lakou nei aloha mai,

Nau lakou e hoopomaikai.

6 Ua pau na hana nui nei ;

Nou loa ko lakou naau,

Na kino, hana, na waiwai,

Ua lilo loa ia mai.

T Aole hiki ia lakou,

Ke hoi hou i keia ao;

E hoomau loa mamuli ou,

A pii $i$ kou wahi maha mau.

\section{0:}

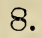

Ko Iesu aloha i na kamalii.

1 HE lokomaikai ko Iesu,

I kamalii ma keia ao,

Hoomaikai oia ia lakon,

I mai, "menei ke aupuni o'u."

2 He olioli ko kakou,

Ke lawe i na keiki hou,

E hoolaa aku ia lakou,

I mau ohua no Iesu.

3 E Iesu, e hoomaikai mai,

I ka makou mau keiki nei,

Malama, a e kiai mau,

I hele pololei lakou.

1 E ko makou Makua mau,

Hoohanau hou mai ia lakou,

I makaukau e komo pu, 'Ke aupuni nani o Iesu. 
171.

$6-8$.

$\mathrm{Na}$ kamalii liilii.

$1 \mathrm{KE}$ lawe ia nei,

Na keiki makalii,

E bapetizoia mai,

A lilo na ke'Lii.

2 Pela ke kanawai

A Iesu i kau ai,

"E ae e hele i o'u nei,

Na kamalii liilii."

3 Jesu, e nana mai

I na keiki nei,

E kau kou lima maluna'

A nau e hoomaikai.

4 Kokua ia makou,

I na makua nei,

E ao a kiai pono mau

I ka makou pokii.

5 Nau e hoomakaukau,

$\mathrm{Na}$ keiki a makou,

E hele ma kou mau aoao,

A pii ae i ke ao.

172.

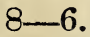

Ka berita me Aberahama.

1 E KE Akua pono mau,

Maikai ka olelo au,

Na Aberahama, kau kauwa,

Me kana mamo pu. 


\section{BAPETIZO ANA.}

2 E lilo oe no lakou, I Akua hanai mau,

A nau mai no na mea a pau,

E pono ai lakou.

3 Ua hooko pono ia mai,

Ka Olelo maikai nei,

I na makua mailaila mai,

A me na kamalii.

4 Hooiaio hoi Iesu,

'Kela olelo au,

E lawe i na keiki hou, Ma kona aupuni mau.

5 Ke $A$ kua e, ua pono mau,

Kou mau aoao a pau, Mau kou aloha ia makou,

A me na keiki pu.

$173.8-6$.

Na makua e hoolaa ana i na keiki.

1 UA lokomaikai no Iesu,

Ua hii aloha mai,

I na keiki ai waiu,

Alaila hoomaikai.

2 Mailaila mai a keia wa,

Kauoha mai Iesu,

I na makua, è hoolaa,

' $\mathrm{Na}$ keiki nana no.

3 E Iesu e, eia makou, 
Me ka makou pokii,

Ke lawe nei e hoolaa nou,

Nau i $a$ e hoomaikai.

4 Iloko o ka hewa no,

I hanauia mai,

A ma ke koko mana ou,

Malaila $e$ maemae ai.

5 Nau, e ka Uhane mana mau,

E bapetizo mai,

Hoohanau hou, i ola pu,

Me kau poe pomaikai.

17 . 8.

- Bapetizo ana o ka Uhane Hemolele.

1 KA Uhane Hemolele mau,

Mailuna māi e iho mai,

He bapetizo io kau,

He aka wale no ka wai.

2 Hoike mai $i$ kou mana mau,

E lawe, a e kapipi,

'Ke koko mana o Iesu,

Maluna o ke keiki nei.

3 E hui ka Makua mau,

Ke keiki me ka Uhane pu,

E hoopaa $i$ keia keiki hou,

I keiki na ke Akua mau. 


\section{AHAAINA À KA HAKU.}

\section{Ahaaina a ka Haku.}

Ka berena e ola'i.

1 IESU no ka Mane mau, Mane mai ka lani mai,

Ka berena na ke ao, Ka kakou e maona ai :

O ke kino pono ou,

I wawahiia mai,

Lilo mai la na makou,

I berena, e ola'i.

2 Lokomaikai kou manao,

E hoawi lilo mai,

I kou koko waiwai mau,

I mohai e malu ai ;

Awáhia ka apu ou,

No ká hewa o ke ao;

A kuu mai la no makou,

I ka waina ono mau.

3 Kahuhipa akahai,

Kei ke kolio kahe ou !

Hemolele ka mohai,

E ola'i ko keia ao,

Hanai mau mai, e Iesu,

Huikala ia makou,

Launa mai e noho pu,

I ahaaina oia mau. 
176.

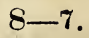

No ka ahaaina a ka Haktı.

1 MA ka puu o Kalevari,

Ka Mesia i kau ai,

A pouli mai ka lani ;

Kulou ko ke ao Mohai,

"Pau aku la," make io,

Nui ae la ka olai.

$2 \mathrm{Na}$ wahine o Iudea,

Mai $u$ we nui no Iesu,

Uwe nae, ko Ierusalema,

No ka hewa o oukou;

Mihi io, a paulele

I ka Mohai ola hou.

3 Pau aku la kona luhi,

I ka hana nui nei,

$\mathrm{Na}$ ia koko no i uhi

Ka ke ao nei mau aie;

$\mathbf{E}$ hauoli, na haipule,

Hoomanao i nei Mohai.

4 Nana keia ahaaina,

"Ka Berena o ke ao."

Kona koko no ka waina,

" $E$ inu, e na hoahanau."

$\mathrm{E}$ paulele, e na aina,

Ma ia Mohai nani mau. 
8.

No ka ahaina a ka Haku.

1 I KELA po poino e,

I lele mai ai ko ka po,

Ua hoomaau na enemi,

I pio Iesu ia lakou.

2 Nana ka berena $i$ hoomaikai,

Wawahi ae la na lakou,

Ka waina hoi, ua kuu hou mai,

Aloha ko Iesu manao.

3 "Eia kuu kino make nei, Wawahiia'e nei no oukou;

E ai me ke aloha mai ;

He ola keia no ke ao.

4 Eia kuu koko kahe ae,

I pau ka hala o ke ao;

$\mathrm{E}$ inu, e paulele mai ;

Kuu make, e hoike mai."

5 Kau ahaaina, e Iesu,

Ka makou nei e hoomanao,

A hoi mai oe, e noho pu,

$\mathrm{E}$ ahaaina oia mau.

\section{$7-6$.}

Hoomanoo in Kalevari.

1 E KE keiki hipa e, Ke noho nei makou,

Ma kau ahaaina nei, 


\section{Ia oe e hoomanao;}

E manao ia makou nei,

Kau poe kauwa akahai;

Honmanao ia Kal'vari,

A kuu aloha mai.

2 Ma kou eha ano e,

A ma ke koko ou,

Ma kou lokomaikai mai,

E kala ia makou:

Wehe i na kaula nei,

I na hewa e paa ai;

Hoomanao ia Kal'vari,

A kuu aloha mai.

3 Ma ko makou lele pau,

Ia oe ka Mohai,

Hoohauoli ia makou,

Hoopono wale mai ;

Ma kou make e pau ai,

$\mathrm{Na}$ kaumaha, luhi nei ;

Hoomanao ia Kal'vari,

A kuu aloha mai.

Ua máke eha o Iesn.

$1 \mathrm{AUWE}$ ! ua kahe wale ae,

Ke koko o Iesu ;

Ua make eha kuu Alii,

No ko'u mau hewa no. 
AHAAINA A KA HAKU.

2 He enuhe lepo kolo wau,

He kipi, hoomalau,

Ua lokomaikai mai Iesu,

A make oia no'u.

3 Ua pono ko ka la huna'e,

I kona nani mau,

A uhi mai ka pouli e,

Maluna o ke ao.

4 He pono hoi ke uhi au, 'Ko'u maka, a kulou,

Uwe, a mihi, lelepau,

A aloha ia Iesu.

5 Ma keia ahaaina au,

E Iesu ke Lii mau,

Maanei, hoohiki hou no wau,

E lilo loa nou.

180.

8.

Ahaaina hoomanao ia lesu.

1 MAANEI kakou e hoomanao,

I na kaumaha o Iesu,

Nana $i$ na nalu i kaa mai,

Ma kona uhane maemae e.

2 I kona wa i kauli'i,

Ua ala huhu kue mai,

$O$ na puali o ka po,

Me na kue o keia ao. 
3 Kokua mai ke $A$ kua mau, A lanakila no Iesu; Ma kona make eha e, Malaila no ka pomaikai.

4 Ma kona make ana mai, Malaila no e pono ai, O ko Iehova kanawai, I haihai ia ia kakou nei.

5 Nana, e Iesu, ia makou, Ma keia ahaaina au, E kala i na hewa $a$ pau, Hoola mai i ola mau.

Ua makaukau ka ahaaina.

1 MAI ke kea o Iesu,

Kani mai na leo pu,

Me na mele kamahao; "Na kaumaha, na luuluu,

Hele mai, ua makaukau $\mathrm{He}$ ahaaina no oukou."

2 Leo nani! pomaikai Kakou nei, e na pokii ; Pau ka luhi, ka luuluu, Pau ka hewa o kakou ; Hele mai, ua makaukau, $\mathrm{He}$ ahaaina no kakou.

3 Maanei no e hoomanao, 
'Kona aloha ia kakou,

Mihi, uwe, lelepau,

Me ke aloha ia Iesu,

Nana i hoomakaukau,

He ahaaina no kakou.

4 Aole emo, e pan ae,

$\mathrm{O}^{-}$na ahaaina nei ;

E hauoli nae kakou;

Mau ke aloha o Iesu,

Hea mai e pii kakou,

' $\mathrm{K} a$ ahaaina oia mau.

$18 \%$

$$
\text { 6-8. }
$$

Ka mohai e ola'i.

$1 \mathrm{HE}$ ahaaina nei,

Na Iesu, ka Mohai,

Ua make oia no kakou,

I mohai e ola'i.

2 Ke hele nei kakou,

E ahaaina pu,

E ai $i$ ke kino o Iesu,

I eha no kakou.

3 Maanei e inu ai,

'Ke koko o Iesu,

I kahe mai i kalaia'i,

Na hala o kakou.

4 E hoomanao kakou,

'Ke aloha o Iesu,

Paulele ko kakou naau, 


\section{Uwe aloha no.}

5 E haawi aku no.

I ko kakou naau,

I mohai ola no Iesu,

Ke Kalahala mau.

6 E malama kakou,

I kona kanawai,

"Kiai a noho makaukau,

No kona hiki mai."

183.

$$
\text { S-7. }
$$

He aloha kuprnaha.

1 EEHIA keia wahi!

Maanei no ka Haku mau,

E hoike pono ana, 'Kona aloha ia kakou;

He aloha kupanaha!

He mau hoaai kakou,

Ma ka papa ahaaina,

Ana i hoomakaukau.

2 Kakou no na punahele

A ke Lii i koho ai ;

Mililani, a hauoli,

Nani e ka pomaikai!

No ke aha keia pono,

I loaa mai ia kakou;

No ko Iesu koho ana,

Ia kakou e launa pu. 


\section{AHAAINA A KA HAKU. $17 \%$}

3 Nui no ka poe mawaho,

Aole lakou hele mai,

Ilihune, a pololi,

Koho nae e noho e,

Pela hoi ko kakou ano,

I ka wa i hewa ai,

Kii mai Iesu, a hoolilo,

Ia kakou i poe maikai.

4 Hoomaikai ia Iesu Kristo,

Nona ke aloha nei,

Nana kakou i hookomo,

Ma ke anaina pomaikai ;

Pule no ka poe mawaho,

E hoohuli ia lakou,

I pomaikai pu lakou la,

Ma ke aupuni @ Iesu.

Ahaaina a ka Haku.

1 MA keia papa aina au,

E Iesu ka Mohai,

Ke halawai pu nei makou,

$\mathrm{E}$ ai $i$ ka ai maikai.

2 Kou kino no ka ai maikai,

Kou koko ano e,

Ka waina i $a$ e inu ai,

Kau poe kauwa nei.

3 Ua iho mai la no Iesn.

A make no kakou, 


\section{A ahaaina pu kakou, Ma keia mohai mau.}

4 Maßnei ke kahe maikai ne

Ke kala ana mai,

A me ka maluhia e;

Me e ka pomaikai!

5) Maluna nae ka pomaikai,

I oi nui ae,

Malaila e ai pu mau ai,

Me Iesu ka Moi.

6 Kupaianaha ano e,

Ke aloha o Iesu!

Maanei makou e ae hou $a i_{\text {; }}$

E lilo loa nou.

$$
\text { Inare. }
$$

12.

$$
8-6 \text {. }
$$

No ka mare ana.

1 NA ko kakou Akua maw,

Noluna ka maikai;

- Ka oihana na kakou,

A me na kanawai.

2 Ka mare ma ke kanawai,

He hanohano no;

I paa mau âi na mare nei,

Aole hemo hou.

E hana maikai pu no hos,

I. hui na naau; 


\section{MARE.}

E imi na ka pololei,

I malu oía mau.

4 Pela mau mai ke kanawai,

I pono ai kakou;

Pela ke 'Lii e imi ai

I mau haipule hou.

5 E olioli pu oukou,

I keia pomaikai;

Mai noho" a haalele hou

I kona kanawai.

6 Pela ka mea i huli mai,

A lilo ia Iesu;

Malaila mau e hoopaa ai,

E launa oia mau.

606

8.

E mare ana laua nei.

1 E ALA kakou nei a pau,

$\mathrm{Na}$ leo pu me na naau,

Hoonani olioli e,

Ka Haku ko kakou Moi. -

$2 \mathrm{Na}$ ia la e hoolilo ae,

I na naau o laua nei,

I naau kuikahi mau,

I nononoho malu pu.

3 Wahine-kane, mare pu,

Hookahi io, ka manao, 
Ka waiwai hoi, hookahi no, Aole e kaawale hou.

4 In a pela, pomaikai no,

E hooikaika a hooko,

E like me ko laua ae,

E like me kou kanawai.

5 E Iesu e, o oe pu,

Me laua nei, e alu mau,

I lako i koonei waiwai,

Me ko ka lani pomaikai.

18\%. E 1 mare ana lakou nei.

1 KA Haku e, e iho mai,

Iwaena o makou,

Hromakaukau ia lakou nei,

I ala a noonoo.

2 He oihana nui nei,

Nau i haawi mai,

He mea hoohiki, e paa ai,

- Kokua i pomaikai.

3 E mare ana lakou nei,

E huipu i $a$ mai,

E like me ke kanawai

A Iesu i kau ai.

4 Aole e kaawale hou,

Ma keia hope ae, 
Ka make, oia wale no, Ka mea e hemo ai.

5 E noho pumehana pu, $\mathrm{E}$ hana a maikai, Ka hakaka, kue, huhu, Mai launa ia mai.

6 E Iesu, e hoopiha mai, Ko lakou nei naau,

I ke aloha, i paa ai, Ke kuikahi mau.

$$
8--6 .
$$

Ahaaina mare.

1 I KELA wa i noho ai, Iesu ma keia ao,

Ua hoomakaukau ia mai,

He ahaaina no.

2 He ahar ina mare nae,

Malaila pu Iesu,

A nana i kokua mai,

Ka nele o lakou.

3 Maaniei no hoi, e Iesu e,

E iho pono mai,

E launa me na mare nei,

Nana, a hoomaikai.

4 Hoomaopnpo ia lakou,

I keia hana e, 
O hana me ka naaupo, A hewa avanei.

5 Honpau $i$ ke ano kamalii,

I noho a kuoo,

Ma keia hope aku nei,

A hana pono mau.

6 I kela wa, i keia wa,

$\mathrm{O}$ oe ke Alakai,

Ma keia ao, a pau na la,

Nau e kokua mai.

7 A kau ka make ia lakou,

A hookaawale ae,

Hoomakaukau e hui hou,

Ma kela ao maikai.

\section{Malama Hou.}

189.

$$
\text { 7-6. } 6 \text {. }
$$

Aloha ko na aina e.

1 ALOHA ko na mauna,

I paa mau i ka hau,

A me ko Aitiopa,

Ko Inia me Makao,

$\mathrm{Na}$ muliwai kahiko,

Na moku na papu;

Kii mai kolsila pio,

I ola no lakou.

2 Auwe na lahuiaina

O na akua e! 


\section{mALAMA HOU.}

Ka MAKE O na aina

I kulou i na kii!

Ko Aferika pocur!

Ko Asia nauvo!

Ko Mahomeda PשLE?

Ko Roma hewa hou!

3 Pehea la ke hoole

Kakou i aoia mai

$I$ kanaka pouli,

Ia lama e ala'i ?-

Ke OLA! O Ke OLA

Hooluha ae kakou,

1 lohe $i$ ka Mesia,

A $e$ huli ko ke ao.

4 E lawe e na makani

I kana olelo mau;

I uhi kona nani

I ka honua a pau-

E hoi hou mai ka Alana,

I make no makou,

Ka Moi olla mana,

Ke Lij pomaikai mau.

Ka mahinaai.

] UA makaukau ka aina hou,

Ua luluia ka hua mau;

E ka Uhane $e$ ua mai,

I maikai kau mahina ai. 
2 A keokeo maikai mai

O ko Iehora kihapai,

Na no anela e ohi no

$\mathrm{Na}$ hua pono no ke ao.

191

\section{8.}

$\mathrm{Na}$ aina a pau e mele pu.

1 HOOLE $A$ na kanaka a pau,

I ke 'Lii nana $i$ hana mai ;

$\mathrm{Na}$ aina a pau e hapai pu,

I ke Akua e ola'i.

2 Mau loa kou aloha mai;

He oia mau ka olelo au;

Nou, e Iesu, ka hoomaikai,

A pau na la, a pau na po.

192.

7.

Ka Iubile.

1 MELE i ka Iubile,

E hookani ma na kai ;

Kani ma na aina $a$ pau,

'Iesu ke 'Lii ola mau.'

2 Oli pu mai o a 0 ,

Ola nui, e ko ke ao,

Hiki mai ka Iubile,

'Iesu ke 'Lii o na lii.'

3 Mele pu na anela,

'Kou aupuni ke ku paa

Nou ko lalo, nou ke ao,

E Iesu, ka Moi mau.' 


\section{MALAMA HOU.}

193.

11.

He mele kamailio.

Kanaka.

1 OWAI la ka mea e hoike mai,

I ike makou i ka mea maikai? -

Misionari.

Hoolohe oukou i ka me' pono e, He ola ka makou e hai aku nei.

Kanaka。

¿ Auhea la ka pono? e hai mai oukou, Auhea la ke ola e ola'i makou?-

Misionari.

A i luna ke $A$ kua ka Haku maikai,

No ia la na lani, na aina, na kai-

Kanaka.

3 Huhu paha ia, e maké makou;

Auhea la ke ola? e hai mai oukou.

Misionari.

Na Iehova he mau kanawai i kau mai Hoolohe mau ia, ka me' e ola'i-

Kanaka。

4 Ua haki-ua hewa-ua make makou; Auhea la ke ola? e hai mai oukou.

Misionari.

O kana Mesia ua iho mai nei-

A malama pono ia mau kanawai-

Kanaka.

5 Pehe $a$ hoi ia ia e ola'i makou?

Auhea la ke ola? e hai mai oukou. 8 * 
Misionari.

Kii mai ka Mesia i ola oukou-

Ua pepehiia, make e ia kakou-

Kanaka.

6 Auwe i ka make! ua hewa makou! Auhea la ke ola? e hai mai oukou. Misionari.

Kau ia ia ka hala o kakou a pau. I make-ua ola-ua hoi i ke ao-

Kanaka.

7 Pehea la ke ano? e hai pono mai; Mahia la ka hewa e kalaia'i ?-

Misionari.

Mai kanalua'e i ka Mohai maikai, E paulele ia ia-e ola mau ai.

Kanaka.

\& Oiaio ke ola!-Pomaikai makou! Me e ka Mesia e ola'i kakou :-

\section{Pau pu.}

Ua ikea ke ola ! mahalo ke ao! la Iesu ke aupuni aole e pau.

$6--4$.

1 HE luaole hoi,

$\mathrm{Na}$ aina o ke kai!

Ke pae nani nei,

Ka leo hou maikai,

$\mathrm{Ka}$ otelo e hauoli ai,

'Ua pau na kii akua nei 


\section{MALAMA HOU.}

2 Na akua wahahee, I puni ai na lii, $\mathrm{Na}$ kapu ino nei,

I make nui ai;

Ua hooleiia, ua lilo e, Ua pau na kii akua nei.

3 Iesu ke 'Lii maikai, Ka Lanakila e, Kou lima i pau ai, Na akua wahahee,

Hapai makou i kou maikai,

Ua pau na kii i make ai.

4 lehova oía mau, Ke Akua e ola'i, Ke kali nei makou,

I kona kanawai;

Na kona mana e kinai,

Na kii o ko na aina e.

$5 \mathrm{Na}$ Misionari e, E hai $i$ ke ola mau, Iesu e honmaikai, I huli ko ke ao;

I lilo pu i hale nou,

A mele $i$ kou aloha mau. Lokahi ka manao, 
E pule $i$ ke Akua mau, I ola ko ke ao.

2 Mamua, noho naaupo,

$\mathrm{Na}$ moku nei a pau,

A puka mai ka la maikai,

I naauao kakou.

3 E ko ke ao nei Haku mau,

E lokomaikai mai,

I holo ma na ain $\alpha$ a pau,

Ka olelo e ola'i.

4 E hiki mai ke aupuni ou,

A puni keia ao,

I lohe kanaka a pau,

A malu mai lakou.

196.

$$
\text { 8--7. }
$$

No ka holo ana o ka euanelia.

1 MA na mauna pouli wale,

Nana kuu uhane e!

Wehe mai ke alaula nani,

Eia mai ka Iubile;

Oraio!

Ko Iehova nani nei!

2 E hoike ma Asia, Me ko Aferika po,

I ko Iesu lanakila,

Ma ko Kalevari puu,

I hoolana,

Ko na aina welelau. 


\section{MALAMA HOU.}

3 Na aupuni noho pouli,

Nau no e hoonaauao,

Mai hikina a komohana,

Pale kolse i ka po,

I ao maoli,

I ikea ka nani ou.

4 Hólo; e ka Euanelio,

Holo lanakila mau,

Nou e lilo ai na pio

I ohua no Iesu;

E ka Haku,

Nou na aupuni o ke ao.

$19 \%$.

$$
8-6 .
$$

No ka poe ola ana iuka.

$1 \mathrm{E} \mathrm{KO}$ makou Akua e,

Ke Alakai maikıi,

$\mathrm{Na}$ kou aloha makou nei,

I hoopae ola mai.

2 Nou mai la ka makani-nou-

Ooloku ke kai ;

A holo pomaikai makou,

Me oe ke Alakai.

3 Ua kiai lokomaikai mai

$\mathrm{Na}$ maka ala ou ;

Nou i malie ai ke kai,

Ka ino hoi ua pau.

4 Kou lima mana, e Iesu,

Ka me' pakele ai; 
Nou hoi ko makou mohai hou,

Ka mohai hoomaikai.

5 A holo lua makou nei,

I ka moana mau,

E alakai, i loaa mai

Ke awa o ke ao.

$190.7-6$.

Na kanaka a me na anela e hapai pu.

1 NA ksuwa a ke Akua,

E hai i ka Moi,

I puni ka honua,

I kona nani e:

Ka inoa lanakila,

O Iesu ke hapai,

He aupuni hiwahiwa,

Ko ka Mesia nei.

2 Maluna ko ke Akua,

Hoomalu ana mai;

$\mathrm{Na}$ lesu i kokua,

Ka ekalesia nei :

$\mathrm{Na}$ ke anaina ona,

E hoomaikai ke Alii,

No Iesu mau ke ola,

No ko kakou Mri.

3 Ke ola i ke Aliua,

Ka Lunakanawai;

Hooho ka honua,

I ko ke Lii maikai; 


\section{MALAMA HOU.}

Ko Iesu mea mahalo,

Na na anela e hai;

Ua moe lakou ilalo,

Hoomana $i$ nei Mohai.

4 Kakou ke hookaulana,

I ko Iesu maikai,

Ka nani me ka mana,

Ke ao, ke akamai,

$\mathrm{Ka}$ ihi, ka mahalo,

Me na anela a pau,

E hai ma kona alo,

I ke aloha mau.

199.

$$
8-6 .
$$

No ke aupuni o ka Nesia.

1 POMAIKAI wale ko ke ao!

Ua hiki mai ke Alii,

E moe, e na aina $a$ pau,

E malu nui mai.

2 Hauoli! O Iesu ke Alii!

Mahalo kanaka;

Hookani ae na mea a pau,

E pau hoi na kina.

3 Mai ulu wale hou maanei,

Na ino no ka po;

$\mathrm{Na}$ Iesu i hoawi mai

Ka maikai wale no.

4 A nana e hoomalu mau,

$\mathrm{Ke}$ aupuni pomaikai, 
A ike pu na aina $a$ pau,

Ia pono e ola'i.

200.

$$
7-6 .
$$

Na kiai o Ziona.

1 E NA kiai o Iesu;

Mai makau, a hakanu;

E hookani i ka pu,

Hoonui, a hoomau :

Hele nui ae oukou;

Hai i ke kauoha hou;

Holo hoi mai o a o,

A huli keia ao.

2 Ma na mauna, ma na kai,

Ma na kapa muliwai ;

Hai i ka Logou maikai,

I kanaka a pau;

Ina hoomaau lakou,

Mai pauaho; ku paa no ;

Mau me oukou ka Logou,

A pau ae keia ao.

3 Aia ke lii o ka po,

Me na kini nui no,

Ku e i ke $A$ kua mau,

A kaua hewa mai ;

Ma ke aloha ia Iesu,

Mai haalele; alu pu;

Kii, hoohuli $i$ ko ke ao,

A maha pomaikai. 


\section{MALAMA HOU.}

4 Eu na hoalunahai,

Aia hoi! ua lilo mai!

Nani keia huakai!

Ua lilo ia Iesu :

Aia pau ka paio nei,

Kani lanakila e,

Mele $i$ ke Lii e ola'i,

A noho malu pu.

$$
8-6 .
$$

Ko Iesu hoomalu ana.

1. KAKOO, e ke Lii mana e,

I pahikana nau,

E o i kou mau enemi,

I lohe mai lakeu.

2 F holo lanakila ae, Me kau olelo mau;

Kau pahikaua mana nei,

$\mathrm{E}$ malu ai ke ao.

3 He nani kou aupuni mau,

Me e ka pomaikai ;

E malu na haipule au,

I kou hoomalu mai.

$4 \mathrm{E}$ holo me kou nani nae,

Ma kou kaa ola mau,

E hole weliweli ac,

Nou loa keia ao. 
1 NANI na wawae n lakou,

I lawe mai i lia olelo hou,

I hai mai hoi ia me' maikai,

I malu ai na aina nei.

2 Ire mea mahalo nui nei ;

O ko Iehova kanáwai;

Hauoli pu mai o a o,

Na makamaka o kakou.

3 Ua aloha wale mai lakou,

I keia aina naaupo;

I hoouka mai $i$ ka waiwai mau,

No na uhane o kakou.

$4 \mathrm{Ka}$ poe $\mathrm{i}$ noho hune no,

Aloha nui ia Iesu,

I haawi lokomaikai mai,

Ia waiwai maoli e ola'i.

5 He palapala pololei,

Ile olelo pono e ola'i ;

E huli nui no kakou,

Ma ka Iehova pono mau

6 E hele ma ka pololei, Akahi no ka pomaikai;

No lesu ke aloha mau,

Nona ke ola o kakou. 


\section{MALAMA HOU.}

\section{3. $8-5$,}

Hazoli no ka lolie ana i na luna hai ola.

1 HE nani no na kapuwai,

Ma ko Ziona puu, .

Ke ku nei e hoike mai,

He ola no kakou.

2 He leo lea ko lakou,

He olélo nani e;

'Ziona, eia ke 'Lii ou,

Ke lanakila nei.'

3 Pomaikai no na pepeiao,

I keia leo hou;

Na maka nei, pomaikai pu,

I keia nani mau.

4 Hoonani mai nei na kiai,

Memele lea no;

Hapai Ierusalema nei,

Hosana ko ke aø.

5 Na Iesu e hoomalu no,

A malu keia ao;

E ko ke ao, e ike pu,

Iesu ke Ola mau.

201.

Aloha i ko na aima naaupo.

1 NA miliona nui no,

O ko na aina naaupo,

Aole no i lohe mai,

I ka Mesia e ola'i. 
2 Hoouna, e Iehova e,

I holo kau olelo nei,

I me' e nani koke ai,

E like ne ka la laelae.

3 Kokua i na luna hai

I aoao e pakele ai,

O iho i ka make mau

Ka poe hoomanakii a pau.

4 Paipai i na ohua ou,

I lokomaikai mau lakou;

$\mathrm{Na}$ kino, me na waiwai pu,

Hoano lilo no Iesu.

5 I nui na paahana hou,

E hele me ka Uhane ou,

A mele nui keia ao

I kou Hoola kamahao.

205. 8.

O Iesu ka Hoku no ko na aina e.

1 E ALA, e na moku e, Ke moe ma ka malu po, Nana oukou, ua puka mai,

Ka maamaama no oukou.

2 Ke lawe aku nei na kai,

I. ka ukana maikai ae,

E na makani, e nou mai,

I pae ka waiwai e ola'i.

3. Pomaikai ko na aina e,

I noho ma lia malu po, 


\section{MALAMA HOU.}

Ua poha mai ke ao maikai,

lesu ka Hoku no lakou,

4 E pau na kii, me na heiau,

E pau na mohai naaupo,

Iehova ke Akua mau,

Iesu ka Mohai no lakou.

5 Hoomana e na aina a pau,

I ke Akua ola mau,

Hahai mamuli o Iesu,

I pii oukou a noho pu.

\section{7.}

E na kiai, heaha ke ano o ka po?

1 E NA kiai, e hai mai

I ke ano o ka po,

E ka malihini nei,

Nana i ka Hoku mau,

Hoku aha? e hai mai ;

Hoku hoomaikai anei?

Hoku lilelile no,

No na hanai a Iesu.

2 E na kiai, e hai mai,

I ke ano o ka po ;

E ka malihini nei,

Ke pii ae ia Hoku mau

Nui anei ka maikai?

Malu, maamaama anei?

Poha kona lama hao,

Ma ko keia ao a pau. 
3 E na kiai, e hai mai,

I ke ano o ka po;

E ka malihini nei,

Ke hee alsu nei ka po.

Pau ka huli io ia nei,

Hoi i ka malu ou;

Eia hoi, ua puka mai

Iesu, ke Lii malu mau.

$20 \%$

$8-4$.

Ka Hoku mau.

1 F KE 'Lii o na a a pau,

Hoolaha i lou nani mau;

I keia wa, hoopuka mai,

Ma kou Ziona pomaikai,

Ka Hoku loa e ola'i,

Ka Hoku mau.

2 Kokua a hoonaauao,

I ko na aina naaupo,

$\mathrm{Na}$ aina paa malalo ae

O ko Iehova kanawai,

Ke kanawai e make ai;

Ka make mau.

3 Iesu, e hookahuli ae,

I hookahuliia mai

$\mathrm{Na}$ aina e mai o a o,

E iho ana i ka po,

I huli i aupuni nou,

Ka Haku mau. 
$4 \mathrm{Ma}$ kau olelo mana mau,

E huli ai ko keia ao;

Malaila no e pau loa'i

Na 'kua kii, na mea kue,

A hoomana'i na aina e,

Ke Akua mau.

208. 6--8.

He halawai pule i ko na aina e.

1 ALOHA kakou nei

Na aina naaupo,

Ke hele nei e pule ae

I ola no lakou.

2 Ke pae nui mai,

Ka leo no lakou,

Ua puni i ka wahahes,

A me ka malu po.

3 Ke hea mai lakou,

'Nana aloha mai,

E lawe mai $i$ ka pono mav,

Ka pono e ola'i.'

4 Nolaila no kakou,

I hui aj maanei,

E pule, a e hoomanao,

I ko na aina e.

5 E pule mau kakou,

E lokomaikai ae, 
Kokua, a makana no,

I ko kakou waiwai.

6 Pela e pono ai,

A oluolu no,

Iesu, me ko na aina e,

A huli mai lakou.

2097.

E hiki koke mai ko Iesu aupuni,

1 F KA Haku mana e,

E hoohiki wawe mai,

I ka wa e huli ai,

Ko na aina, ko na kai,

Na me $a$ nui, na liilii,

Na kanaka, me na lii ;

E pau no lakou a pau,

I ka lilo no Iesu.

$2 \mathrm{Na}$ aupuni naauao,

Me na aina naaupo,

E hoolohe ia Iesu,

Huli mai a ola pu.

Pau ka mana o ka po,

Paa Satana i ka hao,

Lilo keia ao a pau,

I aupuni no Iesu.

3 Pau na kaua, na kue, Me na haunaelè nei,

Kahe ae ka pomaikai,

Ma na aina, ma na kai 
Lanakila wale ae,

Ko Iehova kanawai ;

Maluhi $a$ na aina $a$ pau,

E hoomalu mau Iesu.

210.

8.

No ko na aina e.

$1 \mathrm{E}$ KO Iehova lima e,

Ala'e, ala'e, hoike mai,

Ka mana ou e huli ai,

O ko na aina, ko na kai.

2 Hoike i ko keia ao,

Kou lanakila ana mai,

Ma kau olelo maikai pu,

Me kou aloha e ola'i.

3 Hoike i na aina e,

'Iehova ke Akua mau,'

Ma kona leo e pau ai,

$\mathrm{Na}$ lele, kii, me na heiau.

4 Hoohiki mai $i$ ka wa maikai,

$\mathrm{E}$ hoihoi pu mai ai,

Iloko o kou kihapai,

Na ludaio, me na aina e.

5 E holo oe mai o a o,

Ka lokomaikai nui ou,

I huli mai na aina $a$ pau,

A lilo i aupuni nou. 


\section{IIIE $\mathbb{N} \mathbb{I}$.}

211. Kokoke mai ka wa maiká

1 E KOKOKE io ana

Na manawa ano hou,

E kokua mana ana,

Ke Akua mai o a o;

E pau ana,

to ke ao nei naaupo.

2 Keia wa, ua paapu wale

I na ino, na kue,

Aka, e makaukau ana

Na mea mana e pau ai;

Na Iehova,

E hoolanakila mai.

3 Iriki pinepine mai $\mathrm{la}_{3}$

O ka lohe ia kakou,

No ka huli nui ana,

Ma na aina naaupo;

Wehe ae la,

Ko lukou la wanaao.

4 Ko Lalioba Haku mana,

E hoike ia makou,

I La lanakila ana

O ka olelo nani au,

Ma na aina,

Ma ko keia ao a pau. 


\section{MALAMA HOU.}

$$
\text { 8-7. }
$$

No ka poe hoomana kii.

I MA na aina pouli wale,

E nana aloha nei,

Ike ole, a hoomana

I na 'kua wahahee;

Kini, lehu, miliona,

Ke poho la i ka po.

2 Iesu, e ka Hoku loa,

Lama no ko keia ao,

E hoopuka $i$ kou kukuna

Ma na aina naaupo,

I ikea kou mamaama,

E na maka o lakou.

3 Kela poe e kulou ana,

Ma na kii kala, laau,

E kii oe, a hoohuli

Ia lakou mainuli ou,

E hoomana ia Iehova,

I Akua no lakou.

4 E Iehova, nou ka mana,

E ko ai na mea maikai,

$\mathrm{E}$ hoouna $i$ na kahuna,

$\mathrm{Na}$ lakou e hoolaha'e,

Kau olelo, kou aloha,

$I$ ola ko na aina e.

1 NA ke aha e hoohuli, 
I na aina naaupo?

$\mathrm{Na}$ ka Uhane Hemolele,

Kona mana wale no,

E ka Uhane,

E hoohuli ia lakou.

2 Na Kahuna hai olelo, Make hewa a poho, Ka lakou la hana luhi,

I ole oe e hana pu;

E kokua,

I na hana a lakou.

3 Na na Kaula i hoike,

- Na manawa pomaikai,

Ma na la mahope aku;

$E$ hoohiki wawe mai;

E ka Uhane,

Ma kou mana e ko ai.

4 Ina aua, e ka Uhane,

I kou mana, e poho Na manao, na pule, hana,

No na aina naaupo, A palemo,

Lakou i ka make mau.

$\mathbf{E}$ hele, $\mathrm{j}$ ko na aina $\mathrm{e}$.

1 E KO Iehova poe kauwa, E hele ae, mai o a o, 
Aloha i na aina paa

Iloko o ka naaupo.

2 Malaila no e noho ai,

Mawaena o ka poe kulou,

A hoomaikai $i$ na'kua e,

E iho ana i ka po.

3 He aina waonahele no,

Ke uhi la ka malu po;

Malaila e kukulu ai,

Ko Iesu kea e ola'i.

4 No Iesu no na aina $a$ pau,

$E$ hele e hoohuli mai,

E hoohaumana ia laknu,

Na Iesu ke 'Lii e ola'i.

5 E hele pu no hoi Iesu,

E alakai, e kiai mau,

Ka Uhane hoi e ala pu,

A paa ka hana a oukou.

$$
7-6 .
$$

E hele e boike i ka mea e ola $\mathrm{i}$.

1 NA elele a Iesu,

$E$ ala, a e hele pu

I na aina naaupo,

E hai $i$ ke ola mau ;

Hele me ka lelepau,

Ma ka Luna o oukou,

Luna lanakila mau,

Iesu ke Kamahao. 
2 E kukulu ae oukou, I ke aupuni o Iesu, Ma na aina i paapu, $\mathrm{Na}$ 'kua wahahee; Lapuwale ko lakou, Hana ino wale no, Ke iho la i ka po, Auwe! ka make e!

3 No Iesu na aina e, Nani kona aloha mai, Nake oia, e loaa'i Ke ola ia lakou; Hele e hoike ae, Ke aloha e ola'i, Kela poe hoomanakii, I ola ia Iesu.

4 Paio oukou, paio man, Ma ka pule, ma ke ao, I auhee ka malu po, A ike ae lakou; $\mathrm{Na}$ lesu e hana pu, $E$ w?wahi $i$ na heiau, Me na 'kua kii a pau, E lana ka manao.

Hoolaa ana $i$ ka luakini. 8. Hoolaa ana $i$ ka hale pule. 1 E KE Akua mana mau, 


\section{MOOLAA I KA LUAKINI. $20 \%$}

Ke makemake nei makou,

E lilo keia hale hou,

I luakini kapu nou.

2 Mai luna mai e tho mâे, A komo mai maloko nei, Me makou nei e noho ai, Kokua mai a hoomaikai.

3 A pule aku makou nei, Hoolohe a haawi mui; A mihi ko makou naau, Ko makou bewa, e hoopau.

A A ku e hai kau poe kauwa,

I kau olelo, ma kou la,

O oe pu, kokua mai,

I huli nui na kue.

5 E Iesu, me ka Uhane ou,

E iho mai a launa pu,

I lilo keia hale hou,

1 luakini maenae mau.

1 E IHO mai anei Iesu, Mai kona noho moi mau, F kome mai, e launa pu, Ma keia luakini hou?

2 He lokomaikai ano e! Hoonani aku kakou nei? 
Ke ae nei ko kakou Ali,

$\mathbf{E}$ hele a kokoke mai.

3 Nou keia luakini hou,

E piha i kou nani mau;

E kani mau maloke nei,

Ke mele me ka hoomaikaí.

4 Naanei e alu pu mau ai, Kou mana i na luna hai,

I huli mai na hoomalau,

A lana ko makou manao.

5 A hiki mai kou la hoopai,

Alaila e akaka mai,

Ka poe i huli mai maanes,

Lakou ke ola pomaikai.

6 Akaka hoi ka poe kue,

A mau ko lakou paakiki;

Mai luna, mai kou alo mau,

E iho lakou i ka po.

\section{Kakahiaka.}

Himeni kakahiaka.

1 UA wehe mai ke alaula hou,

Ua hala e ka malu po,

Ke hea aku nei makou,

Ia oe, e ke Akua mau.

2 Ko mau ehers i whi mas

Ia makou nei i moe ai ; 
Ula trià mai na maka ou,

O meke makou i ka po.

虂 he mai i ka Uhane ou.

1 Kumu ao mai ia makou,

I hahai makou nei a pau,

I hana pono madi no.

- A bele aku makou nei,

E lawehana e hana'i,

Ealakai ka mana ou,

I hewa ote ai makou.

5 E imi makou ia Iesu,

E noonoo nra ke kanawai,

I liu paa ho makou manas

I kana pono e ola'i.

Ahiali.

Himeni ahiah.

1. UA hala keia la,

Ua uhi mai ka po:

Pela e malu koke mal

Ka make o kakou.

2 Lra i makæukau

E maha me Iesu,

He maluhia ko kakou.

Ke nalo keтa ao.

3 Ua pomaikai kakou,

Ua launa mái Iesu. 
A ao ka po a po ke ao, He ahonu: no.

4 Hoomalu ia makou, E lesu, i ka po;

E kiai mai na anela, A hiki mai ke ao.

5 A ike makou nei,

I kou la nani no,

E holo pu i loaa mai Kou nani i ke ao.

6 A po ko makou la, Ke pule nei makou,

E maha ma ka poli mau

O ki aloha ou.

Hoomaikai ana.

$2010 . \quad 8$.

Himeni hoomaikai i ke Akua.

1 KE Akua lokomaikai mau, E ku makou imua ou, E mele me ka hoomaikai la oe ko makou Alii.

2 Ua mau ka lokomaikai ou; Ke hooloihi mai nei no, Ko makou noho ana mai, A me ko makou pomaikai.

3 Ua hoomauia ka launa mai O na makua, na kamalii, 
Na kumu, na haumana pu, Na kahu, me na hoahanau.

霍 $\mathrm{Na}$ makainana me na'lii, Va hoopomaikai pu ia mai;

Na mea a pau e pono ai,

Ua heawi wale ia mai.

5 Ua hoomania ia kakou Na kino me na uhane pu, Na kula me na halawai, Na mea e naqua mai ai;

6 Na mea no hoi e pono ai Na moku o ke aupuni nei, Ke kuikahi e paa ai Ka malu me na aina e.

- Koonani kakou nei a pau, Na leo pu ne na naau, I ke 'Lii nui o kakou, No kona lokomaikai mau.

$$
8-6 .
$$

E hoonani kakeu i ka Haku.

1 HOONANI e na aina nei, Na kanaka a pau, Hornani kn kakou Aji, No kona aloha mau.

2 O lesu ko kakou Alii; Ma kona mana mau, 
E. malu ai na aina ne A pomaikai kakou.

3. Havoli e na moku nei

Na mauna, na papu,

E hoomakauka ia ma

I mele no Iesu.

4 Na kona loknonaikai mat:

I. haawi wale mai, Ke ola, malu, nanuao, Me ko kalsou waiwai

5 Namea a par e pono an

Ko kekou ola nei,

A me ke oln maluna'e,

Malala e loaa'i.

6 E.mau kou k kmaika mas E. Iesu ke Alii, I malu mau ke ola nei.

A ola maluna'e.

8.

E mole i ka mea e ola ${ }^{\%}$.

1 E MLLE aku kakou ne,

Hookiekie nui ae

I ko kakou nei hoomaikai,

I ke 'Lii nani e ola'i.

2 Ke Akua ke 'Lii mana mau, Oia ke Kahu no kakou, 
$\mathrm{Na}$ ia la $\mathrm{i}$ hoawi mai Ka hanu, ola, pomaikai.

3 Hoolohe kakou nei a paw, I kona leo hea mau, E ae i kona kanawai, Mahalo me ka hoomaikai.

4 Wai hoohalike me lakou, Ke poe kipi, hoomalau, A hoopaina a poho Halo lilo i ka po.

5 Ina kakou, e naue ae, I ko Ziona puu maikai; Paulele $i$ malu ai kakor, A komo i ka maha mar.

1 E MELE kakou nei, Ma keia la makai,

He la hauoli no kakou;

E mele ia lesu,

Ko kakou Haku mau,

$\mathrm{Na}$ leo pu me na naau.

2 Ua kißi mai kela, la kakou na kauwa, A po ke ao, a a ka po, Ua mau ka noho $\mathrm{pu}$,

I malu ai kakou;

Ifoonani nui ae kakon. 
3 lwaena konu no

O ko kakou poho,

Kii mai kela, hoala mai ;

Ua pale nui ae

Na eha, ino, mai ;

Hauoli me ka hoomaikai.

4 Kakou na hoahanau,

Ua maluhia mau,

Aole $i$ hoomaauia mai;

Kokua mai na'lii,

Auhee na enemi;

Nani ko kakou pomaikai.

5 Ua haawi ia mai

$\mathrm{Na}$ mea e pono ai

$\mathrm{Na}$ kino make o kakou;

Ua mau na Sabati,

Na kula, halawai,

Mailaila mai ka pono mau.

6 E ala nui mai,

Na leo hoomaikai,

Ma keia la hauoli no;

Hiilani ia Iesu,

Ke Lii hoomalu mau,

Ia kakou me na mea a pau.

Hope o ka makahiki.

1 HEAHA la kakou? 


\section{MAKAHIKI HOU.}

Ko kakou ola nei ?

He maha pua iki no,

A nalo koke ae.

2 Auwe! ke lele nei,

Na hora o kakou,

E like me ka wai paihi,

Pela ka lele mau.

3 Aole emo, pau

Na makahiki nei;

E noho me ka hana mau,

Na hana e ola'i.

4 Ke bolo nei kakou,

Maluna o ke kai,

Ke kai kupikipikio.

1 kahi e pae ai.

5 E lana ka manao,

Kokoke no e pae,

Ke awa o ka lani mau,

Ka maha pomaikai.

Makahilki Hou.

205.

Makahiki Hou.

1 NA makahiki o kakou,

Ke holo kiki nei ;

Eia'e ka makahiki hou,

Hauoli, hoomaikai.

2 Hauoli, a e noonoo nae,

Paapu na mea kue, 
E makaala, e kiai, E hele pulolei.

\& Maluna o ka wa ano

Ke kau na mea mau, Ke ola me ka make pu,

E naauao kakou.

4 E Iesu e, e paipai mai, Ia makou e ala'e,

E make paha $a$ hele ae, Ka makahiki nei.

5 A pau na makahiki nei, I hea e noho ai?

lluna i ke ao maikai? Ka lua po anei?

926. 7.

1 HOLO naha ole no, O ka makahiki $i$ pau; Nui no na hoahanau, Pau ke ola o lakou; Aole halawai pu hou, Me kakou malalo nei, Paa mau no ma kela ao, Aole laliou hoi hou mai.

2 Kakou nae ke ike nei Keia makahiki hou,

E lesu, e halawai Me makou a noho pu; 


\section{MAKAHIKI HOU.}

E kokua, e hoomau

I ka lokomaikai ou;

Ahonui no, a pau

Keia makahiki hou.

3 Nani kou aloha mai,

I na la i hala ae nei;

Pono no ma keia la,

E noonoo, a e nana

I na hana maikai au,

A hoonani na naau;

E hauoli ae kakou,

Ma ka maliahiki hou.

4 Pono hoi ke hoomanao,

Ma ka makahiki nei,

Kii mai paha o Iesu

Ia kakou e hele ae ;

Pomaikai ke makaukau

E haalele $i$ keia ao,

A pii aku iluna'e,

Me Iesu e noho ai.

$22 \%$

$$
\text { 8--6. }
$$

Makahiki Hou.

1 E KUU Uhane, e noonoo,

Ua hala iho nei

Kekahi makahiki hou,

O kou wa e ola'i.

2 E hoomanao, aole mau

Na la o keia ao; 
Ma keia makahiki hou,

Malaila paha pau.

3 Nui na makahiki ou

I hala aku nei,

Na mea e koe, pau koke no,

Ke holo kiki e.

4 E ala, e kuu whane e,

Me kou ikaika a pau ;

$\mathrm{E}$ imi i kou ano nei,

Heaha kou manao?

5 Ma keia la ua puka mai

Ka makahiki hou,

Ano no oe e hoomaka'i,

E pii $i$ ke ola mau.

228.

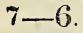

Makahiki Hou.

1 E LAWE aku ana

$\mathrm{Na}$ makahiki hou,

I ko kakou uhane

I kona wahi mau;

Pokole koonei ola,

He la hooilo no,

He hele wale ana

Ka lua kupapau.

2 Ka wa opiopio,

Ka ikaika o kakou,

Ka nani o ke kino,

Ka waiwai o ke an, 


\section{MAKAHIKI HOU.}

E lilo aku ana,

Ka wa e make ai,

A hele na uhane,

$\mathrm{E}$ hoopai ia mai.

3 Na makamaka aloha,

E hoomanao kakou,

Pau koke koonei ola,

Kokoke kela ao.

Ma keia makahiki,

E make ana no,

Kekahi onei kini,

Kekahi o kakou.

4 Owai la? aole ike,

Aole no kakou

Ia wahi pohihihi,

No ke Akua no;

O kana mau kauoha,

Ua ike nae kakou,

"Kuoo, a mau ka pule,

E noho makaukau."

Makahiki Hou.

1 ALA, ala, e nana,

E pan ana no na la,

Puka emo ole no,

Keia makahiki hou;

Nani ko Iesu maikai,

Kona ahonui mai ; 
Mililani kakou nei, E hoolea, hoomaikai.

$2 \mathrm{Na}$ makua, kamalii, Na kanaka, me na'lii, $\mathrm{Na}$ kahuna, kumu ao, $\mathrm{Na}$ haumana, hoahanau, Konohiki, na lopa, O na luna, na haahaa, Na kahiko me na hou, Me koonei mau mea a pau;

3 Nani e ka pomaikai, No ka hiki ana mai

O ka makahiki hou, Aloha kakou nei a pau;

E hoohiki hou kakou, Keia makahiki hou, E hahai $i$ na la a pau, Ia Iehova ke Lii mau.

\section{Kupapar}

230. 8

Ka make ana o ko keia ao:

1 KO keia ao, he lepo no, E hoi ilaila no lakou, Na mea nui, na me' linlii, Ma na ilina $e$ moe ai.

2 E hoomanao maikai kakou, He mea weliweli nei, 


\section{KUPAPAU.}

E huli koke pu ano,

Mahea kakou e malu ai ?

3 E hele ana no kakou, He hele hoi ole mai; Ahea la hoi e makaukau $\mathrm{E}$ hele me ka pomaikai?

4 Ua hewa pu ko keia ao; A kii ka Lunakanawai, Mahea na uhane o kakou $\mathrm{E}$ holo maluhia ai?

5 Ke Akua e, hoopono mai I au kauwa hihia nei; He Mohai ke Keiki au, Malaila no ha malu mau.

$2316-8$.

Ka pau koke o ka manawa.

1 AUWE! Ua popo no,

Ko kakou kino nei ;

I keia po, i kela po,

E hoi nui ai.

2 E maule koke ae Ke ola o kakou;

Mohala iki, a ua mae,

A nalo e ua pau.

3 Ke holo kiki nei

$\mathrm{Na}$ hora, me na po; 
Ko kakou wa, he muliwai, E lilo ai kakou.

4 A $i$ lele e na la, Ka hope ke manao, E kiai i kn kaknu wa, A hana naauao.

5 E lawe ia kakou, Maluna o na kai, A pae kakou i kela ao, E pomaikai mau ai.

232.

8.

No ka make ana o ke kino.

1 UA pae pinepine no Ka leo no ko keia ao, "He lepo oe, nolaila mai; Malaila no e hoi ai."

2 Ke mae nei kakou pu a pau, E like maoli me ka lau; Na pua hoi i helelei, Pela kakou e haule nei.

3 A wela iki mai ka la, Mae koke ko kakou mau lau; Nou mai ka ino-aia ka! Ko kakou nani nei, ua pau!

4 Auwe ko kakou make e! Mahea la hoi e malu ai ? 


\section{KUPAPAU.}

Aole anei he ola hou,

He ola loa no kakou?

5 O kau olelo, e Iesu,

He olaio, oia mau;

Ka poe hahai mamuli ou,

He ola loa ko lakou.

Ka make ana, a me ke ala hou ana.

$1 \mathrm{E}$ MAKE ana no anei

Kuu kino make nei?

E hoi nui kuu lala,

I lepo hou anei?

2 E pau no i ka palaho,

A ala hou no nae:

Kuu uhane nei, e komo hou

A hui nani mai.

3 Ua ala hou, e ola mau

Kuu Haku e ola'i;

E kiaiia kuu lehu nei,

A e hookuia mai.

4 Kahiko naniia mai

Ko Iesu mau poliii ;

Maikai na maka, a laelae,

Me kona nani e.

5 Ia Iesu no e lana nei

Ko kakou nei manao, 
$E$ hapai ia ia mau, maanei, $\mathrm{A}$ ia ia ma ke ao.

6 He mele haahaa keia, nou, Hoolohe, e Iesu ;

E mele nani hou makou, Me na clelo mau.

234. 8.

Himeni i ka halawai kupapau.

1 HE lepo no ko keia ao, E hoi ilaila no lakou; He palupalu maoli no, He kino make ko kakou.

2 E nana $i$ neia kupapau, Mamuli pela no kakou; Aole nae i haiia mai Ko kakou la e make ai.

3 He pono hoi, e hoomanao Ia ano make o kakou, E ninau pono ka naau, Auhea la kona makaukau?

4 I keia la, hoomakaukau,

O nele mau, o make e; Paulele koke ia Iesu; Alaila hele pomaikai.

5 E Iesu, e hoomakaukau I na uhane o makou; 


\section{KUPAPAU。}

1awe ola i ke ao,

Me na ohua maikai on.

$$
8--8-6 .
$$

E make ana ko keia ao.

1 E KE Akua mana, e,

E hoomanao ia makou neì,

E make ana no;

Pau koke ko makou mau la;

Aole hiki ke hoopaa

Ka hanu o makou.

\& He mai, kaumaha, kanikau,

Ko makou, a hamama mau

$\mathrm{Na}$ lua kupapau,

Malaila no e iho ai,

$\mathrm{Na}$ mea aloha a maikai,

A me ne hoomalau.

3 Ina nana ma keia ao,

Kaumaha ko makou naak,

Kaumaha wale no;

Aka, ina e wehe ae

Ka buke au i haawi mai

E lana ka manao.

4 Malaila e akaka mai,

He la e ala hou mai ai,

$\mathrm{Na}$ kauwa a Iesu;

Alaila e hoopauia'i

$\mathrm{Na}$ ano o ke kino nes,

A lanalitín mau 
236.

$8-6$.

He wa pokole keia.

$1 \mathrm{E} A O$ mai oe, ke Akua man,

Ke ano o makou,

I ala, a e hoomanao,

E make ana no.

2 He wa pokole ko makou,

Ka wa e noho nei,

He aka, a mae wale no

Ko makou nei maikai.

3 Ke hooikaika nei no nae

Ka poe naaupo,

$E$ ake no e loaa mai

Ka waiwai o ke ao.

4 A loaa mai, pehea la?

Mau anei ia lakou?

Aole hiki ke hoopaa,

Aole emo, pau.

5 Nolaila, e kokua mai,

E Iesu, ia makou,

E kuko, a e imi ae

Ka waiwai oia mau.

$23 \%$

$$
8-6 .
$$

He leo no ka luakupapau.

1 HAMAU kakou, holono e!

He leo kanikau,

He leo mai ka lua mai,

Ka lua kupapau. 
$2 \mathrm{Ke}$ hea mai nei, e nana mai Ka hope o kakou, Kokoke no e iho ai Ia hale pouli mau.

3 Malaila no e nalo ai

$\mathrm{Na}$ kino rani nei,

$\mathrm{Na}$ naaupo, na akamai,

$\mathrm{Na}$ lopa, me na lii.

4 Ka hope keia o kakou;

Palaka nae anei?

E iho aku ana no,

Ia hale pio e?

$5 \mathrm{Nau}$, e Jesu, e paipai mai,

I ko makou naau,

I noonoo i ka hope nei,

A noho makaukan.

Kokoke mai ka make.

1 MAWAENA o na ha la nei,

Ka po a me ke ao,

Maanei, mao, kokoke mai

Ka lua kupapau.

2 Ka uhane kiekie o'u,

E ala, e noonoo,

E hoohaahaa i kou manao,

Nana $i$ ka hope ou.

3 Ka make o ke kino ou, 
Kau ia e nana nei,

Ka make o ka uhane mau,

Kau ia e auwe.

4 Pokole no ka make nei,

Pau emo ole no,

Aka, o kela make e

He make oia mau.

5 E ala, e, mai naaupo,

Mai à ia make mau,

Hahai mamuli o Iesu,

I ola i ke ao.

239.

$$
8-6 .
$$

Iho ana $i$ ka luakupapau.

$1 \mathrm{KE} A$ kua mau, imua ou,

$\mathrm{E}$ ae haahaa makou,

He kino lepo ko makou,

He enuhe make no.

2 Ma kahi no e hele nei,

A hana hoi kakou,

Malaila no ke iho ae,

Ka lua kupapau.

3 Ke ala $e$ hele nei kakou,

Paapu na enemi,

Na mai, me na hihia no,

$\mathrm{Na}$ mea e make ai.

4 E likou nei, e nana mai

Ke kaula makalii, 
O kahi nei e kaulia'i Na mea mau loa'e.

5 Ka make me ke ola nae,

Ke pili mai nei no,

I keia wa e hanu ai,

Palaka nae kakou.

6 Ka Haku, e hookaakaa mai

Na maka o makou,

I nana no, a ike ae,

A hele naauao.

7 Alaila, ina kiiia mai

Na uhane o makou,

Makaukau lakou e pii ae,

E noho me Iesu.

$$
8-8-6 \text {. }
$$

E lanakila ana $\mathrm{i}$ ka make.

$1 \mathrm{E} \mathrm{KUU} \mathrm{uhane} \mathrm{ola} \mathrm{e,}$

E kuu, e kuu ia kino nei,

He eha pono kou;

He make lanakila no,

A waiho keia paio ou,

E pii ae i ke ao.

$2 \mathrm{Ka}$ ! he elele hea nei,

'E kuu kaikaina, hele mai

A launa pomaikai,'

Heaha keia maule o'u? 
Kuu hanu ua kokoke pau, Ka make o'u anei?

3 Ua nalo ae nei keia ao, Ua wehe $a$ mai ka lani no'u,

Memele lea lakou; No'u na eheu e lele ai, Ua aleia ka make, e, Ua pau ka mana ou.

4 I mau eheu i lele ai : Auhea kou mana, e ka po? Ka make, mahea kou? Ua pau, ua lanakila wuu, I ko ka make, ko ka po, Ia Iesu, ke Lii o'u.

2. 3.

1 KA pua i havoli ai, Mohala mai la, a, ua mae; Ua oki koke in! ua pau! Aloha ko kakou naau.

2 Ulono ae la ka naau, E waiho e ka Haku mau, E hookoia nae kou manao, Malaila hoi na kauwa au.

3 Ina e lawe koke ae, Ka whane kau e hormaikai, I ola ia j kela ao, Mohala hou, i nani mau. 
2 Na lesu i hoawi mai

I keia waiwai makamae;

A nana no $i$ lawe hou

Ia pua nani nona no.

5 E na keiki, huli mai,

Ua make e na kamalii,

Nolaila, hoomanao oukou,

A huli koke ia Iesu.

\section{$6-8$}

Ka a make o ke keiki makamae.

1 KAUNAUA no kakou;

Ke waiho make nei

Kekahi keiki a kakou,

He keiki makamae.

2 Ke kanikau kakou,

Uwe waimaka no;

Ua pau ka ike ia ja nei,

Ua pau ka launa pu.

3 E na makua nei,

A me na kamalii,

Nosnoo oukeu, a hoomanao,

Ko kakou ano nei.

1 Na ke Akua mai

Ke keiki make nei,

A nana hoi i lawe ae;

E ae, a hoomailiai:

5 E nalo ana no,

O neia kupapau, 
Aole ike hou ia mas

A pau ae keia a.o.

$6 \mathrm{Na}$ keikĩ neĭ a pau,

We na makna $p u$,

E wiki o hoomakaukar,

No kela da hau.

TRupa pau a ke keikĩ makar.

1 UA eha ko kakow naat,

Ke wwe waimaka nei,

Ke waiho nei he kupaparv

He keiki nakahi.

2 Mahalo ko kakou naau,

la pua makari nei,

Nohala, nono iki pu,

A emo ole mae.

3 Ka nani o te kino nei,

Ka 3 mae ma keia 20 ,

Ma kela a e pud hou as.

He puz nani mau.

4 Nolaila e lana ka manaos.

E ike hou kakou,

Ia keiki nei ma kela ao,

He kino ano hou.

$5 \mathrm{Na}$ kamalii, kakou a pars,

E. nana, a noonoo, 


\section{LA MAHOPE.}

E liuliu iki no,

A make ae kakou.

6 Mai noho a ini ae,

I ko ke kino nei,

E kau ke kuko maluna'e,

Ma kela ao maikai.

\section{La mahope.}

2. "Oí ka lua o ka make."

1 LOHE ia leo mai ka lani mai, Pau ka manawa, aole la hou ac,

E ala hou, e ko na aina a pau,

E hele mai, e ahaolelo no,

Ke Akua wau, ka Lunakanawai,

Ka Haku mana nana e hoopai.

2 Auhea oukou ke aloha waha mai?

Auhea ka hana hoi e pono ai?

Aole loa oluolu wau,

Ia pule hookamani a oukou;

Na mohai a oukou i mohai mai,

Aole loa he mea e malu ai.

3 Ua lohe mai ko oukou pepeiao,

A makemake ole ka naau;

Ua ae ka waha $i$ kuu olelo mau,

Huhu malu la ko oukou naau,

Mamuli o $\mathrm{k} a$ aihue u'ae oukou,

Ka moe kolohe hoi ua launa pu.

$10 *$ 
4 Ca pau ae ner ku kali nu a na's

Pau ka manawa e pemaikai at.

Ke ahi pio ole, o ha po,

Ka lua o ka make aole e pan,

Ka hope ia, ka uku no oukou,

Ka poe hoopenipun mai ia'v.

5 E ala oukou nei ka poe kina,

E kiat hoi, o hiki mai it la;

Hoopololei i ko oukou kekee;

Holo ia lesu, malu koke ai,

O huhu mai ka Lunakanawai,

Make na whane, aole me o ola

245.

$$
8-7 \text {. }
$$

Ka hele hou ana mai o Iesu.

1 AIA hoi ke iho mai la,

Ma ke ao, ke Lï mohai;

Lehuleho na haipule,

Me a Lunakanawai ;

Haleluia! Haleluia!

Haleluia! Amene!

2 Pau na maka i ka ike,

Nana weliweli pu,

Na ku e i ke Keiki,

Uwe, haalulu, me ka $a$;

Mele nui na haipule,

Haleluia ia Iesu.

3 Pau na aina, pau na mauna,

Pau na kai i ke auhee; 


\section{要 MAHOPE。}

Sohe nui na hanauna,

I ka pu e kani nei,

STyele mai, e hoopaia,

Ku imua o ke Lï.

\& Eia mai kela Nesia,

A lakou i pale ae;

Sia mai ka lianakila,

Iesu he Lii pomaikai:

I Ziona, Haleluia?

Als, ca, a haliwai.

5 Oiaio! Non ke aupuni,

Nox, e ke Lii nani $e_{3}$

Hana i akaka nui,

Kou hoopai, he pololer:

Is ka Haku, kii mai oe:

Halelaia! Amenc.

$8-3-6$.

Ka la tropa

1 HE laaole: era mai,

O bo Iehora ta hoopai

Ke he? mai lesu;

Nauweuwe ka honua nei;

Me na hekili nui mä,

Ke kan o ka pu;

2 Eia'e ka Lruakanawai,

E ala pu, a hele maì,

E ko ke ao a pau,

Maalulu mai la na ku e; 
Hoolana no ka poe maikai, Lulumi mai lakou.

3 O ko ka po, o ko ke kai,

Ko lalo o ka lepo nei,

E ku mai, me kakou;

Na lii, na kauwa, na lopa, Hoike like pu, ia la,

I ke Akua mau.

4. Wehe' $a$ na buke o ke ao,

Ua kakauia na hana a pau,

Malaila ka hoopai;

Poho na aia i ka po;

Pii na haipule i ke ao,

Me resu e ola'i.

5 A i nele au e noho nei, Auwe kuu make! knu aje?

Hookahi no Mohai!

Ina o kuu inoa ke kau Ma kana Buke ola mau!

Me e kuu pomaikai!

Ka hope o keia ao.

1 AUWE! He luaole nei ?

O ko ke ao Hopena?

Tia'e ka Lunakanawai,

Ma na ao kaalelewa:

Kaheo ka pur 'E ala hou, 


\section{LA MAHOPE.}

E ko na lua kupapau, A halawai me lesu.'

2 Auwe na kanaka kue

I ko kaknu Mesia!

Mahea lakou e malu ai ?

Me e ka pilikia!

Ua pau na la e pono aí;

Okca keia la hoopai-

Ua hiki mai ka Haku.

3 He nani no ka pomaika

O kana nau haipule!

Pehea kahou ke halawai,

Me ia i ke aouli?

E ala kuu uhane man, Ano e noho makaukau,

E halawai me Iesu.

$$
8-6.6 \text {. }
$$

We'iweli ana ika la hoopal

1 E HIKL in mai ka la, He weliweli e!

E kii ke Lii i na kauwa, Hookolokolo mai.

2 E knu Alii aloha e, Pehea la kuu naau, Ke lohe i kou leo nei, E hele, make mau.

3 Ina ku e ke Akua ia' $\mathrm{a}$, Auwe kuu make e! 
238

\section{H I M E I .}

Ia ahi pio ole no,

E mau loa'i ka uwe!

4 E hai mai, ea! e lesu e,

O kuu inoa ke kau

Ma kou mau lima hoomaikai,

I make ole au.

249.

$$
8-7-4 \text {. }
$$

Ka la mahope.

1 AIA i ka la mahope,

A Iesu e hoi mai ai,

E lohea kona leo,

Kana pu e kani mai,

Lohe pono,

Ko na ilina no a pau.

2 Ko na aina poe make,

Ko na kai mau kupapau,

Mai hikina $a$ komohana,

Mai ka hema a akau,

E hoolohe,

$\mathrm{Ku}$ hou, a lulumi mai.

3 O na ola, me na make,

$\mathrm{Na}$ haipule, na kue,

Na makua, na keiki,

Pau imua o ke Lii,

Lehulehu;

Weliweli ano e!

4 Lohe i ko Iesu leo,

Hele mai, ka poe maikai, 


\section{LA MAHOPE.}

No'u no ko oukeu paulele,

No oukou ke aupuni nei,

Ola loa,

I ka lani, ola mau.

5 E ka poe paulele ole,

Hele oukou i ka po;

Aole i lohe $i$ kuu olelo,

Aohe ola no oukou;

Hele pela!

I ke ahi, make mau.

6 E ka Haku, e hoala

Ia makou e moe ne;,

I kakoo i na puhaka,

Mihi, pule, a kiai;

Aole emo,

Hiki mai ia la hoopai.

$$
6-3 .
$$

Ke ala hou ana.

1 INA e make no,

Ka hanai a Iesu,

Mai $u$ we oukou, e ala hou,

A pii i kela ao.

2 Malaila no e pau

Na ino o keia ao,

Na hewa, luhi, eha, mai,

Aole hiki hou.

3 He nani kela ao,

Ka noho me Iesu, 
Me na uhane, mele pu,

Pomaikai oia mau!

4 Ma ka ilina nae,

E moe ke kino nei,

A ala hou, e loaa mai

Ko luna pomaikai.

\section{Ka Lani.}

(2) 8-6.

- Ko luna kulanakauhale.

1 IERUSALIEMA pomaikai,

Kauhale o ke Lii ;

lini au e komo ae,

Me Iesu kuu Moi.

2 Na papohaku maikai nu,

Na puka momi laa,

$\mathrm{Na}$ alanui gula no,

He nani ke nana.

3 Ko kihapai, ko muliwai,

Me e i kuu manao,

Aole wahi nani e,

Me ia i keia ao.

4 Ina he nani kamahao,

Ko luna aupuni mau ;

Pehea la wau e hoopapau,

A pili $i$ keia ao.

5 Ua hoi kuu Haku i ke ao,

Owau ke ukali ae; 


\section{LANI.}

O kuu mau hoahanau a pau, $\mathrm{E}$ hahai koke mai.

6 Nau, e Iesu e lalau mai,

E hapai ola ia'u,

I kahi mele mau loa's,

$\mathrm{Na}$ anaina mele au.

7 Aloha! e na hoahanaw!

E maluhia nae!

A ma ka lani nani mat,

Fe halawai hou ai.

8 A hela ae la keia ao,

Ka k, me na hoku,

Hoomaka wale ia no,

Ke ola me Lesu.

1 KAUHALE malu, nani e?

Ierusalema hou,

Ahea la pau knu luh nei,

A komo malu wau?

A ike $i$ kou mau pa maikas,

Na pani morni or,

Kou mau laau e malu ai,

Na ala gula mau?

2 He nasi kou Pookcla mata

O Iesu ka Mohai,

Nau, e ke Lii, e lawe at,

Ia wahi e ola'i. 
Na kaula, na haipule hoi, Ke poai ia Iesu,

O ko'u mau hoaaloha nei E hui me lakou.

3 Kauhale o ke Akua e! Ahea la hiki au,

I hou mau aha hoomaikai,

Kou kapu oia mau?

Pehea kuu luhi, kuu makau,

Kuu eha make nei?

A komo wau iloko vu, E pomaikai mau ai.

4 Nalaila mau e mele ai,

Ko lesu poe a pau;

Me e ko lakou pomaikai,

E nani oia mau:

A lau na makahiki ou,

A mano, kini, lau,

A miliona, lehu hou,

E koe ia mele mau.

อง?

$$
8-6 .
$$

Ka Paredino o ke Akua.

1 IERUSALEM $A$ aloha e!

Kauhale pomaikai,

No ko ke $A$ kua mau polii,

E noho loa ai ;

Me e kou pa e nani ai,

Ka papu o ke ao! 


\section{LANI.}

Kon maikai nui, nani nae,

He Paredaiso man

2 Ahea la kuu uhane nei,

Kahiko ia Iesu,

Ma ou laau e malu ai,

A noho malu mau?

Pehea ko lalo ino nei,

Na me' e luhi ai?

Pau na waimaka ia Iesu,

Ma kou mau kumuwai.

3 Ahea la wau e komo ai,

Ia puka momi ou?

A hele me na anela,

Ia ala gula ao?

Ko na aina ola ia Iesu,

Lulumi nui nae,

E ku, kulou, e mele pu,

I kou Alii maikai.

4 Iehova me ka Mohai pu,

Knu luakini mau;

Mahalo loa ia Iesu,

$\mathrm{Na}$ ahamele au:

Ierusalema aloha mau!

Kauhale e ola'i,

No na haipule me Iesu,

E pomaikai mau ai. 
Ke ao mau loa.

1 AIA maluna ma ke ao,

He aina e maha'i,

Malaila no e noho mau,

Ka Iesu poe hanai.

2 Aole po ko laila ao,

He ao mau loa no,

Kolaila poe hauoli mau,

Aole eha hou.

3 He mau kolaila kau maikai,

Na pua kamahao, Aala mai na kihapai,

A uliuli mau.

4 Nawaena nae o kakou nei,

A me ia aina mau,

He kowa e kaawale ai,

Ka make o kakou.

5 Makau kekahi o kakou,

$E$ ae ic kowa nei,

A ku haalulu wiwo no,

Ke hookokoke ae.

6 E pau ko kakou nei makau, E pau ke kuihe,

Nana i $a$ aina maikai mau,

Ka aina inalu e. 


\section{LANI.}

7 E pii, me Mose, ma ka puu, Nana $i$ na kihapai,

Na pua ala nani mau,

Na muliwai maikai.

8 Alaila hee a nalo ae,

Koonei makau a pau,

O ko ka make kowa nei,

Ae oli ae kakou.

$$
\text { 8-6. }
$$

He maha mau loa.

$1 \mathrm{HE}$ wahi malu no oukou,

$\mathrm{Na}$ malihini $u$,

He maha hoi, no na naau,

I eha, luhi, kanikau,

I luna i ke ao.

2 He wahi mau e noho ai,

Ka poe auwana mau,

A luli ae io ia nei,

A kaa ma ko ke ao nei kai;

I luna i ke an.

3 Nana, a lana ka manao,

E o'u mau hoahanau,

Aole emo a e pau,

$\mathrm{Na}$ ino, eha, kanikau,

A komo i ke ao.

4 Malaila no e mohala'i,

Na pua ala man;

Hauoli mau, a pomaikai, 
A lanakila wale ae,

I luna i ke ao.

\section{Hookuu ana.}

256.

$$
\text { 8--7. }
$$

Ka hookuu ana.

1 KO makou Makua nani,

E hookuu aloha mai,

Ho mai hoi i kou Uhane,

E hoomalu, e kiai,

Ia makou na malihini, Ma ka waoakua nei.

2 Maikai wale kau olelo,

A makou i lohe nei,

Ponaikai makou ke hana,

Me ia i kauoha mai;

Nau makou e hoohauoli,

Noho pu a alakai.

3 A pau ae ka makou hana,

O koonei mau halawai,

$\mathrm{Na}$ ko luna poe anela,

E kii mai ia makou nei,

E kaikai iluna lilo,

Me Iesu e noho ai.

$25 \%$

$$
\text { 3-7. }
$$

Ka pau ana o ka halawai.

1 E IEHOVA ke 'Lii mana,

E hoomaikai ia makou, 


\section{HOOKUU ANA.}

I ko makou hoi ana,

I ko makou launa hou,

Ma na hana o ke kino,

Oe pu, kolua mai,

O palaka a poina,

Kau olelo e ola'i.

2 I nana makou iluna,

Hoomanao i kela ao,

I kaawale loa aku,

O na hewa nei a pau;

Hele mau makou imua,

Ma ke ala pololei,

A mai keia waoakua,

$\mathrm{E}$ pii ae $i$ ke ao maikai.

30

8.

Ka hookuu ana.

1 KA Haku e, e hookuu ae,

I kou anaina pule nei,

Me kou hoomaikaiia mai,

Me kou aloha ano e.

2 Na hewa au i ike ai,

Iloko o kou hale nei,

E kala mai, o hoi makou,

Iloko no o kou huhu.

3 He hewa io ko makou,

He lokomaikai wale kou; 
Nana, hoomalu, hoomaemae,

I hoi makou a pomaikai.

250?.

8.

1 E KAU maluna o makou,

Ka hoopomaikaiia ou,

E ka Makua, e Iesu,

Ka Uhane Hemolele pu.

2 I kuikahi pu makou,

Ma ka makou mau hana a pau,

Malaila e hauoli ai,

A ano e ka pomaikai.

26용.

1 NA KEKAHI-KOLU mau,

Ka Makua, me Iesu,

Me ka Uhane, kakou nei,

E hoomalu maoli mai,

Hoomaikai a alakai,

Hoohauoli a kiai,

Launa pu a alu mai,

I mau ae ka pomaikai.

261. 6-8.

E ku a hoomaikaii ke Akua.

1 E KU a hoomaikai,

Ka Haku o kakou,

Hoonani mau na leo nei,

A hui na naau. 
Hanai man mai me ka mane, Kai ma kahi e ola'i.

2 Hoohauoli mai $i$ na houpo. I lea ai na nule nou; Aia hik sou aupuni, Nou e hapai ai makou, Ne na Serapima ou.

3 Mele pu, Malchia, Amene, Naleluia, Fali nou, Malelusa,

Ia Logen Resia, Ke Akua makai.

4 Nole man, Malkida,

Dia mau, Maleluia,

Ia lesu, Halchia,

$$
\text { I ke Lii Violiai, }
$$

5 Malelua, Amene; Ancre; Arrenc.
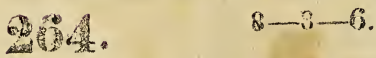

I IKA Makua nani 0

Nana i hana kakou nei,

Ka hommalia mau no;

I ka Mosia e ola't,

A rie ka Uhane hoomacnae,

Hoonazi mau kakou. 


\section{HกOKUU ANA.}

$8-8$.

HIILANI $i$ ka Makua mau,

Ke Keiki Lngou kamahao,

Ka Uhane Hemolele pu,

Ke Akua Kahi-kolu no,

Iehova ke Lii o kakou;

Hoomaikai mau ia, no Iesu.

26.

$7-6$.

HOOMAIKAI $i$ ka Makua,

Ke Aivua o kakou;

Hapai i ke Keiki,

- I kona like no;

Hoonani i ka'Uhane,

Ke Akua hoomaemae;

Hookahi Haku nani,

Ke Akua e o'a'i.

HAPAI, i ke ao, i ka po,

I ko ke ao Haku maikai:

Hapai $i$ ka Makua kakou,

Ke Keiki Mohai e ola“i;

Hapai i ka Uhane maikai;

$\mathrm{Ke} A$ kua honkahi lakou;

He kupaianaha no nae,

Hoomana na mea a pau. 


\section{8.}

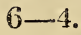

KU MAI, e hapai pu,

I ka Makua mau;

Hoonani ia Iesu,

Ke Akua o ke an,

K $a$ Uhane mau, ke Akua no;

Hiilani pu na ao a pau.

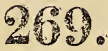

$$
6-6-8 \text {. }
$$

E MOE na kanaka,

Kuiou na anela,

Hoolea i ka Mikua mau

Ke Keiki ka Logou,

$\mathrm{K}$ a Uhane nani no,

Hoomaikai oia mau kakou.

\section{0.}

E HOONANHA ke Akua,

Ma na lani kiekie loa;

He malu hoi ma ka honua;

He aloha no i kanaka.

\section{篦1.}

I E HOOKANI olioli in Iehova, e na aina a pau:

E malama ia Lehova, we ka hauoli,

E hele mai imua o kona alo, me ke oli ana;

I ike oukou, o Iehova, oia no ke Akua : 
2 Nana kakou i hana, aole na kakou kakou;

0 kona kanaka hoi kakou, ns hipa hoi o kona kula ; E komo i na jpuka ona, me ka mohai aku i ke aloha : Hoko hoi o kona pahale, me ka hoomaikai.

3 E aloha aku ia ia, e mahalo aku hoi i kona inoa ; No ka mea, he maikai no lehova;

E mau loa ana knna aloha,

E nau lou ana hoi koni oiaio, i na hanauna a pau:

272

8.

HOONANI $i$ ka Makua mau,

Ke Keiki me ka Uhane no,

Ke Akua mau-Hoomaikai pu,

Ko keia ao, ho kela ao.

$2 \%$

$8-6$.

HAPAI $i$ ke Akua e ola'i,

I ka Makua mau,

Ke Keiki me ka Uhane hoi,

Mahalo mau kakou.

HAPAI e na anela,

I ka Makua mau,

Ka Logou, me ka Uhane laa,

Hoolea pu keia ao. 


\section{PAPA LALANI MUA.}

Aoao. Himeni.

Aia i ka la mahnpe, - $\quad$ - $233 \quad 249$

Aia hoi ke iho mai la, - $-2: 34 \quad 245$

Aia hoi! ke ku mai nei, - $83 \quad 81$

Aia hoi! ko lesu leo, - - 4844

Aia ko ke ao nei ola, _ $\quad 4945$

Aia maluna ma ke ao, - $\quad 8987$

Aia maluna ma ke so, - $-244 \quad 254$

Auhea ka pono mau, - - 147

Auhea oukou, na honmalau, 8178

Auhea oukou na keiki hou, $163 \quad 168$

Auwe, e kuu Akua e! - - $42 \quad 38$

Ausve! hohonu e la po! - $90 \quad 88$

Auwe! he lua ole nei! - $-236 \quad 247$

Auwe ka poe i honpanee, $73 \quad 71$

Auwe! ke noho nei no wau, 9897

Auwe! ua kahe wale ae, - 172 179

Auwe! ua hewa maoli no, $105 \quad 105$

Auwe! ua popo no, - - $221 \quad 231$

Ahonui wale Iesu, - $\quad$ - 4440

A huli mai na boomalau, - $155 \quad 159$

\$ kau ko Iesu kanawai, - $104 \quad 103$

Ala, ala, e nana, - - -219229

Ala'e oukou, _ _ _ + - $142 \quad 143$

Ala'e kakou, - - - - 1:37 138

Aloha, e na hoahanau, - $-126 \quad 127$ 


\section{5}

A020. Hime i.

Aloha ino o lakou, - - 65

6is!

Aloha oe, kuu Haku, e, - 98

96

Aloha kakou nei, - - - 199

208

Aloha ko na matra, - - - 182

189

Aloha nu ko lesn, . . - 37

$2: 3$

Alno mai, kus Hahue, - 102

1 \& 1

A manpono lea is'u, - .. -130

182

A pay na la, a whi mai, - 134

135

E. ao mai oe, ke Akua mau, $226^{\circ}$

286

E ala, e na hoahanau, - - 157

161

Wala, o na mokn e,

$-196$

205

E ala knu uhane nei.. - - 117

118

Ji ala havon rei a pau, - - 179

$180^{\circ}$

Tohia kcia wah! - . - 176

183

Fia mai ke mele hor, - - 36

31

Flehova he Akua, - . - 114

115

E lehova ke 'Lii mant,

E lesu ha makai, - - -

$-246$

257

22

F Tesu ka mohai no's, - - 130

131

)

$-207$

217

L ihn, me ka mana ou, - - 59

55

Fi oli atu hakou nei, - -18

11

$-112$

$11 \%$

Fir, na koa o lesu; - - - 118

119

Whele olioli mai. - - - 187

195

E hele no wan in Iesu, - -108

108

Fi hakanu i ka Mni, - - 113

113

E hiki io mai ka la, - - -237

248 


\section{$25 \%$}

Aonn. Himent.

E hookani olioli aku ia Te- $252 \quad 271$

E hoomaka i ke kani, - -45

41

E honnaniia ke Akua, - $-25 \% \quad 270$

E huli, e kanaka auwana nei, $7 \%$

E huli mai, e huli mai, - $-88 \quad 86$

E kai ne, e lehova, - -115116

E ka Uhane Hemol le, - - $61 \quad 58$

E ka Haku mana e, - - $-200 \quad 209$

E ka Makua e, he nani kou, 1:3 5

E ka Puuhonua man, - $\quad-136 \quad 137$.

E kau maluna o makou, - $248 \quad 259$

E ke Akua ke Lii mau, - 164

E ke Akua mana. $e, \quad-\quad-2.5$

E ke Akua mana man. - - 22

E ke Akua mana man, - -206

E ke Akua pono mau, - - $166^{\circ}$

E ke keiki hipa e, - - - 171

E ke 'Lii o na ao a pau, - 193

F.ke 'Lii mana mau, - $\quad 24$

I ko ke ao, hoolohe mai, - 78

E ko ke ao, haunli pu, - - 5:3

F koho no ka poe aia, - -74

E ko Tehova lima e, - - -201

E ko Tehova poe kauwa, - 204

S ko makou Akua e, - $\quad 189$

169

$2: 35$

15

216

172

178

207

18

35

49

72

:10

$2 ! 4$

E ko makou Makua e, - - 96

1.97

S kokoke io ana, - _ $\quad-20 \cdot 2$

F. kuu Akua maikai, e, - - 97

94

211

E kuu Uhane, e noonoo, - 217

95

227 
Aoro. Himeni.

F kuu uhane ola e, - $\quad-229 \quad 240$

E ku a hoomaikai, - - 248261

E ku e hou anei, - _ - $\quad 9998$

E kuhikuhi mai, - $\quad$ - $\quad 3226$

E lawe aku ana, - $\quad$ - 218228

E lawe nui mai, - $\quad$ - $\quad$ - 128129

Eimake and no anei ? - - 233 233

E mele oli, e, - - - - $5 \%$

E mele akn kakou nei, - - $22 \quad 222$

E mele kakru nei, - - -213

E mihi, wahi a lesu, - - -81

203

79

E moe na kanaka, - $-\quad-252$

269

F na haipule nei.; - - $\quad$ - 56 52

E na haipule nei, - $\quad$ - -104109

F na hoshasau aloha, $-\quad-119120$

Ena kiai, e hai mai, - - -197206

E na kiai o lesu, - - - -192200

E na ohua pomaikai $\quad-\quad-159 \quad 163$

E na milihiıi nei, - - - $-132 \cdot 133$

Tehova e aloha mai, - $-\quad-100 \quad 99$

Iehova ke Akia mau, - $-146 \quad 148$

lehova ke 'Liii mau, - - - 2j 19

Iehova uo lie Akua mau, - 120 121

Iehova no kuu Kahu mau, $17 \quad 10$

Ierusilema aloha e! - - $-242 \quad 253$

Ierusalema pomaikai, - $-240 \quad 2,31$

Iesu no ka Mane mau, - $-169 \quad 175$

I kela wa i noho ai, - . $-181 \quad 188$ 


\section{8}

Ansn. llimeni.

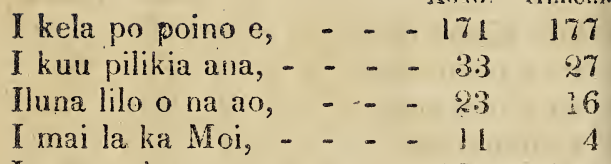

Ina e make no, _ $\quad$ - -239250

Ina me kiu Akua mau, - $124 \quad 125$

O lesu ka inoa maikai, - - $30 \quad 24$

O Iesu Kristo ke 'Lii mau, $34 \quad 28$

O keia wa. he wa maikai, - 156160

Ololo ko ka aia na"u, - - 93991

Oli aku ia Iehova, - $\quad$ - $19 \quad 12$

Owai ka make'wai, - - $6 \% 64$

Owai la i hoolohe mai, - -28 21

Owai la ka mea e hoike mai, 185. 193

Ua alohaia mai kakou, - $\quad 31$

Ta eha ko kakou naau, - - 332

Ua ino paapu no, - - -101

Ua olioli kuu naau; - $-\quad 123$

U'oki, ea! e huli mai, - - 7 !

Ua hala keia la, _ _ - 209

Ua hiki mai ka la maikai, - 144

Ua hoounaia mai, - _ _ $\quad$ - 5

Ta loknmaikai no Iesu, - - 167

Ua makaukau ka aina lou, 183

Ua pae pinepine no, - - -222

25

243

100

124

69

$2 \div 9$

146

20

$17: 3$

Ua wehe inai ke alaula hou, 208

100

$2: 3 \times 2$

Uhane laa kuv mai, - _ - $6 \%$

218

Uhane mau boolono mai, - 58

5)?

54 


\section{9}

Aoxo. Himeni.

Haalele e ka hawawa, - - 75

73

Hauoli, e Ziona, e, - - 122

123

Hauoli i ka la maikai ; - 141

142

Hauoli pu, Iesu ke 'Lii, - 154

157

Hauoli wale au; - - - 149

1.52

Hamau! ke kani nei ka pu, 80

77

Hamau kakou, holono e! - 226

2.37

Hapai i ke ao, i ka po, - - 251

267

Hapai i ke Akua e ola'i, - 253

2.3

Hapai e na anela. - - -253

274

He Akua Hemolele, - - - 10

3

He Akua pono no; - _ -66

63

Heaha la kakoui - - - 214

221

He ahaaina nei, - - - 175

He abonui wale mai, - $\quad 35$

182

30

He aloha ko Iehova, - 15

He olioli e; - - - - - 152

He hana e hauoli ai, - - 143

He hanohano mau, - - 40

He hoailona wale no, --162

He kulanahale ku paa, - $\quad 38$

Hele mai i ka Mesia, _ - 77

Hele mai ka poe luhi, - - 67

He lepo no ko keia ao, - - 224

He 'Lii hoano mail, - - - 55

He luaole! eia mai, - - - 235

He luaole hoi, - - - - 186

He lokomaikai ko Iesu, - - 165

8

155

144

36

166

34

74

65

234

51

246

194

170

He nani no ko'u pomaikai, 107

107 


\section{0}

A

He nani kona mau wawae, $144 \quad 145$

He nani no na kapuwai, $\quad-195203$

He pono no kakou maanei, $148 \quad 151$

He wahi eehia nei, - - $\quad 146 \quad 149$

He wahi malu no oukou, - $245 \quad 255$

Hiilani i ka Makua mau, - 251265

Hoi mai, e kuu uhane, - - $121 \quad 122$

Hoano ko Ziona pun, - - 5248

Hoomaikai nui ia Iesu, - $-37 \quad 33$

Honmaikai i ka Makua, $-251 \quad 266$

Hoonani, e honmani mau, - $23 \quad 17$

Hoonani na pokii, - - 135136

Iloonani e na aila nei, - -211.221

Hoonani i ka Makua mau, $253 \quad 272$

Hookani i ke 'Lii maikai, - 5450

Hoku loa, a:ıo e, - - - $140 \quad 141$

Hoolohe, e ka poe mai, - 154158

Horlohe, e ka poe kue, - $87 \quad 85$

Iloolea na kanaka a pau, - $181 \quad 191$

Hoolea aku ko ke ao, - $-20 \quad 13$

Holo maha ole io, - $-216 \quad 226$

Hosana i ka Haku mau, - 8684

Wa Uhane Hemolele mau, - $168 \quad 174$

Ka Uhane mau, hoopuka mai, $63 \quad 60$

Ka Uhane mau, ua hiki mai, $151 \quad 154$

Kaumaha wau m:ıanei, - - 111, 111

Ka ua i haule mai, - - -6461

Ka Haku e, e iho mai, - $-138 \quad 139$ 


\section{\$1:}

Ano Himenis.

Ka Haku e, e Hho mai, - - 180187 Ka Haku e, e nana mai, - 105104 Ka Haku e, e hookuu ae, - 247258 Ka Haku e, palaka wau; - 8482 Ka Makua nani e, - - - 250 264 Kaumaha no kakou, - - - $231 \quad 242$ Kauhale malu, nani e! - - $241 \quad 45 \%$ Kakoo, e ke Lii mónar e, - 1932 201 Ka poe nani oke an, - $\quad 30 \quad 23$ Ka pua i hatoli ai, - - -230 241 Kei ka mana o Iehova, - $-10 \quad 2$ Ke Alku e, e hana hou, - $106 \quad 106$ Ke Akwa mau, imua ou, $\quad-228 \quad 239$ Ke Akua lokomaikai man, $210 \quad 220$ Ke Akua, ko'u Ali maikai, $161 \quad 165$ Ke Akua o ke ao, - $\quad$ - 146 Ke Akua nona e ola'i, - - 16; 9 Ke hoomaikai abu nei ma- 249 263 Ke holo nui nei lakou, - - $34 \quad 29$ Ke kau nei no maluna o'u, 82 Ke lawe ia nei, - - - 166 $\mathrm{K}$ ə ola! o ke ola man! - - 43 Ke ola no ka mea maikai, - 63 Ke nana akı nei, - - - 129 Ko keia an, he lepo no, - $-2: 30$ Ko makou Alii, - - Ko makou Makua nani, - $246 \quad 256$ Knu nani, e ke Lii, - - - 125126 Ko makou Haku mau, - - $60 \quad 57$ 


\section{2}

Aохо. Himeni

Ko makou Kahuna mana, - 46

42

Komo, Iesu, i kou mala, - 113

114

Kuu ia makou, e ka Haku, 249

263

Kuu uhane e kiai, - - 133

134

Kuu Haku, e aloha mai, - 103

102

Ku mai, e hapai pu, - - -252

Kupanaha ke aloha, - - 47

268

43

Laula ke ala e iho ai, _ $\quad$ - 69

Lohe'a ka leo'mai Sinai, - 70

67

Lohe ia leo mai ha lani mai, 233

68

244

Maanei kakou e homanao, 173

Mai ke kea o Iesu, - - - 174

Mai ka lani, iho mai la, - 41 ,

180

181

Ma keia papa sina au, - -177

37

184

Ma ka wai o Inredane, - -158

162

Ma ka puu o Kalevari, - -170

Ma ko Lehova alo mau, - - 9

176

Ma ko lesu inoa maikai, - 60

56

Ma ko Babela muliwai, - - 127

128

Ma na aina pouli wale, - -203

212

Ma na mauna pouli wale, - 188

Mawaena o na hana nei, - 227

196

238

Mele i ka Iubile, - - - - 184

192

Mele pono ia Iesu, - - 39

35

Na anela, na krnaka, - $\quad 162$

167

Nana kakou, mai o a o, - 153

156

Nani ka Iesu olelo, - - 92

90 


\section{3}

Aoro. Hmeni.

Nani lna kou ka hale, - $-145 \quad 147$

Nani keia Sabati, - - $-147 \quad 150$

Nani na wawae o lakou, - $194 \quad 202$

Wa elele a lesu, - $\quad$ - $-205 \quad 215$

Na hauwa a ke Aliua, - -190198

Na ke aha e hoohuli, - $-203 \quad 213$

Na kekahi-kolu nau, - $-248 \quad 260$

Na ko kakou Akua mau, - $178 \quad 185$

Na miliona nui no, - - -195204

Na rakahiki o kakou, - $-215 \quad 225$

Min Tesu ka homna, - - - 51 47

No Tesu ke aloha mau, $-\quad-116 \quad 117$

Poino no na hoomalau, - - 7976

Poino nui ko ka po, - - 9189

Pokole keja wa, - - _ 8583

Pomaliai ke kanaka ao, - 9499

Pomaikai waje kn ke ao! - $191 \quad 199$

Pomaikai wale o lakou, - -110110

Pomaikai au i keia la, - $-160 \quad 164$

Pono mau ka la maikai, - 5046

Ponlikeia an, - - - - 139140

Puhi i ka pu akou, - - $-150 \quad 153$

Pule pono ia Lehova, - $\quad 21 \quad 14$ 


\section{HE PAPA ANO HIMENI.}

No ke Akua. No lesu. .

Uhane Hemolele. Hooweliweli. Mihi. . Hoahanau. . Sabnti. . . . Pule Hoomate. . . 151-15\% aoao. Bapetizo ana. . $\because 158^{\circ}-168$ acan. Ahavina a ka Haku. 169-175 aoao. Mare. . . . . 178-181 aoan. Malama Hout. . . . 188-205 anan. Hoolna ana i lea Lualini. 206. 206 ao Krthahika. . . . 208 anao. Ahichi. . . ¿. 209 aoa. Hoomaikai ara. . . 210-213 aoaa. Hope o ka Makahili. 214 aoao. Makahiki Hou. . . 215-219 anao. Кирараи. . . . . 220-232 аеао. La Miphope. . . . 23:3-239 aоao. Ka Lani. . . . . $240-245$ амдо. Hookuu ana . . .246-253 aoao. 





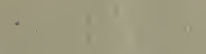

I. 
s.

18) 3 (2)

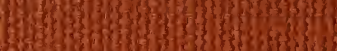

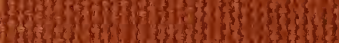

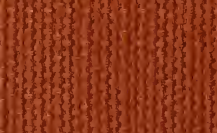

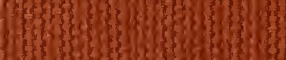

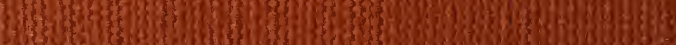

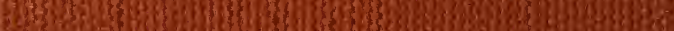

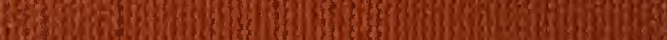

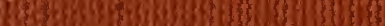

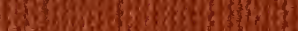

8.

2.

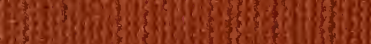

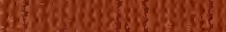

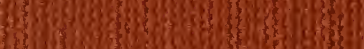

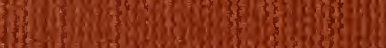

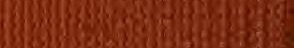

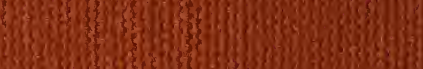

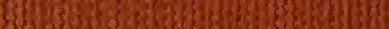

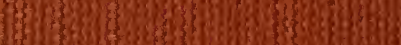

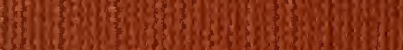

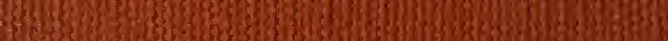

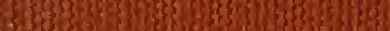
2.

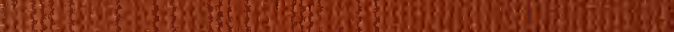

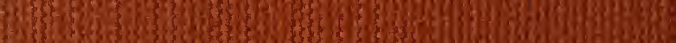

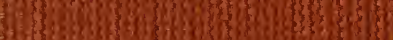
(x)

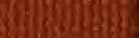

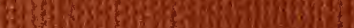

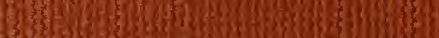

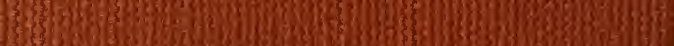

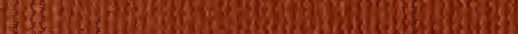

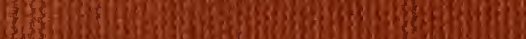
is

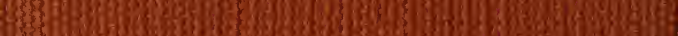

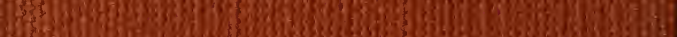

